\title{
Positive Scalar Curvature on Spin Pseudomanifolds: the Fundamental Group and Secondary Invariants
}

\author{
Boris BOTVINNIK ${ }^{\mathrm{a}}$, Paolo PIAZZA ${ }^{\mathrm{b}}$ and Jonathan ROSENBERG ${ }^{\mathrm{c}}$
}

a) Department of Mathematics, University of Oregon, Eugene OR 97403-1222, USA

E-mail: botvinn@uoregon.edu

URL: http://pages .uoregon.edu/botvinn/

b) Dipartimento di Matematica "Guido Castelnuovo", Sapienza Università di Roma, Piazzale Aldo Moro 5, 00185 Roma, Italy

E-mail: piazza@mat.uniroma1.it

URL: http://www1.mat.uniroma1.it/people/piazza/

c) Department of Mathematics, University of Maryland, College Park, MD 20742-4015, USA

E-mail: jmr@umd.edu

URL: http://www2.math.umd.edu/ jmr/

Received May 26, 2020, in final form June 08, 2021; Published online June 24, 2021

https://doi.org/10.3842/SIGMA.2021.062

\begin{abstract}
In this paper we continue the study of positive scalar curvature (psc) metrics on a depth-1 Thom-Mather stratified space $M_{\Sigma}$ with singular stratum $\beta M$ (a closed manifold of positive codimension) and associated link equal to $L$, a smooth compact manifold. We briefly call such spaces manifolds with $L$-fibered singularities. Under suitable spin assumptions we give necessary index-theoretic conditions for the existence of wedge metrics of positive scalar curvature. Assuming in addition that $L$ is a simply connected homogeneous space of positive scalar curvature, $L=G / H$, with the semisimple compact Lie group $G$ acting transitively on $L$ by isometries, we investigate when these necessary conditions are also sufficient. Our main result is that our conditions are indeed sufficient for large classes of examples, even when $M_{\Sigma}$ and $\beta M$ are not simply connected. We also investigate the space of such psc metrics and show that it often splits into many cobordism classes.
\end{abstract}

Key words: positive scalar curvature; pseudomanifold; singularity; bordism; transfer; $K$ theory; index; rho-invariant

2020 Mathematics Subject Classification: 53C21; 58J22; 53C27; 19L41; 55N22; 58J28

\section{Introduction}

This paper continues a program begun in [10, 15], and our previous paper [14] (hereafter called Part I), to understand obstructions to positive scalar curvature on manifolds with fibered singularities, for metrics that are well adapted to the singularity structure.

As in [14], the stratified spaces (or singular manifolds) $M_{\Sigma}$ that we study are Thom-Mather pseudomanifolds of depth one, where we take the two strata to be spin. Topologically, $M_{\Sigma}$ is homeomorphic to a quotient space of a compact smooth manifold $M$ with boundary $\partial M$. The manifold $M$ is called the resolution of $M_{\Sigma}$, and the quotient map $M \rightarrow M_{\Sigma}$ is the identity on the interior $\stackrel{\circ}{M}$ of $M$, and on $\partial M$, collapses the fibers of a fiber bundle $\phi: \partial M \rightarrow \beta M$, with fibers all diffeomorphic to a fixed manifold $L$, called the link of the singularity, and with base $\beta M$ sometimes called the Bockstein of $M$ (by analogy with other cases in topology). While we do

This paper is a contribution to the Special Issue on Scalar and Ricci Curvature in honor of Misha Gromov on his 75th Birthday. The full collection is available at https://www.emis.de/journals/SIGMA/Gromov.html 
treat the general case for some of our results, we shall eventually adopt the main geometric assumptions from [14], namely that the bundle $\phi$ comes from a principal $G$-bundle $p: P \rightarrow \beta M$, for some connected semisimple compact Lie group $G$ that acts transitively on $L$ by isometries for some fixed metric $g_{L}$, and thus $\partial M=P \times_{G} L$. The transitivity of the action of $G$ on $L$ means that $L=G / H$ is a homogeneous space which comes with a metric $g_{L}$ with constant positive scalar curvature, which we normalize to be the same as that of a sphere of the dimension $\ell=\operatorname{dim} L$.

Given a Lie group $G$ and a link $L=G / H$ as above, we say that a fiber bundle $E \rightarrow B$ with a fiber $L$ is a geometric $(L, G)$-bundle if its structure group is $G$, where $G$ acts on $L$ by isometries of the metric $g_{L}$.

An adapted metric $g$ on $M_{\Sigma}$ will be defined by the following data: a Riemannian metric $g_{M}$ on $M$, which is a product metric $\mathrm{d} t^{2}+g_{\partial M}$ in a small collar neighborhood $\partial M \times[0, \varepsilon)$ of the boundary $\partial M$, a connection $\nabla^{p}$ on the principal $G$-bundle $p$, and a Riemannian metric $g_{\beta M}$ on $\beta M$. We require the metrics $g_{L}, g_{\partial M}$ and $g_{\beta M}$ to be compatible in the sense that the bundle projection $\phi:\left(\partial M, g_{\partial M}\right) \rightarrow\left(\beta M, g_{\beta M}\right)$ is a Riemannian submersion with fibers $\left(L, g_{L}\right)$. Furthermore, since the structure group $G$ of the bundle $\phi: \partial M \rightarrow \beta M$ acts by isometries of the metric $g_{L}$, we can make the special metric $g_{L}$ on the fibers orthogonal to the metric $g_{\beta M}$ lifted up to the horizontal spaces for the connection $\nabla^{p}$. Then $M_{\Sigma}$ is the result of gluing together the Riemannian manifold $M$ and a tubular neighborhood $N$ of $\beta M$ along their common boundary $\partial M$. The complement of $\beta M$ in $N$ will look like a fiber bundle over $\beta M$ whose fibers are open cones $(0, R) \times L$, where $R$ is the radius of the cones. We require these fibers to be actual metric cones with the metric $\mathrm{d} r^{2}+r^{2} g_{L}$ (which is actually a warped product metric on the product of $L$ with the interval $(0, R))$, transitioning smoothly near $r=R$ to a product metric $\mathrm{d} r^{2}+C g_{L}$ for a suitable positive constant $C$. In this paper, we allow for $M$ and $\beta M$ to have non-trivial fundamental groups; the simply-connected case was considered in Part I [14]. Throughout this paper we assume that the link $L=G / H$ is a simply connected homogeneous space, where $G$ is a compact semisimple Lie group acting on $L$ by isometries of the metric $g_{L}$, and where

$$
\operatorname{scal}_{g_{L}}=\operatorname{scal}_{S^{\ell}}=\ell(\ell-1)
$$

This normalization makes the cone on $L$ scalar-flat with respect to the metric $\mathrm{d} r^{2}+r^{2} g_{L}$. This will be important for our eventual existence results since this will guarantee that if $g_{\beta M}$ has positive scalar curvature, then we can make the scalar curvature uniformly positive on the tubular neighborhood of $\beta M$. In this setting, it is easy to see (since $L$ is simply connected) that $\pi_{1}(\partial M)=\pi_{1}(\beta M)$, and then Van Kampen implies that $\pi_{1}\left(M_{\Sigma}\right)=\pi_{1}(M)$.

Theorem 1.1 (obstruction theorem). Let $M_{\Sigma}$ be an n-dimensional compact pseudomanifold with resolution $M$, a spin manifold with boundary $\partial M$. Assume the following:

(1) $M$ is a spin manifold with boundary $\partial M$ fibered over a connected spin manifold $\beta M$,

(2) the fiber bundle $\phi: \partial M \rightarrow \beta M$ is a geometric $(L, G)$-bundle.

Let $\Gamma=\pi_{1}(M), \Gamma_{\beta}=\pi_{1}(\beta M)$. Assume $g$ is an adapted Riemannian metric on $M_{\Sigma}$ and let $g_{M}$ be the restriction of $g$ to $M$. Then there are two "alpha invariants", generalized indices of Clifford algebra-linear Dirac operators,

$$
\alpha_{\text {cyl }}^{\Gamma}\left(M, g_{M}\right) \in K O_{n}\left(C_{r, \mathbb{R}}^{*}(\Gamma)\right) \quad \text { and } \quad \alpha^{\Gamma_{\beta}}(\beta M) \in K O_{n}\left(C_{r, \mathbb{R}}^{*}\left(\Gamma_{\beta}\right)\right) \text {. }
$$

The indices $\alpha_{\text {cyl }}^{\Gamma}\left(M, g_{M}\right)$ and $\alpha^{\Gamma_{\beta}}(\beta M)$ do not depend on a choice of adapted metric $g$, and they both vanish if there exists an adapted psc metric on $M_{\Sigma}$. 
Remark 1.2. In our case we fix the metric $g_{L}$ on $L$ and the connection on the bundle $\phi: \partial M \rightarrow \beta M$ coming from a connection $\nabla^{p}$ on the principal bundle $p: P \rightarrow \beta M$. (This is harmless since the space of such connections is contractible.) Then an adapted wedge metric $g$ on $M_{\Sigma}$ is determined (up to contractible choices) by a metric $g_{\beta M}$ (which then determines the metric $g_{\partial M}$ ) and by an extension $g$ of $g_{\partial M}$ to $M$. Thus the space of adapted wedge metrics on $M_{\Sigma}$ is contractible, and from the analytic properties of Dirac operators we then obtain that the cylindrical $\alpha$-class $\alpha_{\text {cyl }}\left(M, g_{M}\right)$ is independent of $g$ (once the metric $g_{\beta M}$ has been fixed). Hereafter, we will omit the metric $g$ from the notation. Notice that there is also a wedge $\alpha$-class $\alpha_{w}^{\Gamma}\left(M_{\Sigma}, g\right)$ defined by considering the Dirac operator on the regular part of the pseudomanifold $M_{\Sigma}$. This is also independent of $g$ for $(L, G)$-fibered pseudomanifolds. The two classes $\alpha_{\text {cyl }}^{\Gamma}(M)$ and $\alpha_{w}^{\Gamma}\left(M_{\Sigma}\right)$ are equal if the metric $g_{\beta M}$ is psc. However, in more general situations, the wedge $\alpha$-class $\alpha_{w}^{\Gamma}\left(M_{\Sigma}, g\right)$ and the cylindrical $\alpha$-class $\alpha_{\text {cyl }}^{\Gamma}\left(M, g_{M}\right)$ both depend on the choice of metric $g$ and they are in general different. We shall make all this very precise in the next section.

Now we are ready for the existence result.

Theorem 1.3 (existence theorem). Let $M_{\Sigma}$ be an n-dimensional compact pseudomanifold with resolution $M$, a spin manifold with boundary $\partial M$. Assume the following:

(1) $M$ is a spin manifold with boundary $\partial M$ fibered over a connected spin manifold $\beta M$,

(2) the fiber bundle $\phi: \partial M \rightarrow \beta M$ is a geometric $(L, G)$-bundle.

Let $\Gamma=\pi_{1}(M), \Gamma_{\beta}=\pi_{1}(\beta M)$. Furthermore, assume $n \geq \ell+6$, where $\ell=\operatorname{dim} L$, and that one of the following condition holds:

(i) either $L$ is the boundary of a spin $G$-manifold $\bar{L}$ equipped with a $G$-invariant psc metric $g_{\bar{L}}$, which is a product near the boundary and satisfies $\left.g_{\bar{L}}\right|_{L}=g_{L}$,

(ii) or the embedding $\partial M \rightarrow M$ induces an isomorphism on $\pi_{1}$, and moreover, $\partial M=\beta M \times L$, where $L$ is an even quaternionic projective space, and $\Omega_{*}^{\text {spin }}(B \Gamma)$ is free as an $\Omega_{*}^{\text {spin }}$-module.

Then, provided the Gromov-Lawson-Rosenberg conjecture holds for the groups $\Gamma$ and $\Gamma_{\beta}$, the vanishing of the invariants

$$
\alpha_{\text {cyl }}^{\Gamma}\left(M_{\Sigma}\right) \in K O_{n}\left(C_{r, \mathbb{R}}^{*}(\Gamma)\right) \quad \text { and } \quad \alpha^{\Gamma_{\beta}}(\beta M) \in K O_{n}\left(C_{r, \mathbb{R}}^{*}\left(\Gamma_{\beta}\right)\right)
$$

implies that $M_{\Sigma}$ admits an adapted psc metric.

Remark 1.4. We notice that the condition $(i)$ holds when $L$ is a sphere, an odd complex projective space, or when $L=G$.

In the last part of this paper, Section 6 , we begin to analyze the homotopy type of the space $\mathcal{R}_{w}^{+}\left(M_{\Sigma}\right)$ of adapted metrics of positive scalar curvature on $M_{\Sigma}$, in the case where this space is non-empty. One of the key results is the following.

Theorem 1.5. Let $M_{\Sigma}$ be an $(L, G)$-fibered spin pseudomanifold, and assume that $M_{\Sigma}$ admits an adapted psc metric. Fix a connection $\nabla^{p}$ on the associated principal $G$-bundle over $\beta M$. Let $\operatorname{res}_{\Sigma}: \mathcal{R}_{w}^{+}\left(M_{\Sigma}\right) \rightarrow \mathcal{R}^{+}(\beta M)$ be the forgetful map sending a psc metric $g$ on $M_{\Sigma}$, interpreted as a pair $\left(g_{M}, g_{\beta M}\right)$, to the metric $g_{\beta M}$ on $\beta M$. Then $\operatorname{res}_{\Sigma}$ is surjective onto $\mathcal{R}^{+}(\beta M)$, and it has a (non-canonical) section. In particular, there is a split injection of the homotopy groups of $\mathcal{R}^{+}(\beta M)$ into those of $\mathcal{R}_{w}^{+}\left(M_{\Sigma}\right)$. 
We use this result to detect non-trivial homotopy groups of the space $\mathcal{R}_{w}^{+}\left(M_{\Sigma}\right)$. Namely, we let $M_{\Sigma}=M \cup-N(\beta M)$, as before. Once we fix a base point, a metric $g_{0} \in \mathcal{R}_{w}^{+}\left(M_{\Sigma}\right)$, it determines corresponding metrics $g_{\beta M, 0} \in \mathcal{R}^{+}(\beta M)$ and $g_{M, 0} \in \mathcal{R}^{+}(M)_{g_{\partial M, 0}}$ (where $g_{\partial M, 0}$ is given by the metric $g_{\beta M, 0}$ and $\left.g_{L}\right)$. Then we have index-difference homomorphisms:

$$
\operatorname{inddiff}_{g_{\beta M, 0}}: \pi_{q}\left(\mathcal{R}^{+}(\beta M)\right) \rightarrow K O_{q+n-\ell}
$$

and

$$
\operatorname{inddiff}_{g_{\partial M, 0}:} \pi_{q}\left(\mathcal{R}^{+}(M)_{g_{\partial M, 0}}\right) \rightarrow K O_{q+n+1},
$$

where $K O_{k}$ is the $k$-th coefficient group for real $K$-theory (equal to $\mathbb{Z}$ for $k$ a multiple of 4 and $\mathbb{Z} / 2$ for $k \equiv 1,2 \bmod 8$ ); see Section 6 and [11, 24]. According to Theorem 1.5, we can choose a splitting

$$
\pi_{q}\left(\mathcal{R}_{w}^{+}\left(M_{\Sigma}\right)\right)=\pi_{q}\left(\mathcal{R}^{+}(M)_{g_{\partial M}}\right) \oplus \pi_{q}\left(\mathcal{R}^{+}(\beta M)\right) .
$$

(For $q=1$ one might get a semidirect product instead of a direct sum, as there is no obvious reason why the fundamental group should be abelian.) In particular, we prove the following result (see Corollary 6.7 for more details):

Theorem 1.6. Let $M_{\Sigma}$ be a spin $(L, G)$-fibered compact pseudomanifold with $L$ a simply connected homogeneous space of a compact semisimple Lie group, and $n-\ell-1 \geq 5$, where $\operatorname{dim} M=n, \operatorname{dim} L=\ell$. Assume that $M_{\Sigma}$ admits an adapted psc metric. Then the composition

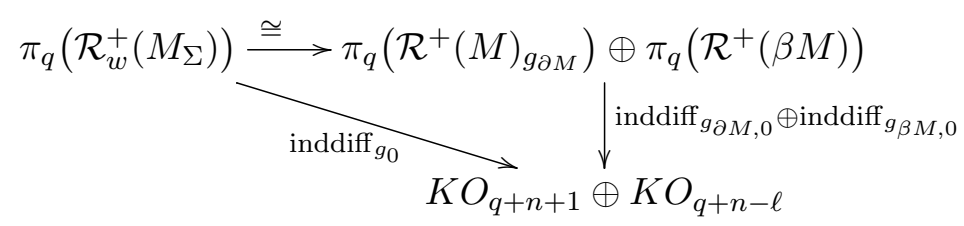

is surjective rationally and onto the 2-torsion.

We address the more general case when $M_{\Sigma}$ has non-trivial fundamental group in Corollaries 6.8 and 6.9 .

\section{$2 \quad K O$-obstructions on $L$-fibered pseudomanifolds}

\section{$2.1 K O_{n}$-classes on $L$-fibered pseudomanifolds}

Let $\left(M_{\Sigma}, g\right)$ be a Thom-Mather space of depth one, endowed with an adapted wedge metric $g$. We denote as usual by $\beta M$ the singular locus of $M_{\Sigma}$ and by $L$ the link. The resolved manifold, a manifold with fibered boundary, will be denoted by $M$.

Remark 2.1. We emphasize that for now the fibration $\phi: \partial M \rightarrow \beta M$ is assumed to be just a smooth fiber bundle with a fiber $L$, without any restriction on the structure group of that fibration. Then we say that $\left(M_{\Sigma}, g\right)$ is an $L$-fibered pseudomanifold, as opposed to an $(L, G)$ fibered pseudomanifold, which is the special case when the structure group $G$ of the fibration $\phi: \partial M \rightarrow \beta M$ acts on $L$ by isometries of a certain metric $g_{L}$. However, the relevant analytical constructions and results concerning the Dirac operators we need are well-studied and understood even for the general case of $L$-fibered pseudomanifolds. 
Recall that $M_{\Sigma}=M \cup_{\partial M}(-N(\beta M))$, where $N(\beta M)$ is the tubular neighborhood of the singular locus, which is also the total space of a fiber bundle

$$
c(L) \rightarrow N(\beta M) \longrightarrow \beta M
$$

with fiber equal to the cone over the link $L$. This also determines a smooth fiber bundle $\phi: \partial M \rightarrow$ $\beta M$ which is a restriction of the bundle (2.1) to the link $L$. We denote by $T(\partial M / \beta M) \rightarrow \beta M$ the corresponding vertical tangent bundle. As in [14, Section 2.3] (to which we refer for further details) we fix a connection on the fiber bundle $L \rightarrow \partial M \stackrel{\phi}{\rightarrow} \beta M$. The metric $g$ on $M_{\Sigma}$ restricted to the regular part of $N(\beta M)$ is assumed to have the following structure:

$$
g=\mathrm{d} r^{2}+r^{2} g_{\partial M / \beta M}+\phi^{*} g_{\beta M}+O(r)
$$

with $r$ denoting the radial variable along the cone and where the isomorphism between the horizontal bundle $H$ of the chosen connection and $\phi^{*} T(\beta M)$ has been used. (In this general setting we employ the notation $g_{X / B}$ for a metric on the vertical tangent bundle of a smooth fiber bundle $X \rightarrow B$.)

The resolved manifold $M$ inherits two metrics: the restriction of $g$ to $M$, a Riemannian metric of product type near the boundary, denoted $g_{M}$, and the extension of the metric $g$ on $\stackrel{\circ}{M} \equiv M_{\Sigma}^{\mathrm{reg}}$ to the wedge metric on the wedge tangent bundle ${ }^{w} T M \rightarrow M$. (This was defined in [4, Section 4] and in [2] under the alternate name incomplete edge tangent bundle; see again Part I [14, Section 2.3] for a quick introduction to the wedge tangent bundle). We assume that $M$, or equivalently $M_{\Sigma}^{\mathrm{reg}}$, is given a spin structure. This fixes a spin structure on $\left(\partial M, g_{\partial M}\right)$ also. Then we assume that $\beta M$ is also spin and fix a spin structure for $\left(\beta M, g_{\beta M}\right)$. This also fixes a spin structure for the vertical tangent bundle $T(\partial M / \beta M) \rightarrow \beta M$ endowed with the vertical metric $g_{\partial M / \beta M}$.

Let us recall some basic facts in spin geometry. We refer to [31, Chapter II, Section 7] for further details. Let $C \ell_{n}$ denote the Clifford algebra, and $\ell: \operatorname{Spin}_{n} \rightarrow \operatorname{Hom}\left(C \ell_{n}, C \ell_{n}\right)$ the representation given by left multiplication. Then we denote by $\Phi_{g}(M)$ the bundle given by $P_{\text {spin }} \times{ }_{\ell} C \ell_{n}$ (here $P_{\text {spin }}$ is the principal $\operatorname{Spin}(n)$-bundle defined by the spin structure). There is a fiberwise action of $C \ell_{n}$ on $\Phi_{g}(M)$ on the right which makes $\Phi_{g}(M)$ a bundle of rank one $C \ell_{n}$-modules. The bundle $\mathscr{\phi}_{g}(M)$ inherits a Levi-Civita connection $\nabla$. Let $\mathscr{P}_{g}$ be the associated $C \ell_{n}$-linear Atiyah-Singer operator; thus, by definition, $\mathfrak{P}_{g}=\mathrm{cl} \circ \nabla$. The operator $\mathfrak{P}_{g}$ has the usual local expression

$$
\mathfrak{P}_{g}=\sum_{j} \operatorname{cl}\left(e^{j}\right) \nabla_{e_{j}}
$$

with $\left\{e_{j}\right\}$ a local orthonormal frame of vector fields and $\left\{e^{j}\right\}$ the dual basis defined by the metric. $\mathscr{P}_{g}$ is a $\mathbb{Z} / 2$-graded odd formally self-adjoint operator of Dirac type commuting with the right action of $C \ell_{n}$. For this operator the Schrödinger-Lichnerowicz formula holds:

$$
\mathfrak{P}_{g}^{2}=\nabla^{*} \nabla+\frac{1}{4} \kappa_{g}
$$

with $\kappa_{g}$ denoting the scalar curvature of $g$. See again [31, Chapter II, Section 7] and also [42] for more details on this crucial point.

Notation. Unless absolutely necessary we shall omit the reference to the wedge metric $g$ in the bundle and the operator, thus denoting the $C \ell_{n}$-linear Atiyah-Singer operator simply by

$\mathfrak{P}: C^{\infty}(M, \mathscr{\phi}(M)) \rightarrow C^{\infty}(M, \mathscr{\phi}(M))$

Moreover, we shall often use the shorter notation $\mathscr{\phi}$ instead of $\mathscr{\phi}(M)$. 
As in Part I, we can regard $\mathscr{P}$ as a wedge differential operator of first order on $L^{2}(M, \mathscr{\phi})$, initially with domain equal to $C_{c}^{\infty}\left(M_{\Sigma}^{\mathrm{reg}}, \Phi\right) \subset L^{2}(M, \mathscr{\phi})$ (more on this below). We are looking for self-adjoint $C \ell_{n}$-linear extensions of this differential operator in $L^{2}(M, \mathscr{\phi})$.

We are assuming that $\partial M$ is a fiber bundle of spin manifolds, $L \rightarrow \partial M \stackrel{\varphi}{\rightarrow} \beta M$, and we fix a connection on this bundle so as to have a well-defined notion of horizontal and vertical subspaces. Consequently

$$
\mathfrak{S}(\partial M) \simeq \mathfrak{S}(\partial M / \beta M) \hat{\otimes} \varphi^{*} \mathfrak{S}(\beta M),
$$

where $\hat{\otimes}$ denotes the graded tensor product. Extending work of Bismut and Cheeger on fibrations of spin manifolds, see [9, Section 4], Albin and Gell-Redman made a careful study of the Levi-Civita connection near the singular stratum of a depth-one spin pseudomanifold - see Sections 2.2 and 3.1 in [2]; this study implies the following structure of $\mathfrak{P}$ near the singular stratum:

$$
\mathfrak{D}=\operatorname{cl}(\mathrm{d} r) \partial_{r}+\operatorname{cl}(\mathrm{d} r) \frac{\ell}{2 r}+\frac{1}{r} \mathfrak{P}_{\partial M / \beta M} \hat{\otimes} \operatorname{Id}+\operatorname{Id} \hat{\otimes} \widetilde{\mathfrak{P}}_{\beta M}+\mathfrak{B}
$$

with $\ell=\operatorname{dim} L, \mathfrak{P}_{\partial M / \beta M}$ the vertical family of Atiyah-Singer operators on the fibration

$$
L \rightarrow \partial M \stackrel{\varphi}{\rightarrow} \beta M,
$$

$\widetilde{\mathfrak{P}}_{\beta M}$ an explicit horizontal operator, and $\mathfrak{B}$ a bundle endomorphism which is $O(r)$. Formula $(2.2)$ can be rewritten as

$$
\mathfrak{P}=r^{-1}\left(\operatorname{cl}(\mathrm{d} r) r \partial_{r}+\operatorname{cl}(\mathrm{d} r) \frac{\ell}{2}+\mathfrak{P}_{\partial M / \beta M} \hat{\otimes} \operatorname{Id}+r \operatorname{Id} \hat{\otimes} \widetilde{\mathfrak{P}}_{\beta M}+r \mathfrak{B}\right)
$$

and exhibits $\mathscr{D}$ as a wedge differential operator of order $1: \mathfrak{D}=r^{-1} \mathfrak{P}^{e}$, with $\mathfrak{P}^{e}$ an edge differential operator. See Part I and of course [2] for an introduction to edge and wedge operators. With a small abuse of notation, widely used in family index theory, we denote by $\mathfrak{P}_{L}$ the generic operator of the vertical family $\mathfrak{P}_{\partial M / \beta M}$ and by $\operatorname{spec}_{L^{2}}\left(\mathfrak{D}_{L}\right)$ its spectrum.

The following result has been discussed in Part I:

Theorem 2.2. Assume that

$$
\operatorname{spec}_{L^{2}}\left(\mathfrak{P}_{L}\right) \cap(-1 / 2,1 / 2)=\varnothing \quad \text { for each fiber } L .
$$

Then the following holds:

(i) The operator $\mathfrak{P}$ with domain $C_{c}^{\infty}\left(M_{\Sigma}^{\mathrm{reg}}, \mathscr{\phi}\right) \subset L^{2}(M, \Phi)$ is essentially self-adjoint.

(ii) Its unique self-adjoint extension, still denoted by $\mathfrak{P}$, defines a $C \ell_{n}$-linear Fredholm operator and thus a class $\alpha_{w}\left(M_{\Sigma}, g\right)$ in $K O_{n}$, with $n=\operatorname{dim} M_{\Sigma}$.

As explained in Part I, $(i)$ and $(i i)$ are direct consequences of the analysis developed in [2] (which builds in turn on the slightly more complicated case of the signature operator on Witt spaces, see [4]). The main step is the construction of a $C l_{n}$-linear parametrix for an operator $\mathfrak{P}$ satisfying (2.3); this is based crucially on the construction of a parametrix for the edge operator $\mathfrak{P}^{e}$ associated to $\mathfrak{P}$, using Mazzeo's edge pseudodifferential calculus [35].

The following result also follows directly from [2] and from the Schrödinger-Lichnerowicz formula, which is valid for $\mathfrak{P}$ :

Theorem 2.3. If the tubular neighborhood of the singular stratum $\left(N(\beta M),\left.g\right|_{N(\beta M)}\right)$ has nonnegative scalar curvature, then (2.3) holds. See [2, Theorem 1.3].

If $\left(M_{\Sigma}^{\mathrm{reg}}, g\right)$ has psc everywhere then (2.3) holds (this is clear from above, given that $(N(\beta M)$, $\left.\left.g\right|_{N(\beta M)}\right)$ has psc) and the unique self-adjoint extension $\mathfrak{D}$ is $L^{2}$-invertible; in particular

$$
\alpha_{w}\left(M_{\Sigma}, g\right)=0 \quad \text { in } K O_{n} .
$$


Definition 2.4. We shall say that the stratified spin pseudomanifold $\left(M_{\Sigma}, g\right)$ is geometric-Witt if the metric $g$ is such that $\operatorname{spec}_{L^{2}}\left(\not_{L}\right) \cap(-1 / 2,1 / 2)=\varnothing$ for each fiber $L$.

This notion is taken from [2], where it is applied to any generalized wedge Dirac operator. If $\left(M_{\Sigma}, g\right)$ does not satisfy (2.3) but is such that the vertical metric $g_{\partial M / \beta M}$ is of psc along the fibers, then we can still define a wedge-alpha class $\alpha_{w}\left(M_{\Sigma}, g\right) \in K O_{n}$. Indeed, by the Schrödinger-Lichnerowicz formula applied to the vertical family we know that there exists $\epsilon>0$ such that

$$
\operatorname{spec}_{L^{2}}\left(\mathfrak{P}_{L}\right) \cap(-\epsilon, \epsilon)=\varnothing \quad \text { for each fiber } L .
$$

From (2.4) we can achieve condition (2.3) above by rescaling the vertical metric $g_{\partial M / \beta M}$. (This requires some adjustments in order to glue the rescaled metric around the singular locus $\beta M$ to the original metric on the complement of $N(\beta M)$, along the lines of [16, Appendix 6].) We obtain in this way a wedge-alpha class for $\mathfrak{D}$ by considering the wedge-alpha class of the operator associated to the rescaled metric.

There is a different (and in fact preferable) realization of this class that makes use of a natural self-adjoint domain defined for any wedge Dirac operator with invertible vertical family along the fibers of the boundary fibration. This is the so called vertical APS domain, see [1, Definition 2.3]. By applying the definition to our case we thus obtain a closed self-adjoint extension of $\mathfrak{P}$, denoted $\mathcal{D}_{\text {VAPS }}(\mathfrak{D})$. Following [1] one proves that on this domain $\mathfrak{D}$ admits a parametrix, that is, an inverse modulo compacts, which can be used in order to see that $\left(\mathfrak{P}, \mathcal{D}_{\text {VAPS }}(\mathfrak{P})\right)$ is a $C \ell_{n^{-}}$ linear Fredholm operator on its domain endowed with the graph norm. We obtain in this way a class $\alpha_{w}\left(M_{\Sigma}, g\right) \in K O_{n}$; one can prove that the class defined through the vertical APS domain and the class defined by the operator associated to the rescaled metric are in fact equal. See [1, Remark 4.10] for a sketch of the argument.

Definition 2.5. We shall say that the stratified spin pseudomanifold $\left(M_{\Sigma}, g\right)$ is psc-Witt if the metric $g$ is of psc along the links, i.e., if the vertical metric $g_{\partial M / \beta V}$ induces on each fiber $L$ a metric of psc.

Given a psc-Witt stratified spin pseudomanifold $\left(M_{\Sigma}, g\right)$ we define its wedge alpha class in $K O_{n}$ by considering the $C \ell_{n}$-linear Fredholm operator $\left(\mathfrak{P}, \mathcal{D}_{\mathrm{VAPS}}(\mathfrak{P})\right)$.

Remark 2.6. In this article, which concentrates on $(L, G)$-pseudomanifolds, with $L=G / H$ a simply connected homogeneous space and $G$ a compact semisimple Lie group acting on $L$ by isometries, we do not need to consider the vertical APS domain $\mathcal{D}_{\text {VAPS }}(\not{D})$ or, equivalently, the rescaled metric. Indeed, the proof of [2, Theorem 1.3] shows that (2.3) is automatic when scal $_{g_{L}}=\operatorname{scal}_{S^{\ell}}=\ell(\ell-1)$, which is the normalization we have adopted, see (1.1). Put it differently, for the purposes of this article we can and we shall exclusively treat the geometricWitt case.

Theorem 2.7. Let $\left(M_{\Sigma}, g\right)$ be a geometric-Witt spin pseudomanifold. Then there is a welldefined fundamental class $\left[\mathfrak{D}_{g}\right] \in K O_{n}\left(M_{\Sigma}\right)$.

Proof. Following [19, Section 11], [40, Section 7.1] consider $\mathcal{A}$, the algebra of smooth functions on $M$ that are constant along the fibers of $\partial M \stackrel{\varphi}{\rightarrow} \beta M$; there is a dense inclusion $\mathcal{A} \hookrightarrow C\left(X_{\Sigma}\right)$. Once a parametrix for $\mathfrak{D}$ is constructed, one obtains the existence of the fundamental class in $K O_{n}\left(M_{\Sigma}\right)$ by proceeding exactly as for the signature operator on Witt spaces - see [4, Section 6.2], but taking $\mathcal{A}$ as a dense subalgebra of $C\left(M_{\Sigma}\right)$ instead of the subalgebra of Lipschitz functions employed in [4, Section 6.2]. The details are very similar and thus we omit them.

Remark 2.8. As usual, we have $\alpha_{w}(M, g)=\pi_{*}\left[\mathscr{P}_{g}\right]$, with $\pi$ the mapping of the compact pseudomanifold $M_{\Sigma}$ to a point. 
Remark 2.9. We remark that the wedge $\alpha$-class and the fundamental class $\left[\mathfrak{D}_{g}\right]$ are unchanged if $g(t), t \in[0,1]$, is a 1-parameter family of adapted wedge metrics that are geometric-Witt for any $t \in[0,1]$. This is in fact a special case of Theorem 2.17 below.

Remark 2.10. Theorem 2.7, Remarks 2.8 and 2.9 also hold when $\left(M_{\Sigma}, g\right)$ is psc-Witt, with the condition in Remark 2.9 being that $g(t)$ is psc-Witt for each $t \in[0,1]$. As we shall not use this case, we do not discuss the details.

\section{$2.2 \quad C^{*}$-algebras and $K O$ classes associated to $\not{D}$}

In this second part of our work we would like to bring in the fundamental group of the pseudomanifold $M_{\Sigma}$. In fact, we will begin, more generally, with Galois $\Gamma$-covering spaces since this approach is more general and simplifies the techniques.

Let $\Gamma$ be a discrete group. A $\Gamma$-cover of $M_{\Sigma}$ will be denoted by $M_{\Sigma}^{\Gamma} \stackrel{\pi}{\rightarrow} M_{\Sigma}$. We shall keep the notation $\widetilde{M}_{\Sigma} \rightarrow M_{\Sigma}$ for the universal cover of $M_{\Sigma}$. We notice that the $\Gamma$-cover $M_{\Sigma}^{\Gamma}$ has a natural structure of depth-1 pseudomanifold, with strata equal to the inverse image of the strata of $M_{\Sigma}$ through the projection map $\pi$. See [40] and [39] for more on this background material. Hence, the singular stratum $\beta M^{\Gamma}$ of $M_{\Sigma}^{\Gamma 1}$ is a $\Gamma$-cover of $\beta M$,

$$
\Gamma \rightarrow \beta M^{\Gamma} \rightarrow \beta M
$$

with a link diffeomorphic again to $L$; similarly, the regular part $\left(M_{\Sigma}^{\Gamma}\right)^{\mathrm{reg}}$ of $M_{\Sigma}^{\Gamma}$ is a $\Gamma$-cover of the regular part $M_{\Sigma}^{\text {reg }}$ of $M_{\Sigma}$. Even if our Galois cover is the universal cover of $M_{\Sigma}$, then $\beta M^{\Gamma}$ need not be, in general, the universal covering $\widetilde{\beta M}$ of $\beta M$. A similar remark applies to the two regular parts.

Finally, if $M^{\Gamma}$ is the resolution of $M_{\Sigma}^{\Gamma}$ then it is easy to see that $M^{\Gamma}$ is in a natural way a Galois covering of $M$. The interior of $M^{\Gamma}$ is clearly a Galois $\Gamma$-cover of the interior of $M$. If

$$
L \rightarrow \partial M \stackrel{p}{\rightarrow} \beta M
$$

is the boundary fibration of $M$ and $L \rightarrow \partial M^{\Gamma} \stackrel{p_{\Gamma}}{\longrightarrow} \beta M^{\Gamma}$ is the boundary fibration of $M^{\Gamma}$ and if $\pi$ denotes the quotient map induced by the action of $\Gamma$, then there is a commutative diagram

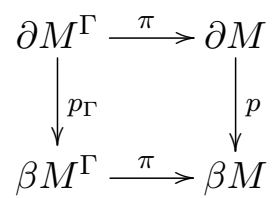

with $\partial M^{\Gamma} \stackrel{\pi}{\rightarrow} \partial M$ inducing a diffeomorphism between the fiber of $p_{\Gamma}$ over $x \in \beta M^{\Gamma}$ and the fiber of $p$ over $\pi(x)$. In the sequel we shall always endow $M_{\Sigma}^{\Gamma}$, or rather its regular part, with the lift of an adapted wedge metric on $M$; this metric extends as a $\Gamma$-equivariant wedge metric on all of the resolution $M^{\Gamma}$; we denote this $\Gamma$-invariant metric by $g_{\Gamma}$. Let us assume once again that $M$ and $\beta M$ are spin; then also $M^{\Gamma}$ and $\beta M^{\Gamma}$ will be spin. Consequently, $M_{\Sigma}^{\mathrm{reg}}$ and $\left(M_{\Sigma}^{\Gamma}\right)^{\mathrm{reg}}$ are spin.

Let $\mathfrak{P}_{\Gamma}$ be the associated $\Gamma$-equivariant $C l_{n}$-linear Atiyah-Singer operator. Notice that since the link of $M_{\Sigma}^{\Gamma}$ is again $L$ the analysis to be developed for understanding the properties of $\mathfrak{P}_{\Gamma}$ is obtained by combining the usual analysis of $\Gamma$-equivariant operators on $\Gamma$-covers of smooth compact manifolds and the wedge analysis on compact stratified spaces. This principle is explained in detail in [39].

\footnotetext{
${ }^{1}$ As we have denoted the singular locus of $M_{\Sigma}$ by $\beta M$, we denote the singular locus of $M_{\Sigma}^{\Gamma}$ by $\beta M^{\Gamma}$.
} 
For the next theorem, however, we shall rather use the Mishchenko-Fomenko operator associated to $\mathfrak{P}$. Define the Mishchenko bundle $\mathcal{V}:=M_{\Sigma}^{\Gamma} \times_{\Gamma} C_{r, \mathbb{R}}^{*}(\Gamma)$, a bundle of finitely generated projective $C_{r, \mathbb{R}}^{*}(\Gamma)$-modules of rank 1 over the whole $M_{\Sigma}$. By definition $\mathscr{P}_{\mathrm{MF}}$ is equal to $\mathscr{D}$ twisted by $\mathcal{V}$ restricted to the regular part of $M_{\Sigma}$. We shall also refer to this operator as the Atiyah-Singer-Mishchenko operator and if necessary we shall denote it more precisely by $\mathfrak{P}_{\mathrm{MF}, g}$. We shall also consider the associated edge-operator, $\mathfrak{P}_{\mathrm{MF}}^{e}$, obtained by twisting $\mathfrak{P}^{e}$ by the Mishchenko bundle $\mathcal{V}$.

Theorem 2.11. Let $M$ and $\beta M$ be spin and let $g$ be a wedge adapted metric. Consider a Galois $\Gamma$-cover $M_{\Sigma}^{\Gamma}$ of $M_{\Sigma}$ and endow the regular part with the associated $\Gamma$-equivariant metric $g_{\Gamma}$ as above. Let us assume that $\left(M_{\Sigma}, g\right)$ is geometric-Witt. Consider the Atiyah-Singer-Mishchenko operator $\mathfrak{P}_{\mathrm{MF}, g}$. Then there is a unique self-adjoint extension of $\mathfrak{D}_{\mathrm{MF}, g}$, with domain denoted $\mathcal{D}\left(\mathfrak{P}_{\mathrm{MF}, g}\right)$ such that the following hold:

(1) the pair $\left(\mathfrak{P}_{\mathrm{MF}, g}, \mathcal{D}\left(\mathfrak{P}_{\mathrm{MF}, g}\right)\right)$ defines an unbounded Kasparov $\left(\mathbb{R}, C_{r, \mathbb{R}}^{*} \Gamma \otimes C \ell_{n}\right)$-bimodule and thus an index class $\operatorname{Ind}_{w}\left(\mathfrak{P}_{\mathrm{MF}, g}, M_{\Sigma}^{\Gamma}\right) \in K K O_{n}\left(\mathbb{R}, C_{r, \mathbb{R}}^{*} \Gamma\right)$; as usual we identify the group $K K O_{n}\left(\mathbb{R}, C_{r, \mathbb{R}}^{*} \Gamma\right)$ with the isomorphic group $K O_{n}\left(C_{r, \mathbb{R}}^{*} \Gamma\right)$,

(2) if $g$ has psc everywhere then $\operatorname{Ind}_{w}\left(\mathfrak{P}_{\mathrm{MF}, g}, M_{\Sigma}^{\Gamma}\right)=0$ in $K O_{n}\left(C_{r, \mathbb{R}}^{*} \Gamma\right)$,

(3) if ass: $K O_{n}(B \Gamma) \rightarrow K O_{n}\left(C_{r, \mathbb{R}}^{*} \Gamma\right)$ is the assembly map and if $f: M_{\Sigma} \rightarrow B \Gamma$ is the classifying map of the $\Gamma$-cover, then

$$
\operatorname{ass}\left(f_{*}\left[\mathfrak{P}_{g}\right]\right)=\operatorname{Ind}_{w}\left(\mathfrak{P}_{\mathrm{MF}, g}, M_{\Sigma}^{\Gamma}\right) \quad \text { in } \quad K O_{n}\left(C_{r, \mathbb{R}}^{*} \Gamma\right) .
$$

Sketch of the proof. The proof is an easy adaptation of the corresponding result for the signature operator on Witt spaces, see [4, Proposition 6.4, Theorem 6.6], and for this reason we shall be brief. The operator $\mathfrak{D}_{\mathrm{MF}}$ acts on the sections of $\Phi \otimes \mathcal{V}$. Since the Mishchenko bundle on the tubular neighborhood of the singular locus $\beta M$ is the pull-back of a bundle on $\beta M$, we see that the analysis to be developed in order to understand $\mathfrak{D}_{\mathrm{MF}}$ is no more difficult than the one already developed for $\mathfrak{D}$. More precisely, following the proof of [4, Proposition 6.4], we see that $N_{q}\left(\mathfrak{P}_{\mathrm{MF}}^{e}\right)$, the normal operator of $\mathfrak{P}_{\mathrm{MF}}^{e}$ at $q \in \beta M$ is equal, up to conjugation by a bundle isomorphism, to $N_{q}(\mathfrak{P}) \otimes \operatorname{Id}_{C_{r, \mathbb{R}}^{*} \Gamma}$ and so its invertibility properties, that are crucial in the analysis developed in [2], are a consequence of those already established for $N_{q}(\mathfrak{P})$. The proof now proceeds parallel to the one given in [4, Section 6.3] for the signature operator on Witt spaces. Notice that in (2) we use again the fact that a metric which is psc everywhere is psc in $\left(N(\beta M),\left.g\right|_{N(\beta M)}\right)$ and thus geometric-Witt.

Notation. We shall briefly denote the index class $\operatorname{Ind}_{w}\left(\mathfrak{P}_{\mathrm{MF}, g}, M_{\Sigma}^{\Gamma}\right)$ as

$$
\alpha_{w}^{\Gamma}\left(M_{\Sigma}, g\right) \in K O_{*}\left(C_{r, \mathbb{R}}^{*} \Gamma\right) .
$$

In case we want to be very precise about the Galois cover $M_{\Sigma}^{\Gamma}$ involved in the definition of this class we shall also write

$$
\alpha_{w}^{\Gamma}\left(M_{\Sigma}, g, f\right) \in K O_{*}\left(C_{r, \mathbb{R}}^{*} \Gamma\right), \quad \text { where } \quad f: M_{\Sigma} \rightarrow B \Gamma \quad \text { and } \quad M_{\Sigma}^{\Gamma}=f^{*} E \Gamma .
$$

Remark 2.12. Similarly to Remark 2.9 , the class $\alpha_{w}^{\Gamma}\left(M_{\Sigma}, g\right) \in K O_{*}\left(C_{r, \mathbb{R}}^{*} \Gamma\right)$ is unchanged if $g(t), t \in[0,1]$, is a 1-parameter family of adapted wedge metrics that are geometric-Witt for any $t \in[0,1]$. This is in fact a special case of Theorem 2.17 below.

Remark 2.13. The above results can also be established for psc-Witt pseudomanifold, either by using the vertical APS domain or by rescaling in the fiber direction on the boundary. We shall not need this more general case. 
Remark 2.14. The existence of a fundamental class $[\mathfrak{P}] \in K_{*}\left(M_{\Sigma}\right)$ and of a class $\alpha^{\Gamma}\left(M_{\Sigma}, g\right)$ can be established more generally for spin pseudomanifolds of arbitrary depth, assuming of course suitable Witt conditions along the links. This is based heavily on the general edge pseudodifferential calculus developed by Albin and Gell-Redman in [1]. Details will appear in [3].

\subsection{Cylindrical $\mathrm{KO}$-theory classes and a gluing formula}

We decompose

$$
M_{\Sigma}=M \cup_{\partial M}(-N(\beta M)) \quad \text { and } \quad M_{\Sigma}^{\mathrm{reg}}=M \cup_{\partial M}\left(-N(\beta M)^{\mathrm{reg}}\right) .
$$

Let $g$ be an adapted wedge metric on $M_{\Sigma}$. Denote by $g_{M}$ the Riemannian metric equal to the restriction of $g$ to $M$ and by $g_{N(\beta M)}$ the metric equal to the restriction of $g$ to $N(\beta M)$, the collar neighborhood of the singular stratum. By assumption,

$$
g_{N(\beta M)}=\mathrm{d} r^{2}+r^{2} g_{\partial M / \beta M}+p^{*} g_{\beta M}+O(r) .
$$

Recall that an adapted wedge metric $g$ is such that $g_{M}$ and $g_{N(\beta M)}$ are of product type in a collar neighborhood of $\partial M$. We make the hypothesis that $g$ is geometric-Witt and that the whole metric $g_{\partial M}$ is of psc. Now attach an infinite cylinder to $M$ along the boundary $\partial M$ and extend the metric to be constant on the cylinder; similarly, attach an infinite cylinder to $N(\beta M)$ and extend the metric. Because of the hypothesis that $g_{\partial M}$ is of psc we have well defined classes

$$
\operatorname{Ind}_{\mathrm{cyl}}\left(\mathfrak{P}_{\mathrm{MF}, g_{M}}, M^{\Gamma}\right), \quad \operatorname{Ind}_{\mathrm{cyl}, w}\left(\mathfrak{P}_{\mathrm{MF}, g_{N(\beta M)}}, N(\beta M)^{\Gamma}\right) \quad \text { in } \quad K O_{*}\left(C_{r, \mathbb{R}}^{*} \Gamma\right) .
$$

For the existence of the cylindrical class on $M^{\Gamma}$ we refer the reader to $[18,33,47]$ and references therein. The existence of the index class $\operatorname{Ind}_{\mathrm{cyl}, w}\left(\mathscr{P}_{\mathrm{MF}}, N(\beta M)^{\Gamma}\right)$ follows from the above theorem and these references.

Notation. We shall briefly denote the above index classes as

$$
\alpha_{\text {cyl }}^{\Gamma}\left(M, g_{M}\right) \quad \text { and } \quad \alpha_{\text {cyl }, w}^{\Gamma}\left(N(\beta M), g_{N(\beta M)}\right) \in K O_{*}\left(C_{r, \mathbb{R}}^{*} \Gamma\right) .
$$

Proposition 2.15. Under the same assumptions as above, namely that $g$ is geometric-Witt and that $\left.g\right|_{\partial M}$ is of psc, the following gluing formula holds:

$$
\alpha_{w}^{\Gamma}\left(M_{\Sigma}, g\right)=\alpha_{\mathrm{cyl}}^{\Gamma}\left(M, g_{M}\right)+\alpha_{\mathrm{cyl}, w}^{\Gamma}\left(N(\beta M), g_{N(\beta M)}\right) \quad \text { in } \quad K O_{*}\left(C_{r, \mathbb{R}}^{*} \Gamma\right) .
$$

Consequently, if $\left(N(\beta M), g_{N(\beta M)}\right)$ if of psc then

$$
\alpha_{w}^{\Gamma}\left(M_{\Sigma}^{\Gamma}, g\right)=\alpha_{\text {cyl }}^{\Gamma}\left(M^{\Gamma}, g_{M}\right) \quad \text { in } \quad K O_{*}\left(C_{r, \mathbb{R}}^{*} \Gamma\right) .
$$

Proof. The existence of these classes has been discussed above. The gluing formula has been discussed for the signature operator on Witt and Cheeger spaces by Albin-Piazza [5], based on a well known technique due to Bunke - see [18]; the same arguments, with minor modifications, apply here.

\subsection{Spin bordism of geometric-Witt pseudomanifolds}

It is well-known that in the smooth context the Fredholm index of the spin-Dirac operator and the index class of the spin-Dirac operator twisted by the Mishchenko bundle define group homomorphisms

$$
\text { ind: } \Omega_{n}^{\text {spin }} \rightarrow \mathbb{Z}, \quad \operatorname{Ind}_{\Gamma}: \Omega_{n}^{\text {spin }}(B \Gamma) \rightarrow K_{*}\left(C_{r}^{*} \Gamma\right),
$$


where for the numeric index we limit ourselves to $n=4 k$. More generally, the $\alpha$ invariant of the Atiyah-Singer operator $\mathfrak{D}$ defines a group homomorphism

$$
\alpha: \Omega_{n}^{\text {spin }} \rightarrow K O_{n}
$$

while the $\alpha^{\Gamma}$-invariant of the Atiyah-Singer-Mishchenko operator $\mathfrak{P}_{\mathrm{MF}}$ defines a group homomorphism

$$
\alpha^{\Gamma}: \Omega_{n}^{\mathrm{spin}}(B \Gamma) \rightarrow K O_{*}\left(C_{r, \mathbb{R}}^{*} \Gamma\right)
$$

The results for the spin-Dirac operator, see (2.6), is well known and treated in several references. See for example the detailed treatment in [7] and its extension to the $C_{r}^{*} \Gamma$-index class in [32]. For the passage to the Atiyah-Singer operator, see [31, Chapter IV, Section 4, equation (4.3)] and [42] or, for a purely analytic argument, the appendix to this paper, Section A.

Definition 2.16. Let $\left(M_{\Sigma}, g\right)$ and $\left(M_{\Sigma}^{\prime}, g^{\prime}\right)$ be two spin-stratified pseudomanifolds of dimension $n$ and let us assume that they are both geometric-Witt. Let $\left(W_{\Sigma}, \bar{g}\right)$ be a spin-stratified pseudomanifold of dimension $(n+1)$ with collared boundary and adapted wedge metric, also of product-type near the boundary, such that $\partial W_{\Sigma}=M_{\Sigma} \cup\left(-M_{\Sigma}^{\prime}\right)$ and $\left.\bar{g}\right|_{\partial W_{\Sigma}}=g \cup g^{\prime}$. We shall say that $\left(W_{\Sigma}, \bar{g}\right)$ provides a geometric-Witt bordism between $\left(M_{\Sigma}, g\right)$ and $\left(M_{\Sigma}^{\prime}, g^{\prime}\right)$ if the adapted wedge metric $\bar{g}$ is globally psc-Witt.

If $\Gamma$ is a discrete group as above and $f: M_{\Sigma} \rightarrow B \Gamma$ and $f^{\prime}: M_{\Sigma}^{\prime} \rightarrow B \Gamma$ are classifying maps, then we shall say that $\left(M_{\Sigma}, g, f\right)$ is geometric-Witt bordant to $\left(M_{\Sigma}^{\prime}, g^{\prime}, f^{\prime}\right)$ if there exists $\left(W_{\Sigma}, \bar{g}\right)$ as above and a continuous map $F: W_{\Sigma} \rightarrow B \Gamma$ restricting to $f$ and $f^{\prime}$ on the boundary. We use the notation $\left(W_{\Sigma}, \bar{g}, F\right):\left(M_{\Sigma}, g, f\right) \rightsquigarrow\left(M_{\Sigma}^{\prime}, g^{\prime}, f^{\prime}\right)$ for such a geometric-Witt bordism. Notice that $f, f^{\prime}$ and $F$ define in a natural way Galois coverings $M_{\Sigma}^{\Gamma},\left(M_{\Sigma}^{\prime}\right)^{\Gamma}, W_{\Sigma}^{\Gamma}$ and thus Mishchenko bundles on $M_{\Sigma}, M_{\Sigma}^{\prime}$ and $W_{\Sigma}$. We denote briefly by $\mathfrak{P}_{\mathrm{MF}}$ and $\mathfrak{P}_{\mathrm{MF}}^{\prime}$ the corresponding AtiyahSinger-Mishchenko operators on $M_{\Sigma}$ and $M_{\Sigma}^{\prime}$.

Theorem 2.17. If there exists a geometric-Witt bordism $\left(W_{\Sigma}, \bar{g}\right):\left(M_{\Sigma}, g\right) \rightsquigarrow\left(M_{\Sigma}^{\prime}, g^{\prime}\right)$, then

$$
\alpha_{w}\left(M_{\Sigma}, g\right)=\alpha_{w}\left(M_{\Sigma}^{\prime}, g^{\prime}\right) \quad \text { in } \quad K O_{n} .
$$

If there exists a geometric-Witt bordism $\left(W_{\Sigma}, \bar{g}, F\right):\left(M_{\Sigma}, g, f\right) \rightsquigarrow\left(M_{\Sigma}^{\prime}, g^{\prime}, f^{\prime}\right)$, then

$$
\alpha_{w}^{\Gamma}\left(M_{\Sigma}, g, f\right)=\alpha_{w}^{\Gamma}\left(M_{\Sigma}^{\prime}, g^{\prime}, f^{\prime}\right) \quad \text { in } \quad K O_{n}\left(C_{r, \mathbb{R}}^{*} \Gamma\right)
$$

where we recall that the above classes are defined in terms of $M_{\Sigma}^{\Gamma}=f^{*} E \Gamma$ and $\left(M_{\Sigma}^{\prime}\right)^{\Gamma}=\left(f^{\prime}\right)^{*} E \Gamma$.

Sketch of proof. Once an analytic proof of the spin-bordism invariance is given in the smooth closed case, the argument for geometric-Witt pseudomanifolds is the same. For this reason, we just give a sketch of the argument. Indeed, by general principles, the proof is reduced to a collar neighborhood of the boundary where the two directions, the one of the boundary and the one normal to the boundary, are completely decoupled. Now, for smooth spin manifolds we have provided a purely analytic proof of the spin-bordism invariance of the $\alpha$-class and of the $\alpha^{\Gamma}$-class in the appendix; ${ }^{2}$ it is very easy to extend this proof to the geometric-Witt case, exactly as it is done for the signature operator on Witt pseudomanifolds in order to establish the Witt-bordism invariance of the signature index and of the $C_{r}^{*} \Gamma$ signature-index class. See the proof of [4, Theorem 7.1].

Remark 2.18. One can extend Definition 2.16 to the psc-Witt case and the analogue of Theorem 2.17 holds.

\footnotetext{
${ }^{2}$ This is well known to the experts, but we could not pin down a quotable reference.
} 


\section{$3 \quad K O$-obstructions on $(L, G)$-fibered pseudomanifolds}

In this section we shall finally meet the obstructions to the existence of a wedge metric of psc on an $(L, G)$-fibered pseudomanifold. We treat first the general case of $L$-fibered pseudomanifolds and then specialize to the case of $(L, G)$-fibered pseudomanifolds; the latter are the pseudomanifolds for which we shall prove an existence theorem.

\subsection{KO-obstructions for general $L$-fibered pseudomanifolds}

Let $\Gamma$ be a finitely generated discrete group, and assume that $M_{\Sigma}^{\Gamma}$ is a $\Gamma$-cover of an $L$-fibered pseudomanifold $M_{\Sigma}=M \cup_{\partial M}(-N(\beta M))$. If $M_{\Sigma}$ admits an adapted wedge metric of psc then we know that $M_{\Sigma}$ is geometric-Witt and $\alpha_{w}^{\Gamma}\left(M_{\Sigma}, g\right)=0$ in $K O_{*}\left(C_{r, \mathbb{R}}^{*}(\Gamma)\right)$. See Theorems 2.3 and $2.11(2)$. Since $\left(N(\beta M), g_{N(\beta M)}\right)$ is of psc we also have that $\alpha_{w}^{\Gamma}\left(M_{\Sigma}, g\right)=\alpha_{\text {cyl }}^{\Gamma}\left(M, g_{M}\right)$ in $K O_{*}\left(C_{r, \mathbb{R}}^{*} \Gamma\right)$. See Proposition 2.15, and in particular (2.5).

Summarizing, if $g$ is an adapted wedge metric of psc on an $L$-fibered pseudomanifold of dimension $n$, then the following necessary condition is fulfilled:

$$
\alpha_{w}^{\Gamma}\left(M_{\Sigma}, g\right)=\alpha_{\text {cyl }}^{\Gamma}\left(M, g_{M}\right)=0 \quad \text { in } \quad K O_{n}\left(C_{r, \mathbb{R}}^{*}(\Gamma)\right) .
$$

Consider now the singular locus of $M_{\Sigma}^{\Gamma}$, denoted $\beta M^{\Gamma}$. We already observed that the covering map $M_{\Sigma}^{\Gamma} \rightarrow M_{\Sigma}$ induces a $\Gamma$-cover $\beta M^{\Gamma} \rightarrow \beta M$. Consider the Atiyah-Singer operator on $\left(\beta M, g_{\beta M}\right)$ and let us denote it by $\mathfrak{P}_{g_{\beta M}}^{\beta}$. We also have an Atiyah-Singer-Mishchenko operator $\mathfrak{P}_{\mathrm{MF}, g_{\beta M}}^{\beta}$, obtained by twisting $\mathfrak{P}_{g_{\beta M}}^{\beta}$ with the Mishchenko bundle associated to the $\Gamma$-cover $\Gamma \rightarrow \beta M^{\Gamma} \rightarrow \beta M$. We can consider $\operatorname{Ind}\left(\mathfrak{P}_{\mathrm{MF}, g_{\beta M}}^{\beta}, \beta M^{\Gamma}\right)$ in $K O_{n-\ell-1}\left(C_{r, \mathbb{R}}^{*}(\Gamma)\right)$ that we denote, as usual, by $\alpha^{\Gamma}\left(\beta M, g_{\beta M}\right)$. Since $\beta M$ is a closed smooth spin manifold we can in fact adopt the notation $\alpha^{\Gamma}(\beta M) \in K O_{n-\ell-1}\left(C_{r, \mathbb{R}}^{*}(\Gamma)\right)$, given that this class does not depend on the particular choice of the metric $g_{\beta M}$. See [42]. Unless further assumptions are made on the fibration $L \rightarrow \partial M \rightarrow \beta M$ we cannot infer that also $\alpha^{\Gamma}(\beta M)=0$ in $K O_{n-\ell-1}\left(C_{r, \mathbb{R}}^{*}(\Gamma)\right)$, where $\ell=\operatorname{dim} L$.

In the next section, on the other hand, we shall specialize this discussion to the case of $(L, G)$ fibered pseudomanifolds and get consequently more precise information.

\section{$3.2 K O$-classes on $(L, G)$-fibered pseudomanifolds}

We follow the notation of the previous section but we now assume that $M_{\Sigma}$ is an $(L, G)$-fibered pseudomanifold endowed with an adapted wedge metric $g$. We know that $\left(M_{\Sigma}, g\right)$ is geometricWitt - see Remark 2.6. By Theorem 2.11 there exists a well defined wedge-alpha class $\alpha_{w}^{\Gamma_{w}}\left(M_{\Sigma}, g\right)$ in $K O_{n}\left(C_{r, \mathbb{R}}^{*}(\Gamma)\right)$. Moreover, from Theorem 3.5 of Part 1 (item (1)) we know that $\left.g\right|_{\partial M}$ is of psc and so we also have a cylindrical class $\alpha_{\text {cyl }}^{\Gamma}\left(M, g_{M}\right) \in K O_{n}\left(C_{r, \mathbb{R}}^{*}(\Gamma)\right)$. As already remarked in the Introduction, the space of adapted wedge metrics on an $(L, G)$-fibered pseudomanifold is contractible. Thus $\alpha_{w}^{\Gamma}\left(M_{\Sigma}, g\right) \in K O_{n}\left(C_{r, \mathbb{R}}^{*}(\Gamma)\right)$ does not depend on the choice of the adapted wedge metric $g$. See Remark 2.12. Similarly, the cylindrical $\alpha$ class $\alpha_{\text {cyl }}^{\Gamma}\left(M, g_{M}\right)$ does not depend on the choice of $g$; indeed, if $g$ and $g^{\prime}$ are two adapted wedge metrics joined by a 1-parameter family of adapted wedge metric, then $g_{M}$ and $g_{M}^{\prime}$ are joined by a path of metrics that are uniformly of psc on the boundary $\partial M$. The result then follows from well-known properties of index classes on manifolds with cylindrical ends. See for example [26] or [34]. Thus we can adopt the notation

$$
\alpha_{w}^{\Gamma}\left(M_{\Sigma}\right) \quad \text { and } \quad \alpha_{\text {cyl }}^{\Gamma}(M)
$$

for the two classes. 
Finally, we remark that under the assumption that $\left(\beta M, g_{\beta M}\right)$ is of $p s c$, the equality

$$
\alpha_{w}^{\Gamma}\left(M_{\Sigma}, g\right)=\alpha_{\text {cyl }}^{\Gamma}\left(M, g_{M}\right)
$$

holds generally; indeed, in this case the wedge metric $g$ restricted to $N(\beta M)$ has psc - see again Theorem 3.5 of Part I (item (2)) - and thus the result follows from Proposition 2.15.

Assume now that our $(L, G)$-fibered pseudomanifolds is endowed with an adapted wedge metric of psc. We then certainly have that

$$
\alpha_{w}^{\Gamma}\left(M_{\Sigma}, g\right)=\alpha_{\text {cyl }}^{\Gamma}\left(M, g_{M}\right)=0 \quad \text { in } \quad K O_{n}\left(C_{r, \mathbb{R}}^{*}(\Gamma)\right)
$$

since this is true even in the general $L$-fibered case. However, in this particular case, because of Theorem 3.5 of Part I (item (3)), we have additionally that

$$
\alpha^{\Gamma}(\beta M)=0 \in K O_{n-\ell-1}\left(C_{r, \mathbb{R}}^{*}(\Gamma)\right) .
$$

This discussion proves the obstruction theorem, Theorem 1.1 in the introduction.

Our task in the next two sections will be to show that under suitable additional assumptions these necessary conditions for the existence of an adapted wedge metric of psc are also sufficient.

Recall now that for these special pseudomanifolds we have defined a bordism theory $\Omega_{*}^{\text {spin, }(L, G)-\mathrm{fb}}(-)$; see Part I, Section 4. We end this section by observing that in this special case of $(L, G)$-fibered pseudomanifolds we can frame the alpha classes of this section in the following elegant way.

Proposition 3.1. Let $\Omega_{*}^{\mathrm{spin},(L, G)-\mathrm{fb}}(-)$ be the singular spin bordism group. Then we have welldefined homomorphisms:

$$
\begin{aligned}
& \alpha_{w}^{\Gamma}: \quad \Omega_{*}^{\mathrm{spin},(L, G)-\mathrm{fb}}(B \Gamma) \rightarrow K O_{*}\left(C_{r, \mathbb{R}}^{*}(\Gamma)\right), \\
& \alpha_{\text {cyl }}^{\Gamma}: \Omega_{*}^{\mathrm{spin},(L, G)-\mathrm{fb}}(B \Gamma) \rightarrow K O_{*}\left(C_{r, \mathbb{R}}^{*}(\Gamma)\right), \\
& \alpha_{\beta}^{\Gamma}: \Omega_{*}^{\mathrm{spin},(L, G)-\mathrm{fb}}(B \Gamma) \rightarrow K O_{*-\ell-1}\left(C_{r, \mathbb{R}}^{*}(\Gamma)\right) .
\end{aligned}
$$

Proof. Let $\left[M_{\Sigma}, f: M_{\Sigma} \rightarrow B \Gamma\right] \in \Omega_{*}^{\mathrm{spin},(L, G) \text {-fb }}(B \Gamma)$. We know that $(L, G)$-fibered pseudomanifolds are geometric-Witt. Fix an adapted wedge metric $g$ on $M_{\Sigma}$ and set

$$
\alpha_{w}^{\Gamma}\left(\left[M_{\Sigma}, f: M_{\Sigma} \rightarrow B \Gamma\right]\right):=\alpha_{w}^{\Gamma}\left(M_{\Sigma}, g, f\right) \in K O_{*}\left(C_{r, \mathbb{R}}^{*}(\Gamma)\right) .
$$

By definition, bordant elements in the group $\Omega_{*}^{\text {spin, }(L, G)-\mathrm{fb}}(B \Gamma)$ are in fact geometric-Witt bordant. Thus the first result follows immediately from Theorem 2.17. Regarding the cylindrical class, exactly the same arguments given for the $\alpha_{\text {cyl }}$ invariant in Part I, apply to $\alpha_{\text {cyl }}^{\Gamma}$, thus showing that

$$
\alpha_{\text {cyl }}^{\Gamma}: \Omega_{*}^{\text {spin },(L, G)-\mathrm{fb}}(B \Gamma) \rightarrow K O_{*}\left(C_{r, \mathbb{R}}^{*}(\Gamma)\right)
$$

is well defined.

Regarding (3.1): we have a homomorphism $\beta_{B \Gamma}: \Omega_{*}^{\text {spin, }(L, G)-\mathrm{fb}}(B \Gamma) \rightarrow \Omega_{*-\ell-1}(B \Gamma)$, associating to $\left[M_{\Sigma}, f: M_{\Sigma} \rightarrow B \Gamma\right]$ the class $\left[\beta M,\left.f\right|_{\beta M}: \beta M \rightarrow B \Gamma\right]$. The homomorphism (3.1) is obtained by composing this homomorphism $\beta_{B \Gamma}$ with the well-known alpha homomorphism for closed spin manifolds. Thus $\alpha_{\beta}^{\Gamma}\left[M_{\Sigma}, f: M_{\Sigma} \rightarrow B \Gamma\right]$ is equal to the $C_{r, \mathbb{R}}^{*}(\Gamma)$-index of the AtiyahSinger-Mishchenko operator $\mathfrak{P}_{\mathrm{MF}, g_{\beta M}}^{\beta}$ obtained by twisting $\mathfrak{P}_{g_{\beta M}}^{\beta}$ with the Mishchenko bundle associated to the $\Gamma$-cover $\Gamma \rightarrow \beta M^{\Gamma} \rightarrow \beta M$ defined by $\left.f\right|_{\beta M}: \beta M \rightarrow B \Gamma$; it is therefore well defined. 


\subsection{Fundamental groups and $K O$-obstructions for $(L, G)$-fibered pseudomanifolds}

We assume now that $\Gamma=\pi_{1}\left(M_{\Sigma}\right)$. We also consider $\pi_{1}(\beta M)$. In this section we want to be particularly precise about the discrete groups involved in the various Galois coverings.

- In all of this section we assume that $M_{\Sigma}$ has an adapted wedge metric $g$ of psc.

Under this assumption we observe that $\left(M, g_{M}\right)$ is of psc and we certainly have that

$$
\alpha_{w}^{\pi_{1}\left(M_{\Sigma}\right)}\left(M_{\Sigma}, g\right)=0=\alpha_{\mathrm{cyl}}^{\pi_{1}\left(M_{\Sigma}\right)}\left(M, g_{M}\right) \quad \text { in } \quad K O_{*}\left(C_{r, \mathbb{R}}^{*}\left(\pi_{1}\left(M_{\Sigma}\right)\right)\right)
$$

where the first class is relative to $\widetilde{M_{\Sigma}}$, the universal cover of $M_{\Sigma}$, and the second class is relative to $M^{\pi_{1}\left(M_{\Sigma}\right)}$, the restriction of $\widetilde{M_{\Sigma}}$ to the inverse image of $M$. Also, since $\beta M$ admits a metric of psc, we also have, as already remarked,

$$
\alpha^{\pi_{1}\left(M_{\Sigma}\right)}(\beta M)=0 \quad \text { in } \quad K O_{n-\ell-1}\left(C_{r, \mathbb{R}}^{*}\left(\pi_{1}\left(M_{\Sigma}\right)\right)\right),
$$

where this class is relative to the $\pi_{1}\left(M_{\Sigma}\right)$-cover of $\beta M$ obtained by taking the restriction of $\widetilde{M_{\Sigma}}$ to the inverse image of $\beta M$ (this is in fact the singular locus of $\widetilde{M_{\Sigma}}$ ). In addition we also have

$$
\alpha^{\pi_{1}(\beta M)}(\beta M)=0 \quad \text { in } \quad K O_{n-\ell-1}\left(C_{r, \mathbb{R}}^{*}\left(\pi_{1}(\beta M)\right)\right),
$$

where this class is relative to $\widetilde{\beta M}$, the universal cover of $\beta M, \pi_{1}(\beta M) \rightarrow \widetilde{\beta M} \rightarrow \beta M$, and it is defined in terms of $\mathfrak{P}_{\mathrm{MF}, \pi_{1}(\beta M)}$, the operator $\mathfrak{P}_{g_{\beta M}}^{\beta}$ twisted by the Mishchenko bundle

$$
\widetilde{\beta M} \times_{\pi_{1}(\beta M)} C_{r, \mathbb{R}}^{*}\left(\pi_{1}(\beta M)\right) .
$$

We remark that if $L$ is simply connected (for example a sphere, complex or quaternionic projective space, or complex Grassmannian), then the fibration $L \rightarrow \partial M \stackrel{\varphi}{\rightarrow} \beta M$ gives that $\varphi_{*}: \pi_{1}(\partial M) \rightarrow \pi_{1}(\beta M)$ is an isomorphism; moreover, the tubular neighborhood $N$ of $\beta M$ has a deformation retraction down to $\beta M$. An easy application of Van Kampen's theorem then proves that

$$
\pi_{1}\left(M_{\Sigma}\right) \simeq \pi_{1}(M)
$$

Thus in this case there are exactly two fundamental groups to keep track of, $\pi_{1}\left(M_{\Sigma}\right) \equiv \pi_{1}(M)$ and $\pi_{1}(\beta M)$.

We summarize the index obstructions when $L$ is 1-connected and we take into account the fundamental groups, in the following proposition.

Proposition 3.2. Let $M_{\Sigma}$ be a $(L, G)$-fibered pseudomanifold. Assume that $L$ is 1-connected so that $\pi_{1}\left(M_{\Sigma}\right) \equiv \pi_{1}(M)$. Let $g$ be an adapted wedge metric of psc on $M_{\Sigma}$. We endow $M$ with the metric $g_{M}:=\left.g\right|_{M}$. Under the above hypothesis we have the following vanishing results:

$$
\begin{array}{ll}
\alpha_{w}^{\pi_{1}\left(M_{\Sigma}\right)}\left(M_{\Sigma}, g\right)=\alpha_{\text {cyl }}^{\pi_{1}\left(M_{\Sigma}\right)}\left(M, g_{M}\right)=0 \quad \text { in } \quad K O_{n}\left(C_{\mathbb{R}, r}^{*}\left(\pi_{1}\left(M_{\Sigma}\right)\right)\right), \\
\alpha^{\pi_{1}\left(M_{\Sigma}\right)}(\beta M)=0 \quad \text { in } \quad K O_{n-\ell-1}\left(C_{\mathbb{R}, r}^{*}\left(\pi_{1}\left(M_{\Sigma}\right)\right)\right), \\
\alpha^{\pi_{1}(\beta M)}(\beta M)=0 \quad \text { in } \quad K O_{n-\ell-1}\left(C_{\mathbb{R}, r}^{*}\left(\pi_{1}(\beta M)\right)\right),
\end{array}
$$

where the first two classes are relative to $\widetilde{M_{\Sigma}}$, the universal cover of $M_{\Sigma}$, and to its restriction to the inverse image of $M$ respectively; the third class is relative to the restriction of $\widetilde{M_{\Sigma}}$ to the inverse image of $\beta M$, and the fourth class is defined in terms of $\widetilde{\beta M}$, the universal cover of $\beta M$. 


\section{Relevant surgery and bordism theorems}

\subsection{The case of a single fundamental group}

We need two simple generalizations of our results from Part I [14, Theorems 4.6 and 4.7]. Given a simply connected link manifold $L$ and a simply connected Lie group $G$, acting on $L$, we consider $(L, G)$-singular spin pseudomanifolds $M_{\Sigma}$, where $M_{\Sigma}=M \cup_{\partial M} N(\beta M)$, and the manifolds $M$ and $\beta M$ have non-trivial fundamental groups. The first case to analyze is when the pseudomanifold $M_{\Sigma}$ and its strata $M$ and $\beta M$ have the same fundamental group $\Gamma$. While this case is also covered by the more complicated case to be treated below in Section 4.2, we have separated this case out because the argument is simpler and clearer. In particular, such an $M_{\Sigma}$ determines an element in the bordism group $\Omega_{*}^{\text {spin, }(L, G)-\mathrm{fb}}(B \Gamma)$. In more detail, this means that there are maps $\xi: M_{\Sigma} \rightarrow B \Gamma$ and $\xi_{\beta}: \beta M \rightarrow B \Gamma$ such that the following diagram of isomorphisms is commutative:

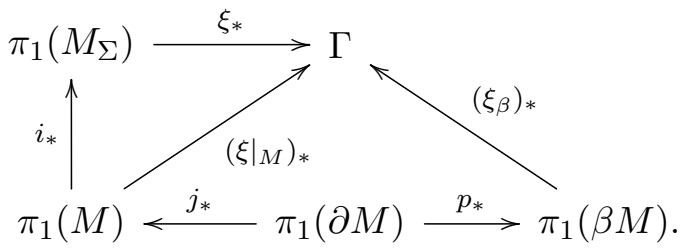

Here $i: M \hookrightarrow M_{\Sigma}, j: \partial M \hookrightarrow M$ are canonical embeddings.

Theorem 4.1 (a refined surgery theorem). Assume $G$ is a simply connected Lie group, $L$ is simply connected and spin, and $\Gamma$ is a discrete group. Let the element $x \in \Omega_{*}^{\mathrm{spin},(L, G)-\mathrm{fb}}(B \Gamma)$ be represented by two $(L, G)$-singular spin pseudomanifolds $\xi: M_{\Sigma} \rightarrow B \Gamma$ and $\xi^{\prime}: M_{\Sigma}^{\prime} \rightarrow B \Gamma$ of dimension $n \geq 6+\ell$, where $M_{\Sigma}=M \cup_{\partial M}-N(\beta M), M_{\Sigma}^{\prime}=M^{\prime} \cup_{\partial M}-N\left(\beta M^{\prime}\right)$ are given together with maps $\xi_{\beta}: \beta M \rightarrow B \Gamma$ and $\xi_{\beta}^{\prime}: \beta M^{\prime} \rightarrow B \Gamma$ and with structure maps $f: \beta M \rightarrow B G$ and $f^{\prime}: \beta M \rightarrow B G$. Assume the pseudomanifold $M_{\Sigma}$ and its strata $M$ and $\beta M$ have the same fundamental group $\Gamma$, i.e., (4.1) is a commutative diagram of isomorphisms.

Then there exists an $(L, G)$-bordism $W_{\Sigma}: M_{\Sigma} \rightsquigarrow M_{\Sigma}^{\prime}, W_{\Sigma}=W \cup_{\partial W} N(\beta W)$ over BГ, with a structure map $\bar{f}: \beta W \rightarrow B G$ restricting to the structure maps on $\beta M$ and $\beta M^{\prime}$, such that the pairs $(\beta W, \beta M)$ and $(W, M)$ are 2 -connected.

Remark 4.2. We emphasize that the maps $\xi^{\prime}: M_{\Sigma}^{\prime} \rightarrow B \Gamma$ and $\xi_{\beta}^{\prime}: \beta M^{\prime} \rightarrow B \Gamma$ are not assumed to be isomorphisms on fundamental groups; the only requirement here is that $\left(M_{\Sigma}^{\prime}, \xi^{\prime}, \xi_{\beta}^{\prime}\right)$ represents the same bordism class $x \in \Omega_{*}^{\mathrm{spin},(L, G)-\mathrm{fb}}(B \Gamma)$ as $\left(M_{\Sigma}, \xi, \xi_{\beta}\right)$.

Proof. We start with some $(L, G)$-bordism $W_{\Sigma}: M_{\Sigma} \rightsquigarrow M_{\Sigma}^{\prime}$ over $B \Gamma$. In particular, we have maps $\bar{\xi}: W_{\Sigma} \rightarrow B \Gamma$ and $\bar{\xi}_{\beta}: \beta W \rightarrow B \Gamma$ such that $\left.\bar{\xi}\right|_{M_{\Sigma}}=\xi$ and $\left.\bar{\xi}_{\beta}\right|_{\beta M_{\Sigma}}=\xi_{\beta}$.

Remark 4.3. For future use, we recall that $W_{\Sigma}=W \cup_{\partial^{(1)} W} N(\beta W)$, where $W$ is a manifold with corners: $\partial W=\partial^{(0)} W \cup \partial^{(1)} W$, where

$$
\partial^{(0)} W=M \sqcup-M^{\prime}, \quad \partial\left(\partial^{(0)} W\right)=-\partial\left(\partial^{(1)} W\right)=\partial M \sqcup-\partial M^{\prime},
$$

and the $(L, G)$-fiber bundle $\partial^{(1)} W$ is given by the map $\bar{f}: \beta W \rightarrow B G$, where $\left.\bar{f}\right|_{\beta M}=f$ and $\left.\bar{f}\right|_{\beta M^{\prime}}=f^{\prime}$. Then we denote by

$$
\delta W_{\Sigma}=\partial^{(0)} W \cup_{\partial\left(\partial^{(0)} W\right)} N(\partial(\beta W))=M_{\Sigma} \sqcup M_{\Sigma}^{\prime} .
$$


By assumption, the maps $\xi$ and $\xi_{\beta}$ induce isomorphisms of fundamental groups. As already remarked, the restrictions $\left.\bar{\xi}\right|_{M_{\Sigma}^{\prime}}=\xi^{\prime}$ and $\left.\bar{\xi}_{\beta}\right|_{\beta M_{\Sigma}^{\prime}}=\xi_{\beta}^{\prime}$ may not be isomorphisms on $\pi_{1}$. However, the commutativity of the diagrams

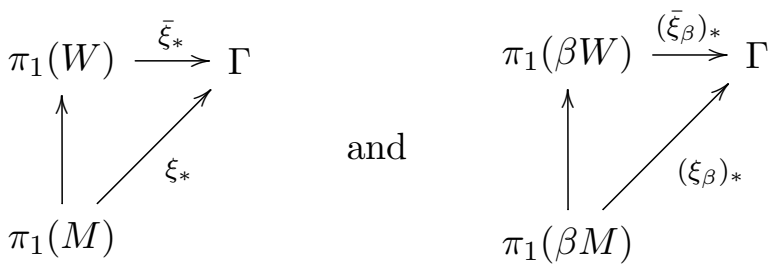

(with the vertical arrows induced by the inclusions) implies that the homomorphisms $\bar{\xi}_{*}$ : $\pi_{1}(W) \rightarrow \Gamma$ and $\left(\bar{\xi}_{\beta}\right)_{*}: \pi_{1}(\beta W) \rightarrow \Gamma$ are surjective.

Then the first step is to do surgery on $\beta W$ to make the pair $(\beta W, \beta M) 2$-connected. We begin by killing the kernel of $\pi_{1}(\beta W) \rightarrow \Gamma$. This proceeds as in the proof of [14, Theorem 4.7], the point being that since this kernel maps trivially to $\pi_{1}(B \Gamma)$, the surgery can be done over $B \Gamma$ without any extra effort. After taking care of the fundamental group, the situation is the same as in [14, Theorem 4.7]. The process for making the pair $(W, M)$ 2-connected is completely analogous.

Once we have established Theorem 4.1, we get a corresponding bordism theorem; see Theorem 4.6 from Part I.

Theorem 4.4 (a refined bordism theorem). Assume $G$ is a simply connected Lie group, $L$ is simply connected and spin, and $\Gamma$ is a discrete group. Let the element $x \in \Omega_{*}^{\mathrm{spin},(L, G)-\mathrm{fb}}(B \Gamma)$ be represented by two $(L, G)$-singular spin pseudomanifolds $\xi: M_{\Sigma} \rightarrow B \Gamma$ and $\xi^{\prime}: M_{\Sigma}^{\prime} \rightarrow B \Gamma$ of dimension $n \geq 6+\ell$, where $M_{\Sigma}=M \cup_{\partial M}-N(\beta M), M_{\Sigma}^{\prime}=M^{\prime} \cup_{\partial M}-N\left(\beta M^{\prime}\right)$ are given together with maps $\xi_{\beta}: \beta M \rightarrow B \Gamma$ and $\xi_{\beta}^{\prime}: \beta M^{\prime} \rightarrow B \Gamma$ and with structure maps $f: \beta M \rightarrow B G$ and $f^{\prime}: \beta M \rightarrow B G$. Assume the pseudomanifold $M_{\Sigma}$ and its strata $M$ and $\beta M$ have the same fundamental group $\Gamma$, i.e., (4.1) is a commutative diagram of isomorphisms. Furthermore, assume $M_{\Sigma}^{\prime}$ has an adapted psc metric $g^{\prime}$.

Then there exists an $(L, G)$-bordism $W_{\Sigma}: M_{\Sigma} \rightsquigarrow M_{\Sigma}^{\prime}$ over $B \Gamma$ together with an adapted psc metric $\bar{g}$ which is a product metric near the boundary $\delta W_{\Sigma}=M_{\Sigma} \sqcup-M_{\Sigma}^{\prime}$ such that $\left.\bar{g}\right|_{M_{\Sigma}^{\prime}}=g^{\prime}$. In particular, $M_{\Sigma}$ admits an adapted psc metric $g$.

\subsection{The case of two fundamental groups}

Next, we would like to address the natural situation when the fundamental groups of $\beta M$ and $M$ are different. For that, we need to redo some of the bordism theory of [14, Section 4] and fix two discrete groups $\Gamma_{\beta}$ and $\Gamma$ and a homomorphism $\theta: \Gamma_{\beta} \rightarrow \Gamma$. This data will be held fixed, and we consider pseudomanifolds $M_{\Sigma}=M \cup_{\partial M}-N(\beta M)$ with $(L, G)$-fibered singularities ${ }^{3}$ but now $\Gamma_{\beta}$ is the fundamental group of $\beta M$ and $\partial M$ and $\Gamma$ is the fundamental group of $M$, and $\theta: \Gamma_{\beta} \rightarrow \Gamma$ is the map of groups induced by the inclusion. To describe a bordism group $\Omega_{n}^{\text {spin, }(L, G)-\mathrm{fb}}\left(B \Gamma_{\beta} \stackrel{\theta_{*}}{\rightarrow} B \Gamma\right)$, we need maps $\xi: M \rightarrow B \Gamma$ and $\xi_{\beta}: \beta M \rightarrow B \Gamma_{\beta}$ such that the following diagram commutes:

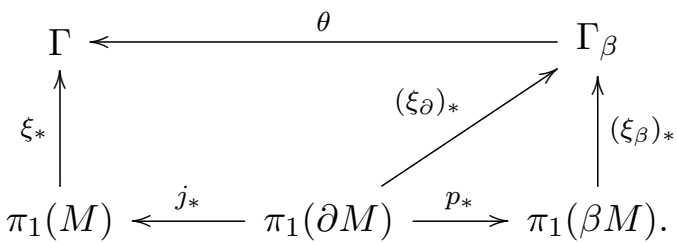

\footnotetext{
${ }^{3}$ Where as before $L$ and $G$ are simply connected and $G$ acts transitively on $L$.
} 
(Here $\xi_{\partial}=\xi_{\beta} \circ p$.) In the case of interest, since $L$ is simply connected, $p_{*}: \pi_{1}(\partial M) \rightarrow \pi_{1}(\beta M)$, $\xi_{*}: \pi_{1} M \rightarrow \Gamma$, and $\left(\xi_{\beta}\right)_{*}: \pi_{1}(\beta M) \rightarrow \Gamma_{\beta}$ are all isomorphisms. Notice that in our case, as already remarked, by Van Kampen's theorem, the fundamental group of $M_{\Sigma}$ is $\pi_{1}(M) *_{\pi_{1}(\beta M)} \pi_{1}(\beta M)=$ $\pi_{1}(M)=\Gamma$.

Definition 4.5. Fix a group homomorphism $\theta: \Gamma_{\beta} \rightarrow \Gamma$. We define $\Omega_{n}^{\operatorname{spin},(L, G)-\mathrm{fb}}\left(B \Gamma_{\beta} \stackrel{\theta_{*}}{\rightarrow} B \Gamma\right)$ to be the bordism group of $n$-dimensional closed $(L, G)$-singular spin pseudomanifolds $M_{\Sigma}$, where $M_{\Sigma}=M \cup_{\partial M}-N(\beta M)$ is equipped with maps $\xi: M \rightarrow B \Gamma$ and $\xi_{\beta}: \beta M \rightarrow B \Gamma_{\beta}$ such that the diagram

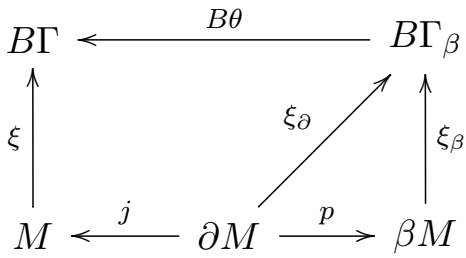

commutes up to homotopy (here $\xi_{\partial}=\xi_{\beta} \circ p$ ). A bordism

$$
\left(W_{\Sigma}, \bar{\xi}, \bar{\xi}_{\beta}\right):\left(M_{\Sigma}, \xi, \xi_{\beta}\right) \rightsquigarrow\left(M_{\Sigma}^{\prime}, \xi^{\prime}, \xi_{\beta}^{\prime}\right),
$$

between such objects is an $(L, G)$-singular spin pseudomanifold $W_{\Sigma}=W \cup_{\partial W} N(\beta W)$ with the boundary $\delta W_{\Sigma}=M_{\Sigma} \sqcup-M_{\Sigma}^{\prime}$ given together with maps $\bar{\xi}: W \rightarrow B \Gamma$ and $\bar{\xi}_{\beta}: \beta W \rightarrow B \Gamma_{\beta}$ such that

$$
\left.\bar{\xi}\right|_{M}=\xi,\left.\quad \bar{\xi}\right|_{M^{\prime}}=\xi^{\prime} ;\left.\quad \bar{\xi}_{\beta}\right|_{\beta M}=\xi_{\beta},\left.\quad \bar{\xi}_{\beta}\right|_{\beta M^{\prime}}=\xi_{\beta}^{\prime} .
$$

Remark 4.6. Note that, in general, $W$ here is a manifold with corners with $\partial W=\partial^{(0)} W \cup$ $\partial^{(1)} W$, where $\partial^{(0)} W \cap \partial^{(1)} W=\partial M \sqcup-\partial M^{\prime}$ and $\partial^{(1)} W$ has an $(L, G)$-fibration structure over $\beta W$, so that the map $\bar{\xi}_{\partial}$ is just the bundle projection followed by $\bar{\xi}_{\beta}$.

Remark 4.7. Together with structure maps $f: \beta M \rightarrow B G, f^{\prime}: \beta M^{\prime} \rightarrow B G$ and $\bar{f}: \beta W \rightarrow B G$, we obtain a spin bordism $\left(\bar{f} \times \bar{\xi}_{\beta}: \beta W \rightarrow B G \times B \Gamma_{\beta}\right)$ between $\left(f \times \xi_{\beta}: \beta M \rightarrow B G \times B \Gamma_{\beta}\right)$ and $\left(f^{\prime} \times \xi_{\beta}^{\prime}: \beta M^{\prime} \rightarrow B G \times B \Gamma_{\beta}\right)$.

Remark 4.8. Let $M_{\Sigma}=M \cup_{\partial M}-N(\beta M)$ be as above, i.e., $\pi_{1} M=\Gamma, \pi_{1} \partial M=\pi_{1} \beta M=\Gamma_{\beta}$, and the homomorphism $\theta: \Gamma_{\beta} \rightarrow \Gamma$ coincides with the one given by the inclusion $\partial M \hookrightarrow M$. Then there is a canonical map $u: M_{\Sigma} \rightarrow B \Gamma$ such that $\left.u\right|_{M}=\xi$, and $\left.u\right|_{N(\beta M)}$ is given by the composition $N(\beta M) \stackrel{\text { proj }}{\longrightarrow} \beta M \stackrel{\xi_{\beta}}{\longrightarrow} B \Gamma_{\beta} \stackrel{\theta}{\rightarrow} B \Gamma$. This construction defines a natural forgetful map

$$
\Omega_{n}^{\mathrm{spin},(L, G)-\mathrm{fb}}\left(B \Gamma_{\beta} \stackrel{B \theta}{\longrightarrow} B \Gamma\right) \rightarrow \Omega_{n}^{\mathrm{spin},(L, G)-\mathrm{fb}}(B \Gamma),
$$

which sends $\left(M_{\Sigma}, \xi, \xi_{\beta}\right)$ to $\left(M_{\Sigma}, u\right)$ as above.

To generalize the arguments in [14] for proving existence of well-adapted positive scalar curvature metrics in some cases, we now need an exact sequence into which the bordism group of Definition 4.5 will fit. In general this is quite complicated, so we only do an easy case as follows.

Theorem 4.9. Let $\Omega_{n}^{\mathrm{spin},(L, G)-\mathrm{fb}}\left(B \Gamma_{\beta} \stackrel{B \theta}{\longrightarrow} B \Gamma\right)$ be a bordism group as in Definition 4.5. Assume that $L$ is a $G$-equivariant spin boundary, i.e., that there is a spin $G$-manifold $\bar{L}$ with $\partial \bar{L}=L$ (as $G$-manifolds). Then there is an exact sequence of bordism groups

$$
0 \rightarrow \Omega_{n}^{\text {spin }}(B \Gamma) \stackrel{i}{\rightarrow} \Omega_{n}^{\text {spin },(L, G)-\mathrm{fb}}\left(B \Gamma_{\beta} \stackrel{B \theta}{\rightarrow} B \Gamma\right) \stackrel{\beta}{\rightarrow} \Omega_{n-\ell-1}^{\text {spin }}\left(B \Gamma_{\beta} \times B G\right) \rightarrow 0 .
$$


Proof. First of all, note that the assumption on $L$ gives the surjectivity of $\beta$. For if the map $\beta M \rightarrow B \Gamma_{\beta} \times B G$ represents an element of $\Omega_{n-\ell-1}^{\text {spin }}\left(B \Gamma_{\beta} \times B G\right)$ and we form the associated $(L, G)$-bundle

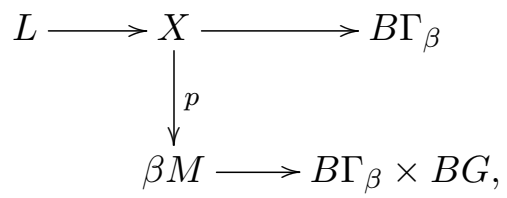

then by replacing $L$ by $\bar{L}$, we get a spin manifold $\bar{X}$ of dimension $n$ with boundary $X$, fibering over $\beta M$ with fiber $\bar{L}$ and fiber bundle projection $\bar{p}$. Now we have a commutative diagram

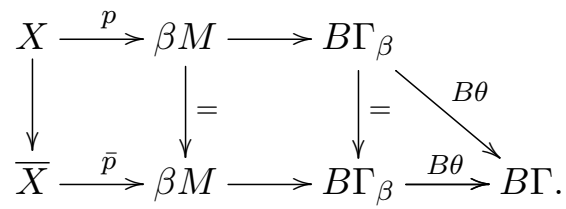

The result is a spin manifold $\bar{X}$ with boundary $X$, representing a class in

$$
\Omega_{n}^{\text {spin },(L, G)-\mathrm{fb}}\left(B \Gamma_{\beta} \stackrel{\theta}{\rightarrow} B \Gamma\right),
$$

mapping under $\beta$ to the class of $\beta M \rightarrow B \Gamma_{\beta} \times B G$.

The map $i: \Omega_{n}^{\text {spin }}(B \Gamma) \rightarrow \Omega_{n}^{\text {spin, }(L, G)-\mathrm{fb}}\left(B \Gamma_{\beta} \stackrel{\theta}{\rightarrow} B \Gamma\right)$ just comes from thinking of a closed manifold as a manifold with empty fibered singularities. We need to show this map is injective. Suppose $M \stackrel{\xi}{\rightarrow} B \Gamma$ represents a class in $\Omega_{n}^{\text {spin }}(B \Gamma)$. Suppose it bounds as a manifold with $(L, G)$ fibered singularities (in the bordism group in the center). Since $\partial M$ and $\beta M$ are empty, that means we have an $(L, G)$-singular spin pseudomanifold with boundary $W_{\Sigma}=W \cup_{\partial W} N(\beta W)$ with the boundary $\partial W_{\Sigma}=M$ and maps $\bar{\xi}: W \rightarrow B \Gamma, \bar{\xi}_{\partial}: \partial^{(1)} W \rightarrow B \Gamma_{\beta}$ and $\bar{\xi}_{\beta}: \beta W \rightarrow B \Gamma_{\beta}$ such that $\left.\bar{\xi}\right|_{M}=\xi$. Also, in this case, there are no corners, i.e., $X=\partial^{(1)} W$ is disjoint from $\partial^{(0)} W=M$. Now we want to modify $W$ to convert it to a manifold with boundary exactly equal to $M$. Once again, we use the assumption that $L$ is a spin $G$-boundary to build an $(\bar{L}, G)$-fiber bundle $\bar{X}$ over $\beta W$ bounding $X$, and $W \cup_{\partial^{(1)} W} \bar{X}$ is our required null-bordism of $M$. Thus the class of $M \stackrel{\xi}{\rightarrow} B \Gamma$ was already zero in $\Omega_{n}^{\text {spin }}(B \Gamma)$.

Finally, we need to prove exactness in the middle. Suppose given $M_{\Sigma}$ defining a class in

$$
\Omega_{n}^{\mathrm{spin},(L, G)-\mathrm{fb}}\left(B \Gamma_{\beta} \stackrel{B \theta}{\longrightarrow} B \Gamma\right)
$$

with $\left[\beta M \rightarrow B \Gamma_{\beta} \times B G\right]$ trivial in $\Omega_{n-\ell-1}^{\text {spin }}\left(B \Gamma_{\beta} \times B G\right)$. Choose a spin manifold $Y$ bounding $\beta M$, with a map to $B \Gamma_{\beta} \times B G$ extending the original map on $\beta M$. Form the associated $(L, G)$ fiber bundle $X$ over $Y$. Now by gluing $-X$ to $M$ along the common boundary $\partial M$ we get a closed spin $n$-manifold $M_{1}$ with a map to $B \Gamma$ coming from $M$. Indeed, this map extends over $-X$ via the composite $X \rightarrow B \Gamma_{\beta} \stackrel{B \theta}{\longrightarrow} B \Gamma$. Then $M_{1}$ represents the same class as $M_{\Sigma}$, because

$$
W=(M \times[0,1]) \cup_{\partial M \times\{1\}} X
$$

is a bordism between them in the sense of Definition 4.5.

Theorem 4.10 (surgery theorem, general version). Assume $G$ is a simply connected Lie group, $L^{\ell}$ is simply connected and spin, $\theta: \Gamma_{\beta} \rightarrow \Gamma$ is a group homomorphism, and $n \geq 6+\ell$. Let $x \in \Omega_{n}^{\mathrm{spin},(L, G)-\mathrm{fb}}\left(B \Gamma_{\beta} \stackrel{\theta_{*}}{\rightarrow} B \Gamma\right)$ be represented by two $(L, G)$-singular spin pseudomanifolds 
$\left(M_{\Sigma}, \xi, \xi_{\beta}\right), M_{\Sigma}=M \cup_{\partial M}-N(\beta M)$, and $\left(M_{\Sigma}^{\prime}, \xi^{\prime}, \xi_{\beta}^{\prime}\right), M_{\Sigma}^{\prime}=M^{\prime} \cup_{\partial M}-N\left(\beta M^{\prime}\right)$ given together with maps

$$
\xi_{\beta}: \beta M \rightarrow B \Gamma_{\beta}, \quad \text { and } \quad \xi_{\beta}^{\prime}: \beta M^{\prime} \rightarrow B \Gamma_{\beta},
$$

such that $\xi_{*}: \pi_{1} M \rightarrow \Gamma$ and $\left(\xi_{\beta}\right)_{*}: \pi_{1} \beta M \rightarrow \Gamma_{\beta}$ are isomorphisms. Then there exists an $(L, G)-$ bordism

$$
\left(W_{\Sigma}, \bar{\xi}, \bar{\xi}_{\beta}\right):\left(M_{\Sigma}, \xi, \xi_{\beta}\right) \rightsquigarrow\left(M_{\Sigma}^{\prime}, \xi^{\prime}, \xi_{\beta}^{\prime}\right),
$$

as in Definition 4.5 with the additional property that the pairs $(\beta W, \beta M)$ and $(W, M)$ are 2 -connected.

Proof. Start with an $(L, G)$-bordism $W_{\Sigma}: M_{\Sigma} \rightsquigarrow M_{\Sigma}^{\prime}$ in the sense of Definition 4.5. We have a commutative diagram

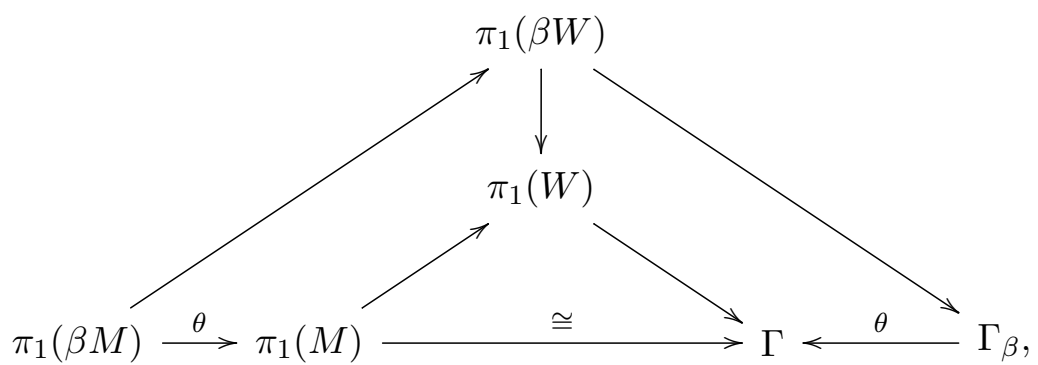

where the composite $\pi_{1}(\beta M) \rightarrow \pi_{1}(\beta W) \rightarrow \Gamma_{\beta}$ (along the top triangle) is an isomorphism, and thus the homomorphism $\pi_{1}(\beta W) \rightarrow \Gamma_{\beta}$ is surjective. The first step is to do surgery on $\beta W$ to make the pair $(\beta W, \beta M)$ 2-connected. We begin by killing the kernel of the surjection $\pi_{1}(\beta W) \rightarrow \Gamma_{\beta}$, just as in the proof of Theorem 4.1, so that the inclusion of $\beta M \hookrightarrow \beta W$ induces an isomorphism on $\pi_{1}$. Once this is done, we have an exact sequence

$$
\pi_{2}(\beta M) \rightarrow \pi_{2}(\beta W) \rightarrow \pi_{2}(\beta W, \beta M) \stackrel{0}{\rightarrow} \pi_{1}(\beta M) \stackrel{\cong}{\rightarrow} \pi_{1}(\beta W) .
$$

Then we need to do surgery on certain embedded 2-spheres in $\beta W$, generating $\pi_{2}(\beta W, \beta M)$. These embedded 2-spheres have trivial normal bundle because of the spin assumption, and map null-homotopically to the classifying space $B G$ (since the Lie group $G$ is simply connected, hence automatically 2 -connected $-\pi_{2}$ of any Lie group vanishes - and thus $B G$ is 3 -connected). So the necessary surgery is possible. The process for making the pair $(W, M) 2$-connected is completely analogous.

The following result is a straightforward generalization of known bordism results for psc metrics. Here Theorem 4.10 provides all the necessary tools.

Theorem 4.11 (bordism theorem, general version). Assume $G$ is a simply connected Lie group, $L^{\ell}$ is simply connected and spin, $\theta: \Gamma_{\beta} \rightarrow \Gamma$ is a group homomorphism, and $n \geq 6+\ell$. Let $x \in \Omega_{n}^{\mathrm{spin},(L, G)-\mathrm{fb}}\left(B \Gamma_{\beta} \stackrel{\theta_{*}}{\rightarrow} B \Gamma\right)$ be represented by two $(L, G)$-singular spin pseudomanifolds $\left(M_{\Sigma}, \xi, \xi_{\beta}\right), M_{\Sigma}=M \cup_{\partial M} N(\beta M)$, and $\left(M_{\Sigma}^{\prime}, \xi^{\prime}, \xi_{\beta}^{\prime}\right), M_{\Sigma}^{\prime}=M^{\prime} \cup_{\partial M} N\left(\beta M^{\prime}\right)$ given together with maps

$$
\xi: M \rightarrow B \Gamma, \quad \xi_{\beta}: \beta M \rightarrow B \Gamma_{\beta} \quad \text { and } \quad \xi^{\prime}: M^{\prime} \rightarrow B \Gamma, \quad \xi_{\beta}^{\prime}: \beta M^{\prime} \rightarrow B \Gamma_{\beta}
$$

such that $\xi_{*}: \pi_{1} M_{\Sigma} \rightarrow \Gamma$ and $\left(\xi_{\beta}\right)_{*}: \pi_{1} \beta M \rightarrow \Gamma_{\beta}$ are isomorphisms. Furthermore, assume $M_{\Sigma}^{\prime}$ has an adapted psc metric $g^{\prime}$. Then there exists an $(L, G)$-bordism

$$
\left(W_{\Sigma}, \bar{\xi}, \bar{\xi}_{\beta}\right):\left(M_{\Sigma}, \xi, \xi_{\beta}\right) \rightsquigarrow\left(M_{\Sigma}^{\prime}, \xi^{\prime}, \xi_{\beta}^{\prime}\right),
$$

together with an adapted psc metric $\bar{g}$ which is a product metric near the boundary $\delta W_{\Sigma}=$ $M_{\Sigma} \sqcup-M_{\Sigma}^{\prime}$ such that $\left.\bar{g}\right|_{M_{\Sigma}^{\prime}}=g^{\prime}$. In particular, $M_{\Sigma}$ admits an adapted psc metric $g$.

Proof. This proceeds like the proof Theorem 4.6 from Part I, using Theorem 4.10. 


\section{The existence theorem in the non-simply connected case}

In this section we will use the results from Section 4 to obtain existence theorems for welladapted positive scalar curvature metrics on singular pseudomanifolds in suitable cases. These results are parallel to those in [14, Section 6], but without the assumption that $M$ and $\partial M$ are simply connected. However, we will have to assume something about the fundamental groups. We denote by $\operatorname{Bott}^{8}$ a simply connected spin 8 -manifold with $\widehat{A}$-genus 1 , which one can take to be Ricci-flat [27]. This is often called a "Bott manifold", because taking the product with it in spin bordism corresponds, after applying the $\alpha$-transformation to $K O_{*}$, to the Bott periodicity map $K O_{*} \cong K O_{*+8}$. Let $\Gamma$ be a discrete group. We need the following definitions.

(GLR) A group $\Gamma$ satisfies the Gromov-Lawson-Rosenberg conjecture (GLR conjecture) if a closed connected spin manifold $M$ with dimension $n \geq 5$ and fundamental group $\Gamma$ admits a psc metric if and only if the generalized index $\alpha^{\Gamma}(M) \in K O_{n}\left(C_{r, \mathbb{R}}^{*}(\Gamma)\right)$ of the Dirac operator (i.e., the Atiyah-Singer-Mishchenko operator) on $M$ vanishes.

$\left({ }^{s}\right.$ GLR) A group $\Gamma$ satisfies the stable Gromov-Lawson-Rosenberg conjecture ( ${ }^{s}$ GLR conjecture) if for any closed connected spin manifold $M$ with fundamental group $\Gamma$, the vanishing of the generalized index $\alpha^{\Gamma}(M) \in K O_{n}\left(C_{r, \mathbb{R}}^{*}(\Gamma)\right)$ implies that $M \times\left(\mathrm{Bott}^{8}\right)^{k}$ admits a psc metric for some sufficiently large $k$.

We recall that Stolz ([45] and [46, Section 3]) sketched a proof that the ${ }^{s}$ GLR conjecture holds whenever the Baum-Connes assembly map is injective, which is true for a very large class of groups (conjecturally, all groups!). The GLR conjecture is satisfied for a more restricted class of groups, but including free groups, fundamental groups of oriented surfaces, and free abelian groups (as long as one takes $n$ bigger than the rank of the group). It also holds [13] for finite groups with periodic cohomology, which includes finite cyclic groups and quaternion groups. But it fails for some groups [20,43]. Our first main result is a generalization of [14, Theorem 6.3]. Recall the setting:

We fix a simply connected homogeneous space $L=G / H$, where $G$ is a compact semisimple Lie group acting on $L$ by isometries of the metric $g_{L}$, where $\operatorname{scal}_{g_{L}}=\mathrm{scal}_{S^{\ell}}=\ell(\ell-1)$, where $\ell=\operatorname{dim} L$.

Theorem 5.1. Let $M_{\Sigma}$ be an n-dimensional compact pseudomanifold with resolution $M$, a spin manifold with boundary $\partial M$. Assume the following:

(1) $M$ is a spin manifold with boundary $\partial M$ fibered over a connected spin manifold $\beta M$,

(2) the fiber bundle $\phi: \partial M \rightarrow \beta M$ is a geometric $(L, G)$-bundle.

Let $\Gamma=\pi_{1}(M), \Gamma_{\beta}=\pi_{1}(\beta M)$. Furthermore, assume $n \geq \ell+6$ and that the following condition holds:

- the link $L$ is a spin $G$-boundary of a $G$-manifold $\bar{L}$ equipped with a psc metric $g_{\bar{L}}$ which is a product near the boundary and satisfies $\left.g_{\bar{L}}\right|_{L}=g_{L}$.

Then, provided both groups $\Gamma$ and $\Gamma_{\beta}$ satisfy GLR conjecture, the vanishing of the invariants

$$
\alpha_{\text {cyl }}^{\Gamma}\left(M, g_{M}\right) \in K O_{n}\left(C_{r, \mathbb{R}}^{*}(\Gamma)\right) \quad \text { and } \quad \alpha^{\Gamma_{\beta}}(\beta M) \in K O_{n}\left(C_{r, \mathbb{R}}^{*}\left(\Gamma_{\beta}\right)\right)
$$

implies that $M_{\Sigma}$ admits an adapted psc metric.

Proof. Before we start with the proof we emphasize that under the assumptions above the index $\alpha_{\text {cyl }}^{\Gamma}(M)$ is well defined, independent of the chosen adapted wedge metric $g$; see Remark 2.9 
and Proposition 3.1. According to Theorems 4.11 and 4.9, it suffices to show that the bordism class $^{4}$

$$
\left[M_{\Sigma}\right] \in \Omega_{n}^{\mathrm{spin},(L, G)-\mathrm{fb}}\left(B \Gamma_{\beta} \stackrel{B \theta}{\longrightarrow} B \Gamma\right)
$$

is a sum of classes of pseudomanifolds with well-adapted positive scalar curvature metrics. We use the exact sequence (4.2). Since $\alpha^{\Gamma_{\beta}}(\beta M)=0$ and $\Gamma_{\beta}$ satisfies the GLR conjecture, and since $\operatorname{dim} \beta M=n-\ell-1 \geq 5$, the manifold $\beta M$ admits a psc metric. Then, since the fibration $\phi: \partial M \rightarrow \beta M$ is a geometric $(L, G)$-bundle; we can identify $\partial M$ with $P \times_{G} L$, where $P \rightarrow \beta M$ is the corresponding principal $G$-bundle. We use a bundle connection on $P$ to construct a well-adapted psc metric on the tubular neighborhood $N$ of $\beta M$ in $M_{\Sigma}$. In particular, we obtain a psc metric on the boundary $\partial N$, where the $(L, G)$-fiber bundle $\partial N \rightarrow \beta M$ is also a Riemannian submersion.

We use the $G$-manifold $\bar{L}$ which bounds $L$ to construct an $\bar{L}$-bundle $M^{\prime}=P \times_{G} \bar{L}$ over $\beta M$ associated to the above principal $G$-bundle $P \rightarrow \beta M$. By assumptions, $\bar{L}$ is given a psc metric $g_{\bar{L}}$ which is a product metric near $\partial \bar{L}=L$. Then $M^{\prime}$ has a bundle metric of positive scalar curvature, and joining $M^{\prime}$ to $N$, we get an $(L, G)$-singular spin manifold

$$
M_{\Sigma}^{\prime}=M^{\prime} \cup_{\partial M}-N
$$

with a well-adapted psc metric.

Since the pseudomanifolds $M_{\Sigma}^{\prime}$ and $M_{\Sigma}$ coincide near $\beta M$, by (4.2) their bordism classes differ by a class $\left[M^{\prime \prime}\right]$ in the image of $\Omega_{n}^{\text {spin }}(B \Gamma)$. Since, by assumption, we have $\alpha_{\text {cyl }}^{\Gamma}(M)=0$, and also $\alpha_{\text {cyl }}^{\Gamma}\left(M^{\prime}\right)=0$ (since $M^{\prime}$ has a psc metric), we have by additivity of the $\alpha$-invariant (Proposition 2.15) that $\alpha^{\Gamma}\left(M^{\prime \prime}\right)=0$, and so we can take $M^{\prime \prime}$ to be a closed spin $n$-manifold with fundamental group $\Gamma$ equipped with some psc metric. Now $M_{\Sigma}$ is in the same bordism class in

$$
\Omega_{n}^{\mathrm{spin},(L, G)-\mathrm{fb}}\left(B \Gamma_{\beta} \stackrel{B \theta}{\longrightarrow} B \Gamma\right)
$$

as $M_{\Sigma}^{\prime} \sqcup M^{\prime \prime}$, and we can apply Theorem 4.11 to get the conclusion.

Theorem 5.2. Let a link $L$ and pseudomanifold $M_{\Sigma}$ be as in Theorem 5.1, but this time only assume that the groups $\Gamma$ and $\Gamma_{\beta}$ satisfy the ${ }^{s} G L R$ conjecture. Then $M_{\Sigma}$ stably admits a well-adapted metric of positive scalar curvature, i.e., $M_{\Sigma} \times\left(\operatorname{Bott}^{8}\right)^{k}$ admits such a metric for some $k \geq 0$.

Proof. The proof of this is exactly the same as for Theorem 5.1, the only difference being that first we need to cross with some copies of the Bott manifold to get positive scalar curvature on $\beta M$, and then we might need to cross with additional copies of the Bott manifold to get positive scalar curvature on $M^{\prime \prime}$.

Remark 5.3. It is possible without great effort to adapt the arguments above to the case where $\beta M$ is disconnected. We leave details to the reader.

Now we present a generalization of $[14$, Theorem 6.7]. It was shown in [14, Lemma 6.6] that the class of $\mathbb{H}^{2 k}, k \geq 1$, is not a zero-divisor in the spin bordism ring $\Omega_{*}^{\text {spin }}$. It then follows that if $H_{*}(B \Gamma ; \mathbb{Z})$ is torsion free and the Atiyah-Hirzebruch spectral sequence converging to $\Omega_{*}^{\text {spin }}(B \Gamma)$ collapses, so that $\Omega_{*}^{\text {spin }}(B \Gamma)$ is a free $\Omega_{*}^{\text {spin }}$-module $\Omega_{*}^{\text {spin }} \otimes_{\mathbb{Z}} H_{*}(B \Gamma ; \mathbb{Z})$, then the class of $\mathbb{H}^{2 k}$ does not annihilate any non-zero class in $\Omega_{*}^{\text {spin }}(B \Gamma)$. This condition on $\Gamma$ is satisfied if $B \Gamma$ is stably homotopy-equivalent to a wedge of spheres - for example, if $\Gamma$ is a free group or free abelian group.

\footnotetext{
${ }^{4}$ Here we use a short notation $M_{\Sigma}$ instead of $\left(M_{\Sigma}, \xi, \xi_{\beta}\right)$.
} 
Theorem 5.4. Let $\left(M_{\Sigma}, \xi, \xi_{\beta}\right)$ be a closed $(L, G)$-singular spin pseudomanifold, $\operatorname{dim} M_{\Sigma}=n$, with $L=\mathbb{H} \mathbb{P}^{2 k}$ and $G=\operatorname{Sp}(2 k+1), k \geq 1$. Assume that

(i) the manifolds $M$ and $\beta M$ are connected and the inclusion $\partial M \hookrightarrow M$ induces an isomorphism on fundamental groups with $\pi_{1} M \cong \pi_{1} \partial M=\Gamma$,

(ii) the $(L, G)$-bundle $\partial M \rightarrow \beta M$ is trivial, i.e., $\partial M=\beta M \times L$,

(iii) the group $\Gamma$ satisfies the GLR conjecture,

(iv) $\Omega_{*}^{\text {spin }}(B \Gamma)$ is free as an $\Omega_{*}^{\text {spin }}$-module.

Then if $n-8 k \geq 6$, the pseudomanifold $M_{\Sigma}$ has an adapted psc metric if and only if the $\alpha$-invariant $\alpha_{\text {cyl }}^{\Gamma}(M) \in K O_{n}\left(C_{r, \mathbb{R}}^{*}(\Gamma)\right)$ vanishes.

Remark 5.5. Note that in this case we have isomorphisms $\pi_{1} M \cong \pi_{1} \partial M \cong \pi_{1} \beta M$. Also recall that if the bundle $\partial M \rightarrow \beta M$ is trivial, then the singularities are of Baas-Sullivan type.

Proof. Necessity was proved in Section 3, so we need to prove existence of an adapted metric of positive scalar curvature assuming the vanishing condition. Since $\partial M=\beta M \times L$ is a spin boundary over $B \Gamma$ (namely, it is the boundary of $M$ ), its class is trivial in $\Omega_{*}^{\text {spin }}(B \Gamma)$. But the class of $L$ cannot annihilate any non-zero class in $\Omega_{*}^{\text {spin }}(B \Gamma)$, by [14, Lemma 6.6], so in fact $\beta M$ must be a spin boundary over $B \Gamma$. (In particular, $\alpha^{\Gamma}(\beta M)$ automatically vanishes.) Now by [14, Proposition 4.4], or equivalently, by the proof of middle-exactness in the proof of Theorem 4.9 (this part of the proof doesn't require $L$ to be a spin boundary), $M_{\Sigma}$ is bordant over $B \Gamma$ to a closed manifold $M^{\prime}$ with fundamental group $\Gamma$ and no singularities. Then we must have $\alpha^{\Gamma}\left(M^{\prime}\right)=\alpha_{\text {cyl }}^{\Gamma}(M)=0$, and since $\Gamma$ satisfies the GLR conjecture, $M^{\prime}$ admits a metric of positive scalar curvature. The conclusion now follows from Theorem 4.4.

Theorem 5.6. Let $\left(M_{\Sigma}, \xi, \xi_{\beta}\right)$ be a closed $(L, G)$-singular spin pseudomanifold, $\operatorname{dim} M_{\Sigma}=n$, with $L=\mathbb{H} \mathbb{P}^{2 k}$ and $G=\operatorname{Sp}(2 k+1), k \geq 1$. Assume that

(i) the manifolds $M$ and $\beta M$ are connected and the inclusion $\partial M \hookrightarrow M$ induces an isomorphism on fundamental groups with $\pi_{1} M \cong \pi_{1} \partial M=\Gamma$,

(ii) the $(L, G)$-bundle $\partial M \rightarrow \beta M$ is trivial, i.e., $\partial M=\beta M \times L$,

(iii) the group $\Gamma$ satisfies the ${ }^{s} G L R$ conjecture,

(iv) $\Omega_{*}^{\text {spin }}(B \Gamma)$ is free as an $\Omega_{*}^{\text {spin }}$-module.

Then the pseudomanifold $M_{\Sigma} \times\left(\mathrm{Bott}^{8}\right)^{m}$ has an adapted metric of positive scalar curvature for some $m \geq 0$ if and only if the $\alpha$-invariant $\alpha_{\text {cyl }}^{\Gamma}(M) \in K O_{n}\left(C_{r, \mathbb{R}}^{*}(\Gamma)\right)$ vanishes.

Proof. This is exactly the same as the proof of Theorem 5.4, except that we need to multiply $M^{\prime}$ by some product of copies of the Bott manifold to get positive scalar curvature.

\section{The space $\mathcal{R}_{w}^{+}\left(M_{\Sigma}\right)$ of adapted wedge metrics of positive scalar curvature}

Let $M_{\Sigma}$ be a $(L, G)$-fibered pseudomanifold which admits a well-adapted wedge psc metric. In this section we shall study the space $\mathcal{R}_{w}^{+}\left(M_{\Sigma}\right)$ of well-adapted wedge psc metrics on $M_{\Sigma}$. Our first goal is to establish a relationship between the space $\mathcal{R}_{w}^{+}\left(M_{\Sigma}\right)$ and corresponding spaces $\mathcal{R}^{+}(\beta M), \mathcal{R}^{+}(\partial M), \mathcal{R}^{+}(M)$ of psc metrics. We consider the map ress $: \mathcal{R}_{w}^{+}\left(M_{\Sigma}\right) \rightarrow \mathcal{R}^{+}(\beta M)$ which takes an adapted wedge metric $g$ on $M_{\Sigma}$ to its restriction $g_{\beta M}$ on $\beta M$, and we will observe that this map is a Serre fiber bundle. We show (this was stated before as Theorem 1.5) that there exists a non-canonical section $s: \mathcal{R}^{+}(\beta M) \rightarrow \mathcal{R}_{w}^{+}\left(M_{\Sigma}\right)$; this fact, together with recent 
results on the homotopy type of the space of psc metrics on spin manifolds, [11, 24], implies that the homotopy groups of the space $\mathcal{R}_{w}^{+}\left(M_{\Sigma}\right)$ are rationally at least as complicated as the groups $K O_{*}\left(C_{r}^{*}\left(\pi_{1}(\beta M)\right)\right) \otimes \mathbb{Q}$. Next, we elaborate on a direct definition of the index-difference homomorphism which enables us to detect some nontrivial classes in $\pi_{q}\left(\mathcal{R}_{w}^{+}\left(M_{\Sigma}\right)\right)$. Finally, if the fundamental group of $M_{\Sigma}$ has an element of finite order, we show that the Cheeger-Gromov rho invariant on a depth-1 pseudomanifold, introduced and studied in [39], can be used to show that $\left|\pi_{0}\left(\mathcal{R}_{w}^{+}\left(M_{\Sigma}\right)\right)\right|=\infty$.

\subsection{Controlling the homotopy type of $\mathcal{R}_{w}^{+}\left(M_{\Sigma}\right)$}

We fix an $(L, G)$-fibered compact pseudomanifold $M_{\Sigma}=M \cup_{\partial M} N(\beta M)$. Here $M$ is a compact manifold with boundary $\partial M$ which is a geometric $(L, G)$-bundle over $\beta M$. Recall that the $G$ acts on $L$ by isometries of a given metric $g_{L}$, and the $(L, G)$-bundle $\phi: \partial M \rightarrow \beta M$ is given by a principal $G$-bundle $p: P \rightarrow \beta M$ so that $\partial M=P \times_{G} L$. Then we identify the space $\mathcal{R}_{w}^{+}\left(M_{\Sigma}\right)$ of well-adapted wedge psc metrics on $M_{\Sigma}$ with the space of associated triples $\left(g_{M}, g_{\beta M}, \nabla^{p}\right)$, where $g_{M}$ and $g_{\beta M}$ are psc metrics on $M$ and $\beta M$ respectively, and $\nabla^{p}$ is a connection on the principal bundle $P$. By definition of well-adapted wedge metrics, the triples $\left(g_{M}, g_{\beta M}, \nabla^{p}\right)$ are subject to the following conditions:

(i) $g_{M}=\mathrm{d} t^{2}+g_{\partial}$ near the boundary $\partial M \subset M$, for some Riemannian metric $g_{\partial}$ on $\partial M$;

(ii) the bundle map $\phi$ gives a Riemannian submersion $\phi:\left(\partial M, g_{\partial}\right) \rightarrow\left(\beta M, g_{\beta M}\right)$, and the connection $\nabla^{p}$ on $P$ defines a $G$-connection $\nabla^{\phi}$ for the submersion.

We denote by $\mathcal{R}^{+}(M, \partial M)$ the space of Riemannian psc metrics $g$ on $M$ such that $g=\mathrm{d} t^{2}+g_{\partial}$ near the boundary $\partial M$, for some metric $g_{\partial}$ on $\partial M$. There is an obvious restriction map

$$
\text { res: } \mathcal{R}^{+}(M, \partial M) \rightarrow \mathcal{R}^{+}(\partial M)
$$

which is known to be a Serre fiber bundle, see [23, Theorem 1.1]. For a given metric $h \in \mathcal{R}^{+}(\partial M)$, we denote by $\mathcal{R}^{+}(M)_{h}=\operatorname{res}^{-1}(h)$ the corresponding fiber of (6.1).

We notice that there is a map $\iota_{L}: \mathcal{R}^{+}(\beta M) \rightarrow \mathcal{R}^{+}(\partial M)$ which is given by lifting a metric $g_{\beta M}$ on $\beta M$ to a Riemannian submersion metric $g_{\partial M}$ on the total space $\partial M$ of the $(L, G)$-bundle $\phi: \partial M \rightarrow \beta M$, using the connection $\nabla^{\phi}$ of (ii) above, by putting the metric $g_{L}$ on each fiber $L$. This map is injective since the metrics $g_{\beta M}$ and $\iota_{L}\left(g_{\beta M}\right)$ determine one another. Then, by definition, a wedge psc metric $g$ on $M_{\Sigma}$ determines a unique psc metric $g_{\beta M} \in \mathcal{R}^{+}(\beta M)$. This gives a well-defined map $\operatorname{res}_{\Sigma}: \mathcal{R}_{w}^{+}\left(M_{\Sigma}\right) \rightarrow \mathcal{R}^{+}(\beta M)$. Thus we see that the space $\mathcal{R}_{w}^{+}\left(M_{\Sigma}\right)$ is a pull-back in the following diagram:

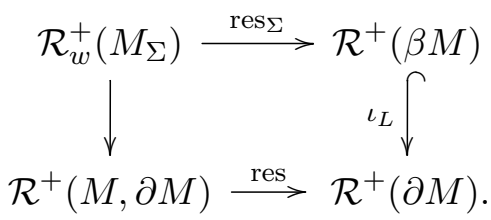

As we mentioned above, the restriction map res: $\mathcal{R}^{+}(M, \partial M) \rightarrow \mathcal{R}^{+}(\partial M)$ is a Serre fiber bundle, and it is easy to see that the map $\operatorname{res}_{\Sigma}: \mathcal{R}_{w}^{+}\left(M_{\Sigma}\right) \rightarrow \mathcal{R}^{+}(\beta M)$ from (6.2) is also a Serre fiber bundle. ${ }^{5}$ Let $g_{\beta M} \in \mathcal{R}^{+}(\beta M)$ and $g_{\partial M} \in \mathcal{R}^{+}(\partial M)$ be such that $\iota_{L}\left(g_{\beta M}\right)=g_{\partial M}$. We have the

\footnotetext{
${ }^{5}$ This requires a word of explanation, as follows: by [14, Theorem 3.5(2)], given a metric $g_{\beta} \in \mathcal{R}^{+}(\beta M)$ such that $\iota_{L}\left(g_{\beta}\right)$ extends to a metric in $\mathcal{R}^{+}(M, \partial M)$, then some rescaling of $g_{\beta}$ (by a constant conformal factor) lifts to $\mathcal{R}_{w}^{+}\left(M_{\Sigma}\right)$.
} 
following commutative diagram of fiber bundles:

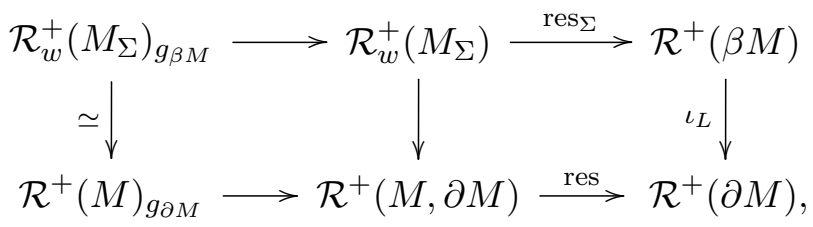

where $\mathcal{R}_{w}^{+}\left(M_{\Sigma}\right)_{g_{\beta M}}$ and $\mathcal{R}^{+}(M)_{g_{\partial M}}$ are the corresponding fibers. Note that the downward map on the left is a homotopy equivalence, in fact a homeomorphism, since any element of $\mathcal{R}^{+}(M)_{g_{\partial M}}$ defines a unique psc wedge metric.

In particular, if we have an isotopy $\left(g_{\beta M}\right)_{t} \in \mathcal{R}^{+}(\beta M)$, and $\left(g_{\beta M}\right)_{0}=\operatorname{res}_{\Sigma}\left(g_{0}\right)$, then the isotopy $\left(g_{\beta M}\right)_{t}$ lifts to an isotopy $g_{t}$ in $\mathcal{R}_{w}^{+}\left(M_{\Sigma}\right)$ with $\operatorname{res}_{\Sigma}\left(g_{t}\right)=\left(g_{\beta M}\right)_{t}$.

Theorem 6.1. Let $M_{\Sigma}$ be an $(L, G)$-fibered compact pseudomanifold with $L$ a simply connected homogeneous space of a compact semisimple Lie group. Also assume that $M_{\Sigma}$ admits an adapted wedge metric of positive scalar curvature. Then there exists a section $s: \mathcal{R}^{+}(\beta M) \rightarrow \mathcal{R}_{w}^{+}\left(M_{\Sigma}\right)$ to ress. In particular, there is an injection of homotopy groups

$$
s_{*}: \pi_{q}\left(\mathcal{R}^{+}(\beta M)\right) \rightarrow \pi_{q}\left(\mathcal{R}_{w}^{+}\left(M_{\Sigma}\right)\right), \quad q=0,1, \ldots
$$

Proof. We want to construct a (non-canonical) section to the "forgetful map"

$$
\operatorname{res}_{\Sigma}: \mathcal{R}_{w}^{+}\left(M_{\Sigma}\right) \rightarrow \mathcal{R}^{+}(\beta M)
$$

sending a psc metric $g$ (interpreted as a triple $\left(g_{M}, g_{\beta M}, \nabla^{p}\right)$, as above) to the metric $g_{\beta M}$. As before, we will fix the connection $\nabla^{p}$ once and for all, and we fix a metric $g_{\beta M} \in \mathcal{R}^{+}(\beta M)$. Given another metric $g_{\beta M}^{\prime}$ in $\mathcal{R}^{+}(\beta M)$, we consider the linear homotopy $g_{\beta M}(t)=(1-t) g_{\beta M}+$ $t g_{\beta M}^{\prime}$ within the space $\mathcal{R}(\beta M)$ of all Riemannian metrics. This homotopy may go out of the subspace $\mathcal{R}^{+}(\beta M)$, but the scalar curvature function along the homotopy is bounded below by some constant $-c, c>0$. If we scale the metrics $g_{\beta M}(t)$ by $\lambda^{2}$, then the scalar curvature scales by scal $\lambda_{\lambda^{2} g_{\beta M}(t)}=\lambda^{-2}$ scal $_{g_{\beta M}(t)}$. We consider the family of metrics $g_{\partial M}(t)=\iota_{L}\left(\lambda^{2} g_{\beta M}(t)\right)$ on $\partial M$. Recall that $\operatorname{scal}_{L}=\ell(\ell-1)>0$, so we obtain

$$
\operatorname{scal}_{g_{\partial M}(t)}=\operatorname{scal}_{g_{L}}+\lambda^{-2} \operatorname{scal}_{g_{\beta M}(t)} \geq \ell(\ell-1)-\lambda^{-2} c .
$$

Clearly, there exists $\lambda>0$ such that $\operatorname{scal}_{g_{\partial M}(t)}>0$ for all $t>0$. Then we lift the curve of metrics $g_{\partial M}(t)$ to a curve of metrics $g(t) \in \mathcal{R}^{+}(M, \partial M)$ using homotopy lifting in the fiber bundle

$$
\text { res: } \mathcal{R}^{+}(M, \partial M) \rightarrow \mathcal{R}^{+}(\partial M)
$$

In particular, we obtain an adapted wedge metric $\left(g_{M}^{\prime}, g_{\beta M}^{\prime}, \nabla^{p}\right) \in \mathcal{R}_{w}^{+}\left(M_{\Sigma}\right)$. It is important to notice the following:

1. The curve of the adapted wedge metrics $\left(g_{M}(t), g_{\beta M}(t), \nabla^{p}\right)$ is not, in general, a curve in the space $\mathcal{R}_{w}^{+}\left(M_{\Sigma}\right)$. Indeed, the scalar curvature on the tubular neighborhood $N(\beta M)$ is dominated by the sum of zero scalar curvature on the cone over $L$ and by the scalar curvature $\lambda^{-2} \operatorname{scal}_{g_{\beta M}(t)}{ }^{6}$

\footnotetext{
${ }^{6}$ An important comment: to apply the argument of [14, Theorem 3.5(2)] in order to rescale a metric $g_{\beta M}^{\prime}$ in $\mathcal{R}^{+}(\beta M)$ so that $\iota_{L}\left(g_{\beta M}^{\prime}\right)$ extends to a psc metric on the whole tubular neighborhood $N$ of $\beta M$, one needs to make use of a positive lower bound on the scalar curvature. So while $g_{\beta M}^{\prime}$ lifts to $\mathcal{R}_{w}^{+}\left(M_{\Sigma}\right.$ ) (after rescaling by a positive constant), the same is not true for the other metrics in the path in $\mathcal{R}(\beta M)$ from $g_{\beta M}$ to $g_{\beta M}^{\prime}$.
} 
2. The correspondence $g_{\beta M}^{\prime} \mapsto\left(g_{M}^{\prime}, g_{\beta M}^{\prime}, \nabla^{p}\right)$ can be made continuous (in the $C^{2}$ topology) as long as the metrics $g_{\beta M}^{\prime}$ stay inside a compact set $K \subset \mathcal{R}^{+}(\beta M)$.

This is enough to get a (non-canonical) section $s_{g_{\beta M}}: \mathcal{R}^{+}(\beta M) \rightarrow \mathcal{R}_{w}^{+}\left(M_{\Sigma}\right)$ of the fiber bundle $\operatorname{res}_{\Sigma}$ in $(6.2)$.

Corollary 6.2. The fiber bundle (6.3) gives a split short exact sequence:

$$
\begin{aligned}
& 0 \rightarrow \pi_{q}\left(\mathcal{R}_{w}^{+}\left(M_{\Sigma}\right)_{g_{\beta M}}\right) \stackrel{i_{*}}{\longrightarrow} \pi_{q}\left(\mathcal{R}_{w}^{+}\left(M_{\Sigma}\right)\right) \stackrel{\left(\operatorname{res}_{\Sigma}\right)_{*}}{\longrightarrow} \pi_{q}\left(\mathcal{R}^{+}(\beta M)\right) \rightarrow 0 \\
& \quad \text { for each } \quad q=0,1, \ldots
\end{aligned}
$$

Proof. Indeed, a section $s_{g_{\beta M}}: \mathcal{R}^{+}(\beta M) \rightarrow \mathcal{R}_{w}^{+}\left(M_{\Sigma}\right)$ from Theorem 6.1 implies that the homomorphism $\left(\operatorname{res}_{\Sigma}\right)_{*}$ is split surjective for all $q=0,1, \ldots$.

An immediate consequence of this corollary is that $\pi_{q}\left(\mathcal{R}_{w}^{+}\left(M_{\Sigma}\right)\right)$ admits $\pi_{q}\left(\mathcal{R}^{+}(\beta M)\right)$ as a direct summand. In particular, $\pi_{0}\left(\mathcal{R}_{w}^{+}\left(M_{\Sigma}\right)\right)$ contains $\pi_{0}\left(\mathcal{R}^{+}(\beta M)\right)$ as a direct summand.

Example 6.3. Here is an interesting example of obtaining information about $\pi_{0}\left(\mathcal{R}^{+}\left(M_{\Sigma}\right)\right)$. Let $L=G=\mathrm{SU}(2)=S^{3}$. Since $\pi_{7}\left(B S^{3}\right)=\pi_{6}\left(S^{3}\right)=\mathbb{Z} / 12$, there are 12 distinct principal $S^{3}$ bundles over $\beta M=S^{7}$, all of them of the rational homotopy type of $S^{7} \times S^{3}$. For any one of these $S^{3}$-bundles over $S^{7}$, the total space is $\partial M=\partial N$, where $M=N$ is the disk bundle of our $S^{3}$ bundle over $S^{7}$. Define $M_{\Sigma}=N \cup_{\partial M}-N$, the double of $N$. Note that $N$ is actually the unit disk bundle of a quaternionic line bundle, so our $M_{\Sigma}$ in this case is in fact a smooth manifold, though not every Riemannian metric on $M_{\Sigma}$ is adapted for the $(L, G)$-fibered structure, and we are only interested in this special subclass of metrics. We know that $\pi_{0}\left(\mathcal{R}_{w}^{+}\left(M_{\Sigma}\right)\right)$ contains $\pi_{0}\left(\mathcal{R}^{+}(\beta M)\right)=\pi_{0}\left(\mathcal{R}^{+}\left(S^{7}\right)\right)$ as a direct summand. By [26, Theorem 4.47], $\pi_{0}\left(\mathcal{R}^{+}\left(S^{7}\right)\right)$ contains a copy of $\mathbb{Z}$, and the same argument shows $\pi_{0}\left(\mathcal{R}^{+}\left(S^{11}\right)\right)$ contains a copy of $\mathbb{Z}$. By taking a connected sum of $\left(M_{\Sigma}, g_{\Sigma}\right)$ with $\left(S^{11}, g_{k}\right)$ for suitable metrics $g_{k}$ on $S^{11}$ (the connected sum takes place on the interior of $M$, so it doesn't change the adapted wedge structure of the metric near $\left.\beta M=S^{7}\right)$, we see that $\pi_{0}\left(\mathcal{R}_{w}^{+}\left(M_{\Sigma}\right)\right)$ contains $\mathbb{Z} \oplus \mathbb{Z}$, with one summand coming from $\beta M$ and one coming from the interior of $M$.

Remark 6.4. We recall that for a closed manifold $X$, the homotopy type of the space $\mathcal{R}^{+}(X)$ does not change under admissible surgeries; in particular, this implies that the homotopy type of $\mathcal{R}^{+}(X)$ depends only on the bordism class of $X$ in a relevant bordism group [23, Theorem 1.5]. As we have seen, on an $(L, G)$-pseudomanifold $M_{\Sigma}$, we can do two types of surgeries: surgeries on the interior of the resolution $M$, and surgeries on $\beta M$. It turns out that the homotopy type of the space $\mathcal{R}_{w}^{+}\left(M_{\Sigma}\right)$ is also invariant with respect to corresponding admissible surgeries, [16].

There are several methods for detecting non-trivial elements in $\pi_{q}\left(\mathcal{R}^{+}(\beta M)\right)$ : the indexdifference homomorphisms and (higher) rho-invariants are certainly two very efficient tools in this direction. In the Sections 6.2 we shall elaborate further on these two tools. Notice that some of the results proven by rho-invariants can also be recovered using the index-difference homomorphisms (6.5) explained below; see [24, Remark 1.1.2].

\subsection{The index-difference homomorphism}

First we recall some results from $[11,24]$. Let $\bar{X}$ be a compact closed spin manifold, $\operatorname{dim} \bar{X}=$ $k \geq 5$. Let $\bar{h}_{0} \in \mathcal{R}^{+}(\bar{X})$ be a base point. Assume there is a map $f: \bar{X} \rightarrow B \Gamma$, where $\Gamma$ is a discrete group such that $f_{*}: \pi_{1} \bar{X} \rightarrow \Gamma$ is split surjective. (If $\pi_{1} \bar{X}$ is trivial, we assume $\Gamma=0$.)

We also need the case when the boundary $\partial \bar{X}=X$ is non-empty, with a given metric $h \in \mathcal{R}^{+}(X)$. As before, we denote by $\mathcal{R}^{+}(\bar{X})_{h}$ the subspace of metrics $\bar{h}$ in $\mathcal{R}^{+}(\bar{X}, X)$ which 
restrict to $h$ on the boundary $X$. Let $\bar{h}_{0} \in \mathcal{R}^{+}(\bar{X})_{h}$ be a base point. Again, we assume that there is a map $f: \bar{X} \rightarrow B \Gamma$, such that $f_{*}: \pi_{1} \bar{X} \rightarrow \Gamma$ is split surjective. In both these settings (without using split surjectivity of $f_{*}$ yet) we have the index-difference homomorphisms

$$
\begin{aligned}
& \operatorname{inddiff} \bar{h}_{\bar{h}_{0}}^{\Gamma}: \pi_{q} \mathcal{R}^{+}(\bar{X}) \rightarrow K O_{q+k+1}\left(C_{r}^{*}(\Gamma)\right), \\
& \operatorname{inddiff} \bar{h}_{0}^{\Gamma}: \pi_{q} \mathcal{R}^{+}(\bar{X})_{h} \rightarrow K O_{q+k+1}\left(C_{r}^{*}(\Gamma)\right) \text {. }
\end{aligned}
$$

Since it will be useful later, we remind the reader about where these index difference maps come from. Say for simplicity that $\bar{X}$ is a closed manifold. A class in $\pi_{q} \mathcal{R}^{+}(\bar{X})$ is represented by a family $g_{t}, t \in S^{q}$, of psc metrics on $\bar{X}$, with $g_{t_{0}}, t_{0}$ the basepoint in $S^{q}$, equal to our basepoint psc metric. Let us denote here by $g_{0}$ this base-point metric. From this family we get a warped product metric $g$ on $S^{q} \times \bar{X}$, that restricts on the copy of $\bar{X}$ over $t \in S^{q}$ to $g_{t}$, and that is the usual flat (if $q \leq 1$ ) or round (if $q \geq 2$ ) metric on the copy of $S^{q}$ over each point in $\bar{X}$. By the usual argument that "isotopy implies concordance" [25, Lemma 3], we can, without changing the homotopy class of our map $t \mapsto g_{t}$ in $\pi_{q} \mathcal{R}^{+}(\bar{X})$, assume that $g$ has positive scalar curvature on $S^{q} \times \bar{X}$. Our class in $\pi_{q} \mathcal{R}^{+}(\bar{X})$ is trivial if and only if it can be extended over the disk $D^{q+1}$. So extend $g$ to a metric on $D^{q+1} \times \bar{X}$ which restricts to a product metric $\mathrm{d} r^{2}+g$ on a neighborhood of the boundary. Extend it to a complete metric on $\mathbb{R}^{q+1} \times \bar{X}$ by allowing $r$, the distance to the center of the disk, to go to $\infty$, and taking the metric to be $\mathrm{d} r^{2}+g$ for all $r \geq 1$. Since the metric has psc outside a compact set, the Dirac operator on $\mathbb{R}^{q+1} \times \bar{X}$ for this metric is Fredholm, and we get an index in $K O_{q+1+k}\left(C_{r}^{*}(\Gamma)\right)$ as usual. One way to see this property is to use $b$-calculus techniques; this point of view will be useful later on in this section. This index is an obstruction to triviality of our class in $\pi_{q} \mathcal{R}^{+}(\bar{X})$, since it would be 0 if we could extend our family of metrics to a psc family over the disk. Summarizing, we have defined a homomorphism

$$
\operatorname{inddiff}_{g_{0}}^{\Gamma}: \pi_{q} \mathcal{R}^{+}(\bar{X}) \rightarrow K O_{q+k+1}\left(C_{r}^{*}(\Gamma)\right) .
$$

An alternative way to construct the index difference is the following. Once we choose a base point $g_{0}=g_{t_{0}} \in \mathcal{R}^{+}(\bar{X})$, then for any metric $g \in \mathcal{R}^{+}(\bar{X})$, there is a linear path $g_{t}=(1-t) g_{0}+t g$ of metrics in the space $\mathcal{R}(\bar{X})$ of all Riemannian metrics. Then we have a curve of the corresponding Dirac operators $D_{g_{t}}$ with the ends of the curve, $D_{g_{0}}$ and $D_{g}$ being in the contractible space of $C \ell_{k}$-linear invertible operators. Such a curve determines a loop in the space $\mathbf{K} \mathbf{O}_{k}$ which classifies $C \ell_{k}$-linear Fredholm operators (where the space of invertible operators is collapsed to a point). One can easily replace this by the classifying space $\mathbf{K O}_{k}\left(C_{r}^{*}(\Gamma)\right)$ for the $K$-theory $K O_{*+k}\left(C_{r}^{*}(\Gamma)\right)$ in the non-simply connected case. Thus in homotopy we get the index-difference homomorphism

$$
\operatorname{inddiff}_{g_{0}}^{\Gamma}: \pi_{q} \mathcal{R}^{+}(\bar{X}) \rightarrow \pi_{q}\left(\Omega \mathbf{K O}_{k}\left(C_{r}^{*}(\Gamma)\right)\right)=K O_{q+1+k}\left(C_{r}^{*}(\Gamma)\right),
$$

which depends on a choice of the base point $g_{0} \in \mathcal{R}^{+}(\bar{X})$. The equivalence of these two approaches to the index difference (with a discussion of how to trace them back to the work of Gromov-Lawson and Hitchin, respectively) may be found in [21, 22] (see also [17]).

Next we recall the following relevant results:

Theorem 6.5 (see $[11,24]$ ).

(a) Let $\bar{X}$ be a simply connected spin manifold, with $\partial \bar{X}$ possibly non-empty, $\operatorname{dim} \bar{X}=k \geq 6$, with $\bar{h}_{0} \in \mathcal{R}^{+}(\bar{X}) \neq \varnothing\left(\right.$ or $\left.\bar{h}_{0} \in \mathcal{R}^{+}(\bar{X})_{h} \neq \varnothing\right)$. Then both index-difference homomorphisms inddiff $\Gamma_{\bar{h}_{0}}^{\Gamma}$ from (6.5) are non-trivial whenever the target group $\mathrm{KO}_{q+k+1}$ is.

(b) Let $\bar{X}$ be a non-simply connected spin manifold, with $\partial \bar{X}$ possibly non-empty, $\operatorname{dim} X=k \geq$ 6 with $\bar{h}_{0} \in \mathcal{R}^{+}(\bar{X}) \neq \varnothing\left(\right.$ or $\left.\bar{h}_{0} \in \mathcal{R}^{+}(\bar{X})_{h} \neq \varnothing\right)$. Assume there is a map $f: \bar{X} \rightarrow B \Gamma$, such that $f_{*}: \pi_{1} \bar{X} \rightarrow \Gamma$ is split surjective. Furthermore, we assume that 
- $\Gamma$ satisfies the rational Baum-Connes conjecture,

- $\Gamma$ is torsion free and has finite rational homological dimension d, and

- $q>d-2 k-1$.

Then the images of both index-difference homomorphisms inddiff $\bar{h}_{0}^{\Gamma}$ from (6.5) generate the target group $K O_{q+k+1}\left(C_{r}^{*}(\Gamma)\right) \otimes \mathbb{Q}$ as a $\mathbb{Q}$-vector space.

Recall also the following stable result. As in the beginning of Section 5, we denote by Bott ${ }^{8}$ a Bott manifold, which is a simply connected spin 8-manifold with $\hat{A}($ Bott $)=1$. By the work of Joyce [27], we may choose a Ricci flat metric $g_{b}$ on Bott. Then for a closed manifold $\bar{X}$ there are induced maps

$$
\mathcal{R}^{+}(\bar{X}) \stackrel{\times\left(\text { Bott }, g_{b}\right)}{\longrightarrow} \mathcal{R}^{+}(\bar{X} \times \text { Bott }) \stackrel{\times\left(\text { Bott }, g_{b}\right)}{\longrightarrow} \mathcal{R}^{+}(\bar{X} \times \text { Bott } \times \text { Bott }) \stackrel{\times\left(\text { Bott }, g_{b}\right)}{\longrightarrow} \cdots,
$$

and write $\mathcal{R}^{+}(\bar{X})\left[\mathrm{Bott}^{-1}\right]$ for the homotopy colimit. Similarly, if $\partial \bar{X}=X \neq \varnothing$, and $h \in \mathcal{R}^{+}(X)$, we define the space $\mathcal{R}^{+}(\bar{X})_{h}\left[\right.$ Bott $\left.^{-1}\right]$. Assume we have a reference map $f: \bar{X} \rightarrow B \Gamma$ which is surjective on the fundamental groups. Then the index-difference homomorphisms inddiff $\bar{h}_{0}$ extend to the corresponding index-difference homomorphisms

$$
\begin{aligned}
& \operatorname{inddiff} \bar{h}_{\bar{h}_{0}}^{\Gamma}: \pi_{q} \mathcal{R}^{+}(\bar{X})\left[\mathrm{Bott}^{-1}\right] \rightarrow K O_{q+k+1}\left(C_{r}^{*}(\Gamma)\right), \\
& \operatorname{inddiff} \bar{h}_{\bar{h}_{0}}^{\Gamma}: \pi_{q} \mathcal{R}^{+}(\bar{X})_{h}\left[\mathrm{Bott}^{-1}\right] \rightarrow K O_{q+k+1}\left(C_{r}^{*}(\Gamma)\right) .
\end{aligned}
$$

Here is one more relevant result:

Theorem 6.6 (see [24, Theorem B] and [17]). If $\Gamma$ is a torsion-free group satisfying the BaumConnes conjecture, and $f: \bar{X} \rightarrow B \Gamma$ is split surjective on the fundamental groups, then the homomorphisms (6.6) are surjective for all $q \geq 0$.

Now we are ready to apply those results to the case of $(L, G)$-pseudomanifolds. Let $M_{\Sigma}$ be an $(L, G)$-fibered compact pseudomanifold. We notice that if the space $\mathcal{R}_{w}^{+}\left(M_{\Sigma}\right)$ is not empty and $g_{0} \in \mathcal{R}_{w}^{+}\left(M_{\Sigma}\right)$ is a base point, then it determines base points in the corresponding spaces: the metrics $g_{\beta M, 0} \in \mathcal{R}^{+}(\beta M), g_{\partial M, 0} \in \mathcal{R}^{+}(\partial M)$ and $g_{M, 0} \in \mathcal{R}^{+}(M)_{g_{\partial M, 0}}$.

As we noticed above the spaces $\mathcal{R}_{w}^{+}\left(M_{\Sigma}\right)_{g_{\beta M, 0}}$ and $\mathcal{R}^{+}(M)_{g_{\partial M, 0}}$ from (6.3) are homotopy equivalent and the groups $\pi_{q}\left(\mathcal{R}_{w}^{+}\left(M_{\Sigma}\right)_{g_{\beta M, 0}}\right)$ in the exact sequence (6.4) could be replaced by $\pi_{q}\left(\mathcal{R}^{+}(M)_{g_{\partial M, 0}}\right)$. Thus we obtain the homomorphism

$$
\operatorname{inddiff}_{g_{M, 0}}: \pi_{q} \mathcal{R}_{w}^{+}\left(M_{\Sigma}\right)_{g_{\beta M, 0}} \stackrel{\cong}{\rightarrow} \pi_{q}\left(\mathcal{R}^{+}(M)_{g_{\partial M, 0}}\right) \stackrel{\text { inddiff }_{g_{\partial M, 0}}}{\longrightarrow} K O_{q+n+1},
$$

which, together with the index-difference homomorphism

$$
\operatorname{indiff}_{g_{\beta M, 0}}: \pi_{q}\left(\mathcal{R}^{+}(\beta M)\right) \rightarrow K O_{q+n-\ell},
$$

determines the homomorphism

$$
\operatorname{inddiff}_{g_{0}}: \pi_{q}\left(\mathcal{R}_{w}^{+}\left(M_{\Sigma}\right)\right) \stackrel{\text { inddiff }_{g_{\partial M, 0}} \oplus \text { inddiff }_{g_{\beta M, 0}}}{\longrightarrow} K O_{q+n+1} \oplus K O_{q+n-\ell} .
$$

Corollary 6.7. Let $M_{\Sigma}$ be a $(L, G)$-fibered compact pseudomanifold with $L$ a simply connected homogeneous space of a compact semisimple Lie group, and $n-\ell-1 \geq 5$, where $\operatorname{dim} M=n$, $\operatorname{dim} L=\ell$. Let $g_{0} \in \mathcal{R}_{w}^{+}\left(M_{\Sigma}\right) \neq \varnothing$ be a base point giving corresponding base points, the metrics $g_{\beta M, 0} \in \mathcal{R}^{+}(\beta M), g_{\partial M, 0} \in \mathcal{R}^{+}(\partial M)$ and $g_{M, 0} \in \mathcal{R}^{+}(M)_{g_{\partial M, 0}}$. 
If $M_{\Sigma}$ is spin and simply connected, then we have the following commutative diagram:

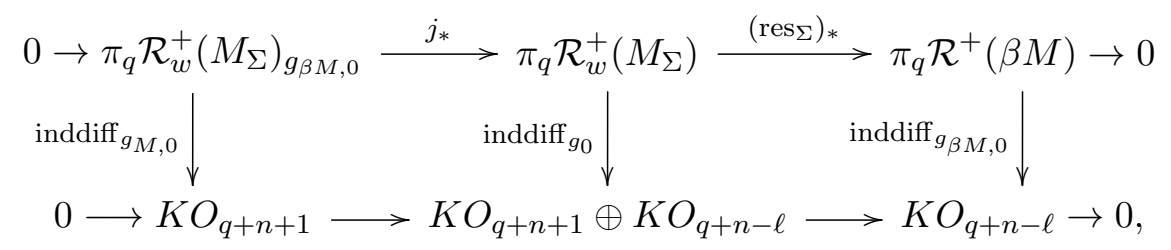

where the homomorphisms inddiff $g_{M, 0}$ and inddiff $g_{\beta M, 0}$ are both nontrivial whenever the target groups are. In particular, the homomorphism

$$
\text { inddiff } g_{0}: \pi_{q} \mathcal{R}_{w}^{+}\left(M_{\Sigma}\right) \rightarrow K O_{q+n+1} \oplus K O_{q+n-\ell}
$$

is surjective rationally and surjective onto the torsion of $K O_{q+n+1} \oplus K O_{q+n-\ell}$.

Proof. We use Corollary 6.2 to choose a splitting

$$
\pi_{q}\left(\mathcal{R}_{w}^{+}\left(M_{\Sigma}\right)\right) \cong \pi_{q}\left(\mathcal{R}_{w}^{+}\left(M_{\Sigma}\right)_{g_{\beta M}}\right) \oplus \pi_{q}\left(\mathcal{R}^{+}(\beta M)\right) \cong \pi_{q}\left(\mathcal{R}^{+}(M)_{g_{\partial M, 0}}\right) \oplus \pi_{q}\left(\mathcal{R}^{+}(\beta M)\right) .
$$

Then we can construct the homomorphism inddiff $g_{0}$ as a direct sum

$$
\text { inddiff }_{g_{0}}=\text { inddiff }_{g_{M, 0}} \oplus \text { inddiff }_{g_{\beta M, 0}}: \pi_{q} \mathcal{R}_{w}^{+}\left(M_{\Sigma}\right) \rightarrow K O_{q+n+1} \oplus K O_{q+n-\ell} .
$$

By Theorem 6.5(a), the individual index difference maps in this decomposition are non-trivial whenever the target $K O_{k}$ is non-zero. Since $K O_{k}=\mathbb{Z} / 2$ whenever it has torsion, that implies that the map surjects onto the torsion. Similarly, the part of Theorem 6.5(a) about the rational groups implies rational surjectivity.

Now we address the case when $M_{\Sigma}$ is not simply-connected. Let $\theta: \Gamma_{\beta} \rightarrow \Gamma$ be a group homomorphism as in Section 4. We consider a triple $\left(M_{\Sigma}, \xi, \xi_{\beta}\right)$ as an object representing an element in the bordism group $\Omega_{n}^{\text {spin, }(L, G) \text {-fb }}\left(B \Gamma_{\beta} \stackrel{\theta}{\rightarrow} B \Gamma\right)$, i.e., $M_{\Sigma}=M \cup_{\partial M} N(\beta M)$ comes together with the maps $\xi: M \rightarrow B \Gamma, \xi_{\beta}: \beta M \rightarrow B \Gamma_{\beta}$ satisfying the conditions given in Definition 4.5.

Thus we obtain the homomorphism

$$
\operatorname{inddiff}_{g_{M, 0}}^{\Gamma}: \pi_{q} \mathcal{R}_{w}^{+}\left(M_{\Sigma}\right)_{g_{\beta M, 0}} \stackrel{\cong}{\rightarrow} \pi_{q}\left(\mathcal{R}^{+}(M)_{g_{\partial M, 0}}\right) \stackrel{\text { indiff }_{g_{\partial M, 0}}}{\longrightarrow} K O_{q+n+1}\left(C_{r}^{*}(\Gamma)\right),
$$

which, together with the index-difference homomorphism

$$
\operatorname{inddiff}_{g_{\beta M, 0}}^{\Gamma_{\beta}}: \pi_{q}\left(\mathcal{R}^{+}(\beta M)\right) \rightarrow K O_{q+n-\ell}\left(C_{r}^{*}\left(\Gamma_{\beta}\right)\right),
$$

determines the homomorphism

$$
\operatorname{inddiff}_{g_{0}}^{\Gamma, \Gamma_{\beta}}: \pi_{q}\left(\mathcal{R}_{w}^{+}\left(M_{\Sigma}\right)\right) \stackrel{\text { inddiff }_{g_{\partial M, 0}} \oplus \text { inddiff }_{g_{\beta M, 0}}}{\longrightarrow} K O_{q+n+1}\left(C_{r}^{*}(\Gamma)\right) \oplus K O_{q+n-\ell}\left(C_{r}^{*}\left(\Gamma_{\beta}\right)\right) .
$$

The same argument as above and Theorem $6.5(b)$ prove the following:

Corollary 6.8. Let $M_{\Sigma}$ be a $(L, G)$-fibered compact pseudomanifold with $L$ a simply connected homogeneous space of a compact semisimple Lie group, and $n-\ell-1 \geq 5$, where $\operatorname{dim} M=n$, $\operatorname{dim} L=\ell$. Let $g_{0} \in \mathcal{R}_{w}^{+}\left(M_{\Sigma}\right) \neq \varnothing$ be a base point giving corresponding base points, the metrics $g_{\beta M, 0} \in \mathcal{R}^{+}(\beta M), g_{\partial M, 0} \in \mathcal{R}^{+}(\partial M)$ and $g_{M, 0} \in \mathcal{R}^{+}(M)_{g_{\partial M, 0}}$. Let $\left(M_{\Sigma}, \xi, \xi_{\beta}\right)$ represent an element in the bordism group $\Omega_{n}^{\mathrm{spin},(L, G)-\mathrm{fb}}\left(B \Gamma_{\beta} \stackrel{\theta}{\rightarrow} B \Gamma\right)$, then we have the following commutative diagram:

$$
\begin{gathered}
0 \rightarrow \pi_{q} \mathcal{R}_{w}^{+}\left(M_{\Sigma}\right)_{g_{\beta M, 0}} \longrightarrow \pi_{q} \mathcal{R}_{w}^{+}\left(M_{\Sigma}\right) \stackrel{\left(\operatorname{res}_{\Sigma}\right)_{*}}{\longrightarrow} \pi_{q} \mathcal{R}^{+}(\beta M) \rightarrow 0 \\
\quad \operatorname{inddiff}_{g_{0}}^{\Gamma, \Gamma_{\beta}} \downarrow \\
0 \longrightarrow K O_{q+n+1}\left(C_{r}^{*}(\Gamma)\right) \rightarrow K O_{q+n+1}\left(C_{r}^{*}(\Gamma)\right) \oplus K O_{q+n-\ell}\left(C_{r}^{*}\left(\Gamma_{\beta}\right)\right) \rightarrow K O_{q+n-\ell}\left(C_{r}^{*}\left(\Gamma_{\beta}\right)\right) \rightarrow 0 .
\end{gathered}
$$


If, in addition, the pair $\left(\Gamma, \Gamma_{\beta}\right)$ is such that

- $\Gamma$ and $\Gamma_{\beta}$ both satisfy the rational Baum-Connes conjecture,

- $\Gamma$ and $\Gamma_{\beta}$ are both torsion free and have finite rational homological dimension d, and

- $q>d-2 n+2 \ell+1$,

then the image of the homomorphism

$$
\operatorname{inddiff~}_{g_{0}}^{\Gamma, \Gamma_{\beta}}: \pi_{q} \mathcal{R}_{w}^{+}\left(M_{\Sigma}\right) \rightarrow K O_{q+n+1}\left(C_{r}^{*}(\Gamma)\right) \oplus K O_{q+n-\ell}\left(\left(C_{r}^{*}\left(\Gamma_{\beta}\right)\right)\right.
$$

generates the target $K O_{q+n+1}\left(C_{r}^{*}(\Gamma)\right) \oplus K O_{q+n-\ell}\left(\left(C_{r}^{*}\left(\Gamma_{\beta}\right)\right)\right) \otimes \mathbb{Q}$ as a $\mathbb{Q}$-vector space.

Let $\mathcal{R}^{+}\left(M_{\Sigma}\right)\left[\right.$ Bott $\left.^{-1}\right]$ be the homotopy colimit

$$
\mathcal{R}^{+}\left(M_{\Sigma}\right) \stackrel{\times\left(\mathrm{Bott}, g_{b}\right)}{\longrightarrow} \mathcal{R}^{+}\left(M_{\Sigma} \times \text { Bott }\right) \stackrel{\times\left(\text { Bott }, g_{b}\right)}{\longrightarrow} \mathcal{R}^{+}\left(M_{\Sigma} \times \text { Bott } \times \text { Bott }\right) \stackrel{\times\left(\text { Bott }, g_{b}\right)}{\longrightarrow} \cdots .
$$

We have the following conclusion from Theorems 6.6 and 6.1:

Corollary 6.9. Let $M_{\Sigma}$ be a $(L, G)$-fibered compact pseudomanifold with $L$ a simply connected homogeneous space of a compact semisimple Lie group, and $n-\ell-1 \geq 5$, where $\operatorname{dim} M=n$, $\operatorname{dim} L=\ell$. Let $g_{0} \in \mathcal{R}_{w}^{+}\left(M_{\Sigma}\right) \neq \varnothing$ be a base point giving corresponding base points, the metrics $g_{\beta M, 0} \in \mathcal{R}^{+}(\beta M), g_{\partial M, 0} \in \mathcal{R}^{+}(\partial M)$ and $g_{M, 0} \in \mathcal{R}^{+}(M)_{g_{\partial M, 0}}$. Let $\left(M_{\Sigma}, \xi, \xi_{\beta}\right)$ represent an element in the bordism group $\Omega_{n}^{\mathrm{spin},(L, G)-\mathrm{fb}}\left(B \Gamma_{\beta} \stackrel{\theta}{\rightarrow} B \Gamma\right)$. Assume that the pair $\left(\Gamma, \Gamma_{\beta}\right)$ is such that

- $\Gamma$ and $\Gamma_{\beta}$ both satisfy the Baum-Connes conjecture,

- $\Gamma$ and $\Gamma_{\beta}$ are both torsion free.

Then the homomorphism

$$
\operatorname{inddiff} g_{g_{0}}^{\Gamma, \Gamma_{\beta}}: \pi_{q} \mathcal{R}_{w}^{+}\left(M_{\Sigma}\right)\left[\mathrm{Bott}^{-1}\right] \rightarrow K O_{q+n+1}\left(C_{r}^{*}(\Gamma)\right) \oplus K O_{q+n-\ell}\left(\left(C_{r}^{*}\left(\Gamma_{\beta}\right)\right)\right.
$$

is surjective.

Example 6.10. In [24, Section 1.1.3], the authors provided an example of a group $\pi_{0}$ such that $K O_{7+k}\left(C_{r}^{*}\left(\pi_{0}\right)\right) \otimes \mathbb{Q}$ has countably infinite dimension for each $k \geq 0,{ }^{7}$ as well as a 4 dimensional closed spin manifold $X$ with $\pi_{1} X=\pi_{0}$. Then, according to [24, Theorem C], the manifold $Y=X \times S^{2}$ has the property that the group $\pi_{7+k} \mathcal{R}^{+}(Y) \otimes \mathbb{Q}$ has countably infinite dimension for each $k \geq 0$. Then it is easy to construct a pseudomanifold $M_{\Sigma}$ with $L=G=\mathrm{SU}(2)$ and $\beta M=Y$. Indeed, we let $M=X \times D^{3} \times L, \partial M=X \times S^{2} \times L$, $\beta M=X \times S^{2}$ and $M_{\Sigma}=M \cup_{\partial M}(Y \times c(L))$. Then, clearly, Theorem 6.1 and Corollary 6.8 imply that $\pi_{7+k} \mathcal{R}_{w}^{+}\left(M_{\Sigma}\right) \otimes \mathbb{Q}$ has countably infinite dimension for each $k \geq 0$.

\subsection{A direct approach to the index-difference homomorphism for $(L, G)$-fibered pseudomanifolds}

Let $M_{\Sigma}$ be as above, thus a $(L, G)$-fibered pseudomanifold, and let $g_{0}, g_{1} \in \mathcal{R}_{w}^{+}\left(M_{\Sigma}\right)$ be two well adapted wedge psc metrics. Then the "difference" between $g_{0}$ and $g_{1}$ could be detected directly by a wedge relative index as follows. We consider $M_{\Sigma} \times[0,1]$ as a pseudomanifold with boundary, and equip it with a well adapted wedge metric $\bar{g}$ of product-type near the boundary and equal

\footnotetext{
${ }^{7}$ Let $\mathrm{Free}_{2}$ be a free group on two generators. Then the group $\pi_{0}$ is a free product $\mathrm{SB}_{3} * \mathrm{SB}_{4} * \mathrm{SB}_{5} * \mathrm{SB}_{6}$, where $\mathrm{SB}_{r}$ is the $r$-th Stallings-Bieri group, the kernel of the homomorphism (Free $)^{r} \rightarrow \mathbb{Z}$ sending each generator to 1.
} 
to $g_{0}$ on $M_{\Sigma} \times\{0\}$ and to $g_{1}$ on $M_{\Sigma} \times\{1\}$. Now we attach infinite cylinders to $M_{\Sigma} \times[0,1]$ to get a wedge metric on the associated manifold with cylindrical ends $\left(M_{\Sigma} \times[0,1]\right)_{\infty}$ (which in this case is nothing but $M_{\Sigma} \times \mathbb{R}$ ), with psc metrics along the two ends. This is a special case of the situation already encountered in Section 2.4, see in particular Theorem 2.17. In particular, since the resulting metric on $M_{\Sigma} \times \mathbb{R}$ has psc along the cylindrical ends and is geometricWitt on the compact part, there is a well defined class $\alpha_{w}^{\mathrm{rel}}\left(g_{0}, g_{1}\right)$ in $K O_{n+1}$. This class, by definition, is equal to the cylindrical $\alpha_{w}$-class of the Atiyah-Singer operator on the manifold with cylindrical ends $\left(M_{\Sigma} \times[0,1]\right)_{\infty} \equiv M_{\Sigma} \times \mathbb{R}$ endowed with the natural extension of the metric $\bar{g}$ to a metric $\bar{g}_{\infty}$. The existence of this class follows immediately, as in Theorem 2.17, from the $b$-edge calculus developed in [1].

If the psc metrics $g_{0}$ and $g_{1}$ are isotopic, i.e., they lie in the same component of $\mathcal{R}_{w}^{+}\left(M_{\Sigma}\right)$, then the usual "isotopy $\Rightarrow$ concordance" argument shows that we can construct a psc metric $\bar{g}$ on $M_{\Sigma} \times[0,1]$, which implies that the metric $g_{\infty}$ is of psc. Hence the element $\alpha_{w}^{\mathrm{rel}}\left(g_{0}, g_{1}\right)$ in $K O_{n+1}$ gives an obstruction to an isotopy from $g_{0}$ to $g_{1}$ within $\mathcal{R}_{w}^{+}\left(M_{\Sigma}\right)$. Proceeding in the same way, but using the Atiyah-Singer-Mishchenko operator instead, we also have a class

$$
\alpha_{w}^{\mathrm{rel}, \pi_{1}\left(M_{\Sigma}\right)}\left(g_{0}, g_{1}\right) \in K O_{n+1}\left(C_{r, \mathbb{R}}^{*}\left(\pi_{1}\left(M_{\Sigma}\right)\right)\right)
$$

which is again an obstruction to the existence of an isotopy between $g_{0}$ and $g_{1}$.

More generally, for an $(L, G)$-fibered pseudomanifold we can adapt the general discussion given just before Theorem 6.5 and, using in a crucial way the results explained in Section 2, define directly the wedge-index-difference homomorphism

$$
w \text {-inddiff } \bar{h}_{0}^{\Gamma}: \pi_{q} \mathcal{R}_{w}^{+}\left(M_{\Sigma}\right) \rightarrow K O_{q+k+1}\left(C_{r}^{*}(\Gamma)\right)
$$

either by considering $S^{q} \times M_{\Sigma}$ and $D^{q} \times M_{\Sigma}$ or by employing the classifying space $\mathbf{K O}_{k}\left(C_{r}^{*}(\Gamma)\right)$.

It would be interesting to show, but we shall leave this to future research, that $w$-inddiff $\Gamma_{\bar{h}_{0}}$ is equal to the index-difference homomorphism considered in the previous section, that is, the composition of the isomorphism $\pi_{q}\left(\mathcal{R}_{w}^{+}\left(M_{\Sigma}\right)_{g_{\beta M, 0}}\right) \rightarrow \pi_{q}\left(\mathcal{R}^{+}(M)_{g_{\partial M, 0}}\right)$ with the indexdifference homomorphism for the manifold with boundary $M$, inddiff $\Gamma_{\partial M, 0}^{\Gamma}: \pi_{q}\left(\mathcal{R}^{+}(M)_{g_{\partial M, 0}}\right) \rightarrow$ $K O_{q+n+1}\left(C_{r}^{*} \Gamma\right)$.

\subsection{Rho invariants and torsion fundamental groups}

We begin by introducing the relevant bordism groups:

$$
\operatorname{Pos}_{n}^{\text {spin, },(L, G)-\mathrm{fb}}(B \Gamma) \quad \text { and } \quad \operatorname{Pos}_{n}^{\text {spin, }(L, G)-\mathrm{fb}}\left(B \Gamma_{\beta} \stackrel{B \theta}{\longrightarrow} B \Gamma\right) .
$$

We only give the definition of the latter of these two in detail, since $\operatorname{Pos}_{n}^{\operatorname{spin},(L, G)-\mathrm{fb}}(B \Gamma)$ is just a special case of $\operatorname{Pos}_{n}^{\text {spin, }(L, G)-\mathrm{fb}}\left(B \Gamma_{\beta} \stackrel{B \theta}{\longrightarrow} B \Gamma\right)$ when $\Gamma=\Gamma_{\beta}$ and $\theta$ is the identity map. Recall that an element of the bordism group $\Omega_{n}^{\text {spin, }(L, G)-\mathrm{fb}}\left(B \Gamma_{\beta} \stackrel{B \theta}{\longrightarrow} B \Gamma\right)$ is represented by a geometric cycle, i.e., a tuple

$$
\left(M, P \stackrel{p}{\rightarrow} \beta M, \xi: M \rightarrow B \Gamma, \xi_{\beta}: \beta M \rightarrow B \Gamma_{\beta}\right)
$$

with the same compatibility conditions for $\xi, B \theta, \xi_{\beta}$ as in Definition 4.5 and with $P \stackrel{p}{\rightarrow} \beta M$ a principal $G$-bundle such that $\partial M=P \times_{G} L$.

Similarly, the bordism relation in Definition 4.5 can be described as follows: two geometric cycles

$$
\left(M, P \stackrel{p}{\rightarrow} \beta M, \xi, \xi_{\beta}\right) \quad \text { and } \quad\left(M^{\prime}, P^{\prime} \stackrel{p^{\prime}}{\rightarrow} \beta M^{\prime}, \xi^{\prime}, \xi_{\beta}^{\prime}\right)
$$


are equivalent if there exists a manifold $W$ with corners such that $\partial W=\partial^{(0)} W \cup \partial^{(1)} W$, where $\partial^{(1)} W$ fibers over a manifold with boundary $\beta W$,

$$
\partial^{(0)} W=M \sqcup-M^{\prime}, \quad \partial\left(\partial^{(1)} W\right)=\partial M \sqcup-\partial M^{\prime}, \quad \partial(\beta W)=\beta M \sqcup-\beta M^{\prime},
$$

i.e., $\partial^{(1)} W: \partial M \rightsquigarrow \partial M^{\prime}$ and $\beta W: \beta M \rightsquigarrow \beta M^{\prime}$ are usual spin bordisms between closed spin manifolds, and

$$
\partial^{(0)} W \cap \partial^{(1)} W=\partial M \sqcup-\partial M^{\prime} .
$$

We also have a principal $G$-bundle $\bar{P} \stackrel{\bar{p}}{\rightarrow} \beta W$ restricting to $P$ and $P^{\prime}$ over $\partial(\beta W)=\beta M \sqcup-\beta M^{\prime}$. A psc-geometric cycle for $\operatorname{Pos}_{n}^{\text {spin, }(L, G) \text {-fb }}\left(B \Gamma_{\beta} \stackrel{B \theta}{\longrightarrow} B \Gamma\right)$ is then given by a tuple

$$
\left(M, P \stackrel{p}{\rightarrow} \beta M, \xi, \xi_{\beta}, g_{M}, g_{\beta M}, \nabla^{p}\right),
$$

where $g_{M}$ and $g_{\beta M}$ are Riemannian metrics of psc and $\nabla^{p}$ is a bundle-connection on $P$. Then two psc-geometric cycles

$$
\left(M, P \stackrel{p}{\rightarrow} \beta M, \xi, \xi_{\beta}, g_{M}, g_{\beta M}, \nabla^{p}\right) \quad \text { and } \quad\left(M^{\prime}, P^{\prime} \stackrel{p^{\prime}}{\rightarrow} \beta M^{\prime}, \xi^{\prime}, \xi_{\beta}^{\prime}, g_{M^{\prime}}, g_{\beta M^{\prime}}, \nabla^{P^{\prime}}\right)
$$

are equivalent if there exists a bordism $(W, \bar{P})$ as above together with metrics of psc $\bar{g}_{W}$ and $\bar{g}_{\beta W}$ restricting to $g_{M}, g_{M^{\prime}}$ and $g_{\beta M}, g_{\beta M^{\prime}}$ on the boundary, along with a connection $\nabla^{\bar{P}}$ restricting to the connections $\nabla^{p}$ and $\nabla^{P^{\prime}}$. The proof of the following statement is straightforward and thus omitted.

Proposition 6.11. There exist natural homomorphisms

$$
\begin{aligned}
& R^{\mathrm{Pos}}: \operatorname{Pos}_{n}^{\mathrm{spin},(L, G)-\mathrm{fb}}\left(B \Gamma_{\beta} \stackrel{B \theta}{\longrightarrow} B \Gamma\right) \rightarrow \operatorname{Pos}_{n}^{\mathrm{spin},(L, G)-\mathrm{fb}}(B \Gamma) \\
& \text { "forget } \Gamma_{\beta} \text { ", } \\
& i^{\text {Pos }}: \operatorname{Pos}_{n}^{\text {spin }}(B \Gamma) \rightarrow \operatorname{Pos}_{n}^{\text {spin },(L, G)-\mathrm{fb}}\left(B \Gamma_{\beta} \stackrel{B \theta}{\longrightarrow} B \Gamma\right) \\
& \text { "consider a closed manifold as a pseudomanifold with empty singularities", } \\
& f_{\Gamma_{\beta}, \Gamma}: \operatorname{Pos}_{n}^{\text {spin },(L, G)-\mathrm{fb}}\left(B \Gamma_{\beta} \stackrel{B \theta}{\longrightarrow} B \Gamma\right) \rightarrow \Omega_{n}^{\mathrm{spin},(L, G)-\mathrm{fb}}\left(B \Gamma_{\beta} \stackrel{B \theta}{\longrightarrow} B \Gamma\right) \\
& \text { "forget metric and connection". }
\end{aligned}
$$

We also have a natural map

$$
\pi_{0}\left(\mathcal{R}_{w}^{+}\left(M_{\Sigma}\right)\right) \rightarrow \operatorname{Pos}_{n}^{\mathrm{spin},(L, G)-\mathrm{fb}}\left(B \Gamma_{\beta} \stackrel{B \theta}{\longrightarrow} B \Gamma\right) .
$$

We would like to use the technique of Botvinnik-Gilkey [12] (and generalizations of it, such as the ones presented in [37]) in order to detect elements in the groups

$$
\operatorname{Pos}_{n}^{\text {spin },(L, G)-\mathrm{fb}}\left(B \pi_{1}(\beta M) \rightarrow B \pi_{1}\left(M_{\Sigma}\right)\right),
$$

with $M_{\Sigma}$ a pseudomanifold with $(L, G)$-fibered singularities. A relevant version of the rho invariant on a depth-one wedge spin stratified pseudomanifold of psc was introduced in [39]. Then, using a suitable APS index theorem on spaces with $(L, G)$-fibered singularities, it follows from [39] that the APS rho invariant and the Cheeger-Gromov rho invariant of such a $M_{\Sigma}$ are well defined and that they both define maps $\rho_{w, \mathrm{APS}}: \pi_{0}\left(\mathcal{R}_{w}^{+}\left(M_{\Sigma}\right)\right) \rightarrow \mathbb{R}$ and $\rho_{w, \mathrm{CG}}: \pi_{0}\left(\mathcal{R}_{w}^{+}\left(M_{\Sigma}\right)\right)$ $\rightarrow \mathbb{R}$ and group homomorphisms:

$$
\rho_{w, \mathrm{APS}}: \operatorname{Pos}_{n}^{\mathrm{spin},(L, G)-\mathrm{fb}}(B \Gamma) \rightarrow \mathbb{R}, \quad \rho_{w, \mathrm{CG}}: \operatorname{Pos}_{n}^{\mathrm{spin},(L, G)-\mathrm{fb}}(B \Gamma) \rightarrow \mathbb{R},
$$


with $\Gamma=\pi_{1}\left(M_{\Sigma}\right)$. We shall mainly be interested in $\rho_{w, \mathrm{CG}}$ and we shall denote it simply by $\rho_{w}$. Summarizing, we have a well-defined homomorphism:

$$
\rho_{w}: \operatorname{Pos}_{n}^{\text {spin },(L, G)-\mathrm{fb}}(B \Gamma) \rightarrow \mathbb{R} .
$$

Here is an example where the Cheeger-Gromov rho invariant of a wedge space can be used in order to show that the group $\operatorname{Pos}_{n}^{\text {spin, }(L, G)-\mathrm{fb}}\left(B \pi_{1}(\beta M) \rightarrow B \pi_{1}\left(M_{\Sigma}\right)\right)$ is infinite.

Proposition 6.12. Let $L$ be simply connected, $G$ be a compact semisimple Lie group. Let $M_{\Sigma}$ be a pseudomanifold with $(L, G)$-fibered singularities and of dimension $m=4 k+3$, with $\Gamma:=$ $\pi_{1}\left(M_{\Sigma}\right)$. Assume that $\Gamma$ has an element of finite order. Then the group

$$
\operatorname{Pos}_{m}^{\text {spin, }(L, G)-\mathrm{fb}}\left(B \pi_{1}(\beta M) \rightarrow B \pi_{1}\left(M_{\Sigma}\right)\right)
$$

is infinite provided $\mathcal{R}_{w}^{+}\left(M_{\Sigma}\right) \neq \varnothing$.

Proof. Let $\Gamma_{\beta}:=\pi_{1}(\beta M), \Gamma:=\pi_{1}\left(M_{\Sigma}\right)$ and $\theta: \Gamma_{\beta} \rightarrow \Gamma$ induced by the inclusion of $\beta M$ into $M_{\Sigma}$. Let $\xi_{\Sigma}: M_{\Sigma} \rightarrow B \Gamma$ be the classifying map for the universal cover of $M_{\Sigma}$; this provides us with a map $\xi: M \rightarrow B \Gamma$ and we also have a classifying map $\xi_{\beta}: \beta M \rightarrow B \Gamma_{\beta}$ satisfying the compatibility conditions of Definition 4.5. As already remarked, we have a forgetful homomorphism

$$
f_{\Gamma_{\beta}, \Gamma}: \operatorname{Pos}_{m}^{\mathrm{spin},(L, G)-\mathrm{fb}}\left(B \Gamma_{\beta} \stackrel{B \theta}{\longrightarrow} B \Gamma\right) \rightarrow \Omega_{m}^{\mathrm{spin},(L, G)-\mathrm{fb}}\left(B \Gamma_{\beta} \stackrel{B \theta}{\longrightarrow} B \Gamma\right),
$$

(we forget the metrics). We shall consider the subset $C\left(M_{\Sigma}, \xi, \xi_{\beta}\right)$ of the group $\operatorname{Pos}_{m}^{\mathrm{spin},(L, G)-\mathrm{fb}}\left(B \Gamma_{\beta}\right.$ $\stackrel{B \theta}{\longrightarrow} B \Gamma$ ) obtained by keeping $M_{\Sigma}$ and the classifying maps $\xi: M \rightarrow B \Gamma$ and $\xi_{\beta}: \beta M \rightarrow B \Gamma_{\beta}$ fixed and varying only the psc metrics $g_{M}, g_{\beta M}$ and the connection $\nabla^{p}$.

We shall prove that under the present assumptions

- $\operatorname{ker} f_{\Gamma_{\beta}, \Gamma}$ has infinite cardinality,

- there is a free and transitive action of $\operatorname{ker} f_{\Gamma_{\beta}, \Gamma}$ on $C\left(M_{\Sigma}, \xi, \xi_{\beta}\right)$.

This will imply that $C\left(M_{\Sigma}, \xi, \xi_{\beta}\right)$ and thus $\operatorname{Pos}_{m}^{\text {spin, }(L, G) \text {-fb }}\left(B \Gamma_{\beta} \stackrel{B \theta}{\longrightarrow} B \Gamma\right)$ has infinite cardinality. Consider then $\operatorname{ker}\left(f_{\Gamma_{\beta}, \Gamma}\right) \subset \operatorname{Pos}_{m}^{\text {spin, }(L, G) \text {-fb }}\left(B \Gamma_{\beta} \stackrel{B \theta}{\longrightarrow} B \Gamma\right)$ and $C\left(M_{\Sigma}, \xi, \xi_{\beta}\right)$. Then, using crucially our bordism theorem, Theorem 4.11, we can prove as in [37, Proposition 2.4], that there is well defined action

$$
\operatorname{ker}\left(f_{\Gamma_{\beta}, \Gamma}\right) \times C\left(M_{\Sigma}, \xi, \xi_{\beta}\right) \rightarrow C\left(M_{\Sigma}, \xi, \xi_{\beta}\right)
$$

which associates to $x \in \operatorname{ker}\left(f_{\Gamma_{\beta}, \Gamma}\right)$ and $\left[\left(M_{\Sigma}, \xi, \xi_{\beta}, g_{M}, g_{\beta M}\right)\right]^{8}$ the class

$$
x+\left[\left(M_{\Sigma}, \xi, \xi_{\beta}, g_{M}, g_{\beta M}\right)\right] .
$$

Indeed, as $x$ is null-bordant in $\Omega_{m}^{\mathrm{spin},(L, G)-\mathrm{fb}}\left(B \Gamma_{\beta} \stackrel{B \theta}{\longrightarrow} B \Gamma\right)$, the element $x+\left[\left(M_{\Sigma}, \xi, \xi_{\beta}\right)\right]$ is bordant to $\left[\left(M_{\Sigma}, \xi, \xi_{\beta}\right)\right]$ in $\Omega_{m}^{\text {spin, }(L, G)-\mathrm{fb}}\left(B \Gamma_{\beta} \stackrel{B \theta}{\longrightarrow} B \Gamma\right)$. Since the element $x+\left[\left(M_{\Sigma}, \xi, \xi_{\beta}\right)\right]$ is represented by a manifold with an adapted wedge psc metric, we can use our bordism theorem and propagate this psc metric back to $M_{\Sigma}$, obtaining a new adapted wedge metric of psc, i.e., a new element in $C\left(M_{\Sigma}, \xi_{\beta}, \xi\right)$. One proves exactly as in Proposition 2.4 in [37] that this action is well defined, free and transitive.

\footnotetext{
${ }^{8}$ Here we skip " $\nabla^{p} "$ from the notations.
} 
Consider now $\Gamma=\mathbb{Z}_{n}$, the cyclic group of order $n$. It is explained in [37], building on specific examples provided in [12], that the Cheeger-Gromov rho invariant defines a map $\rho$ : $\operatorname{Pos}_{m}^{\text {spin }}\left(B \mathbb{Z}_{n}\right) \rightarrow \mathbb{R}$ which has an image of infinite cardinality. To prove this, one only needs to show that $\rho$ is a homomorphism and that it is non-trivial; the property that it is a homomorphism is a consequence of the definition of rho invariant whereas the fact that it is non-trivial follows from the specific examples in [12] (lens spaces). Remark that we have a well defined rho-homomorphism $\rho_{w}^{\text {rel }}: \operatorname{Pos}_{m}^{\text {spin, }(L, G) \text {-fb }}\left(B \Gamma_{\beta} \stackrel{B \theta}{\longrightarrow} B \Gamma\right) \rightarrow \mathbb{R}$, obtained by composing the homomorphism (6.7) with the homomorphism (6.10):

$$
\rho_{w}^{\mathrm{rel}}:=\rho_{w} \circ R^{\mathrm{Pos}} \text {. }
$$

Recall now that we are under the assumption that there is an injection $j: \mathbb{Z}_{n} \rightarrow \Gamma$. Such an injection induces homomorphisms

$$
B j_{*}^{\Omega}: \Omega_{m}^{\text {spin }}\left(B \mathbb{Z}_{n}\right) \rightarrow \Omega_{m}^{\text {spin }}(B \Gamma) \quad \text { and } \quad B j_{*}^{\text {Pos }}: \operatorname{Pos}_{m}^{\text {spin }}\left(B \mathbb{Z}_{n}\right) \rightarrow \operatorname{Pos}_{m}^{\text {spin }}(B \Gamma) .
$$

Consider the following diagram, where for typographic reasons we omit the superscripts spin and $\mathrm{fb}$,

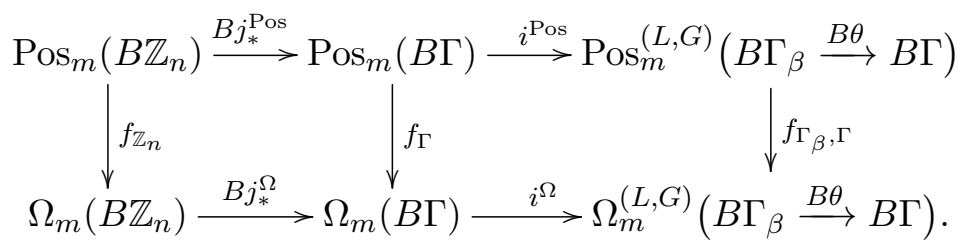

By naturality this is a commutative diagram. Consider $K:=\operatorname{ker} f_{\mathbb{Z}_{n}}$. We know from [37] that $\left.\rho\right|_{K}$ has an image of infinite cardinality. For $\kappa \in K$ consider

$$
\Theta_{\kappa}:=i^{\mathrm{Pos}} \circ B j_{*}^{\mathrm{Pos}}(\kappa) \in \operatorname{Pos}_{m}^{\mathrm{spin},(L, G)-\mathrm{fb}}\left(B \Gamma_{\beta} \stackrel{B \theta}{\longrightarrow} B \Gamma\right) .
$$

Then, by commutativity of the diagram, we have

$$
f_{\Gamma_{\beta}, \Gamma}\left(\Theta_{\kappa}\right)=0 .
$$

This means that

$$
\left\{\Theta_{k}+\left[M, \beta M, \xi, \xi_{\beta}, g\right], \kappa \in K\right\} \subset C\left(M_{\Sigma}, \xi, \xi_{\beta}\right) \subset \operatorname{Pos}_{m}^{\mathrm{spin},(L, G)-\mathrm{fb}}\left(B \Gamma_{\beta} \stackrel{B \theta}{\longrightarrow} B \Gamma\right) .
$$

We also have

$$
\rho_{w}^{\mathrm{rel}}\left(\Theta_{k}+\left[M, \beta M, \xi, \xi_{\beta}, g_{M}, g_{\beta M}, \nabla^{p}\right]\right)=\rho(k)+\rho_{w}\left[M_{\Sigma}, \xi_{\Sigma}, g\right]
$$

with $g$ the wedge metric defined by $g_{M}, g_{\beta M}$ and $\nabla^{p}$. Indeed:

$$
\begin{aligned}
\rho_{w}^{\mathrm{rel}}\left(\left[M, \beta M, \xi, \xi_{\beta}, g_{M}, g_{\beta M}, \nabla^{p}\right]\right): & =\rho_{w}\left(R^{\mathrm{Pos}}\left(\left[M, \beta M, \xi, \xi_{\beta}, g_{M}, g_{\beta M}, \nabla^{p}\right]\right)\right) \\
& =\rho_{w}\left[M_{\Sigma}, \xi_{\Sigma}, g\right],
\end{aligned}
$$

whereas, thanks to Lemma 2.2 in [37] and naturality, we have

$$
\rho_{w}^{\mathrm{rel}}\left(\Theta_{k}\right)=\rho_{w}^{\mathrm{rel}}\left(i^{\mathrm{Pos}} \circ B j_{*}^{\mathrm{Pos}}(\kappa)\right)=\rho_{w}\left(R^{\mathrm{Pos}} \circ i^{\mathrm{Pos}} \circ B j_{*}^{\mathrm{Pos}}(\kappa)\right)=\rho\left(B j_{*}^{\mathrm{Pos}}(\kappa)\right)=\rho(\kappa) .
$$

This implies that the set $\left\{\Theta_{k}+\left[M, \beta M, \xi, \xi_{\beta}, g\right], \kappa \in K\right\}$ and thus the group

$$
\operatorname{Pos}_{m}^{\mathrm{spin},(L, G)-\mathrm{fb}}\left(B \Gamma_{\beta} \stackrel{B \theta}{\longrightarrow} B \Gamma\right)
$$

that contains it, is of infinite cardinality. 
Corollary 6.13. Under the previous assumptions we have

$$
\left|\pi_{0}\left(\mathcal{R}_{w}^{+}\left(M_{\Sigma}\right)\right)\right|=\infty
$$

Proof. It suffices to observe that there exists a surjection $\pi_{0}\left(\mathcal{R}_{w}^{+}\left(M_{\Sigma}\right)\right) \rightarrow C\left(M_{\Sigma}, \xi, \xi_{\beta}\right)$.

The above result is just an example of how rho-invariants can be used in order to detect elements in bordism groups of wedge psc metrics or in $\pi_{0}\left(\mathcal{R}_{w}^{+}\left(M_{\Sigma}\right)\right)$. It should also be possible to use higher rho invariants to get sharper results (under additional assumptions on the fundamental groups). We comment on this in the next section.

\section{Open problems and subjects for future study}

In this section we just sketch a few ideas for additional projects related to the topics of this paper.

\subsection{The index-difference homomorphism for $L$-fibered pseudomanifolds}

In this paper, we have not done very much (beyond the obstruction theory in Section 2) for $L$ fibered pseudomanifolds that are not $(L, G)$-fibered. But there is hope of extending the theory of the index-difference homomorphism to the more general $L$-fibered case. Assume that $g_{0}, g_{1} \in$ $\mathcal{R}_{w}^{+}\left(M_{\Sigma}\right)$, with $M_{\Sigma}$ an $L$-fibered pseudomanifold. Let us take a path of wedge metrics joining $g_{0}$ and $g_{1}$ and let us denote by $g_{0}^{v}$ and $g_{1}^{v}$ the vertical part of the metric on the link-bundle. It is not automatic, in this generality, that the path we take joining $g_{0}$ and $g_{1}$ will stay within the metrics that are geometric-Witt or psc-Witt, i.e., with positive scalar curvature along the links. Under which assumptions can we ensure this? One sufficient condition is that $\mathcal{R}^{+}(\partial M / \beta M)$, the space of vertical metrics of psc along the links, is connected, and, moreover, that we can lift a path joining $g_{0}^{v}$ and $g_{1}^{v}$ in $\mathcal{R}^{+}(\partial M / \beta M)$ to a path joining $g_{0}$ and $g_{1}$ in $\mathcal{R}_{w}^{+}\left(M_{\Sigma}\right)$. When this is the case, the theory of the index difference outlined above in Section 6.2 ought to go through; of course, it will be necessary to work out the details.

\subsection{The theory of $R$-groups}

In [44], Stolz sketched the theory of concordance groups of psc metrics (on compact smooth manifolds), which he called $R$-groups. Assuming for simplicity that we are considering spin manifolds with fundamental group $\Gamma$, one obtains a long exact sequence [44, sequence (4.4)]

$$
\cdots \rightarrow R_{n+1}^{\text {spin }}(B \Gamma) \stackrel{\partial}{\rightarrow} \operatorname{Pos}_{n}^{\text {spin }}(B \Gamma) \rightarrow \Omega_{n}^{\text {spin }}(B \Gamma) \rightarrow R_{n}^{\text {spin }}(B \Gamma) \stackrel{\partial}{\rightarrow} \cdots
$$

Here $\operatorname{Pos}_{n}^{\text {spin }}(B \Gamma)$ is the bordism group of pairs $(M, g)$, with $M$ a closed spin manifold with a map to $B \Gamma$ and $g$ a positive scalar curvature metric on $M$. The map from this group to $\Omega_{n}^{\text {spin }}(B \Gamma)$ simply forgets the Riemannian metric. The group $R_{n}^{\text {spin }}(B \Gamma)$ [44, Definition 4.1] is a bordism group of pairs $(M \rightarrow B \Gamma, h)$, with $M$ a compact spin $n$-manifold with possibly non-empty boundary, and with $h$ a psc metric on $\partial M$. The map $\partial: R_{n+1}^{\mathrm{spin}}(B \Gamma) \rightarrow \operatorname{Pos}_{n}^{\mathrm{spin}}(B \Gamma)$ is just restriction to the boundary.

It should be rather straightforward to replace $\operatorname{Pos}_{n}^{\text {spin }}(B \Gamma)$ here with $\operatorname{Pos}_{n}^{\text {spin, }(L, G)-\mathrm{fb}}(B \Gamma)$, and $\Omega_{n}^{\text {spin }}(B \Gamma)$ with $\Omega_{n}^{\text {spin, }(L, G) \text {-fb }}(B \Gamma)$, to obtain $R$-groups of pseudomanifolds with $(L, G)$-fibered singularities: $R_{n}^{\mathrm{spin},(L, G)-\mathrm{fb}}(B \Gamma)$.

- What do these $R$-groups look like and how do they differ from the usual $R$-groups?

- Do they act freely and transitively on the set of concordance classes of psc wedge metrics, as in Stolz [44]? 
- Do we have the analogue of Stolz sequence (7.1) in the singular setting?

- If so, can one map the analogue of sequence (7.1) to a Higson-Roe type analytic exact sequence as in [38]? This would involve the definition of a higher rho class associated to a wedge psc metric. For the signature operator on stratified spaces that are Witt or Cheeger, a similar result has been proved in [5], mapping the Browder-Quinn surgery sequence to the Higson-Roe analytic surgery sequence, and we expect the analysis there to play a role here.

- Is there an $R$-group $R_{n}^{\mathrm{spin},(L, G)-\mathrm{fb}}\left(B \Gamma_{\beta} \stackrel{B \theta}{\longrightarrow} B \Gamma\right)$ and does it fit into a Stolz sequence

$$
\begin{aligned}
\cdots & \rightarrow \Omega_{n}^{\mathrm{spin},(L, G)-\mathrm{fb}}\left(B \Gamma_{\beta} \stackrel{B \theta}{\longrightarrow} B \Gamma\right) \rightarrow R_{n}^{\mathrm{spin},(L, G)-\mathrm{fb}}\left(B \Gamma_{\beta} \stackrel{B \theta}{\longrightarrow} B \Gamma\right) \\
& \rightarrow \operatorname{Pos}_{n-1}^{\mathrm{spin},(L, G)-\mathrm{fb}}\left(B \Gamma_{\beta} \stackrel{B \theta}{\longrightarrow} B \Gamma\right) \rightarrow \cdots ?
\end{aligned}
$$

- Can we use the Stolz $(L, G)$-sequences above, the mapping to Higson-Roe, and the techniques of $\mathrm{Xie}-\mathrm{Yu}$-Zeidler [49] in order to give a lower bound on the rank of the group $\operatorname{Pos}_{n}^{\operatorname{spin},(L, G)-\mathrm{fb}}(B \Gamma)$ ?

\subsection{Rho-invariants of wedge psc metrics}

\subsubsection{Torsion-free groups}

In the smooth case one can prove that if $\pi_{1}(M)$ is torsion-free and the Baum-Connes map $\mu_{\max }: K_{*}(B \Gamma) \rightarrow K_{*}\left(C_{\max }^{*} \Gamma\right)$ is an isomorphism, then the Cheeger-Gromov rho invariant of a positive scalar curvature metric on a spin manifold $M$ of odd dimension must vanish. See [36] and also [8] for a new proof based on the Higson-Roe analytic surgery sequence. Notice that this is a no-go result: the Cheeger-Gromov rho invariant for manifolds with fundamental group satisfying these conditions cannot be used in order to distinguish metrics of psc (indeed, it is equal to 0$)$.

Can one extend this vanishing result to $(L, G)$-pseudomanifolds and the Cheeger-Gromov rho invariant of a wedge metric?

A direct approach would build on the proof of Piazza-Schick in [36]. A different route would use the results of the previous Section 6.4, the Benameur-Roy map $K_{0}\left(D_{\Gamma}^{*}\right) \rightarrow \mathbb{R}$ of [8] and the exactness of the Higson-Roe analytic surgery sequence.

\subsubsection{Groups with torsion}

On the other hand, one would like to define and use higher rho invariants in order to distinguish wedge metrics of positive scalar curvature, especially for fundamental groups with torsion. See for example $[6,33,48]$ for the case of smooth closed manifolds.

\subsection{Stratifications with higher depth}

For applications to algebraic varieties, moduli spaces, and other natural examples of stratified spaces, it would be nice to generalize our theory to pseudomanifolds with higher depth, in other words, with singular strata of multiple dimensions. While the analytic part concerning Dirac operators is largely under control, thanks to [1] (see also [40]), on the geometric side we expect several complications:

- The geometry of the tubular neighborhoods of the singular strata gets to be rather complicated, as one would need to consider iterated Riemannian submersions and careful curvature estimates. 
- Proving the appropriate bordism theorem would be quite complicated.

- Perhaps one could say more in the case of Baas-Sullivan singularities, as in [10], which did consider some pseudomanifolds with higher depth.

\subsection{Topological questions}

- The "mixed fundamental group" bordism group $\Omega_{n}^{(L, G)}\left(B \Gamma_{\beta} \stackrel{B \theta}{\longrightarrow} B \Gamma\right)$ seems to be very hard to compute; it doesn't just fit into an exact sequence like the one we developed in [14]. But is there a spectral sequence computing $\Omega_{n}^{(L, G)}\left(B \Gamma_{\beta} \stackrel{B \theta}{\longrightarrow} B \Gamma\right)$ ?

- What about a Kreck-Stolz $s$-invariant? More precisely, in [30, Proposition 2.13], Kreck and Stolz defined an invariant $s(M, g)$ of psc metrics $g$ on closed spin manifolds $M$ of dimension $3 \bmod 4$ with vanishing Pontryagin classes. This invariant has the properties that it is preserved under spin-structure-preserving isometries and that the relative index $i\left(g, g^{\prime}\right)$ in the sense of [26, Section 4] is given by $s(M, g)-s\left(M, g^{\prime}\right)$. It would be interesting to know if one could do something similar for adapted psc wedge metrics on Witt spin pseudomanifolds $M_{\Sigma}$. This would require using the index theorem of [39] and replacing vanishing of the Pontryagin classes with vanishing of the Goresky-MacPherson homology $L$-class or, more generally, of the $K$-homology class of the pseudomanifold signature operator.

\section{A On the spin-bordism invariance of the $\alpha$-invariant}

In this appendix we shall discuss briefly an analytic proof of the spin-bordism invariance of the $\alpha$ class and of the $\alpha^{\Gamma}$ class. We have already observed, building on the case of the signature operator on Witt spaces treated in great detail in [4, Section 7], that given an analytic proof in the smooth case, it extends mutatis mutandis to the pseudomanifold case.

\section{A.1 A generator of $K O_{1}(\mathbb{R})$}

Consider $\mathbb{R}$ with its standard spin structure. Then we have $\mathfrak{P}_{\mathbb{R}}$ and its class in $K O_{1}(\mathbb{R}):=$ $K K O\left(C_{0}(\mathbb{R}), C \ell_{1}\right)$.

Proposition A.1. $K O_{1}(\mathbb{R})$ is isomorphic to $\mathbb{Z}$ and the class $\left[\mathscr{D}_{\mathbb{R}}\right] \in K O_{1}(\mathbb{R})$ is a generator. Equivalently, $K O_{1}((0,1))$ is isomorphic to $\mathbb{Z}$ and the class $\left[\mathfrak{P}_{(0,1)}\right] \in K O_{1}((0,1))$ is a generator.

Proof. This is a very special case of [28, Theorems 4.8 and 4.10] and of [29, Section 5, Lemma 4].

\section{A.2 Boundary of Atiyah-Singer is Atiyah-Singer}

We now know that $\left[\mathfrak{P}_{\mathbb{R}}\right]$ is the generator of $K O_{1}(\mathbb{R})$. We want to prove that prove that "boundary of Atiyah-Singer is Atiyah-Singer" in $K O$-homology, i.e.,

$$
\delta\left[\mathfrak{P}_{X}\right]=\left[\mathfrak{P}_{\partial X}\right] \quad \text { in } K O_{\operatorname{dim} \partial X}(\partial X)
$$

on a spin manifold $X$ with metrically collared boundary $\partial X$. Here $\delta$ is the connecting homomorphism in KO-theory associated to the semisplit short exact sequence

$$
0 \rightarrow C_{\partial X}(X) \rightarrow C(X) \rightarrow C(\partial X) \rightarrow 0
$$

with $C_{\partial X}(X)$ the ideal of continuous functions vanishing on the boundary.

Using excision we can easily reduce to a collar neighbourhood. Then the result is a consequence of the following: 
Lemma A.2. Let $X=[0,1) \times N$ with $\partial X=\{0\} \times N$. Let $n=\operatorname{dim} N$. In this case $C_{\partial X}(X)=$ $C_{0}((0,1) \times N)$. Let us consider the connecting homomorphism

$$
\left.K O_{n+1}((0,1) \times N)\right) \stackrel{\delta}{\rightarrow} K O_{n}(N)
$$

associated to the semisplit short exact sequence

$$
0 \rightarrow C_{0}((0,1) \times N) \rightarrow C_{0}([0,1) \times N) \rightarrow C(N) \rightarrow 0 .
$$

Then $\delta$ is the inverse of the isomorphism

$$
K O_{n}(N) \rightarrow K O_{n+1}((0,1) \times N)
$$

obtained by taking the Kasparov product with $\left[\mathfrak{P}_{(0,1)}\right]$, the generator of $K O_{1}((0,1))$. In particular, as $\left[\mathfrak{P}_{(0,1) \times N}\right]$ is precisely the Kasparov product of $\left[\mathfrak{P}_{(0,1)}\right]$ and $\left[\mathfrak{P}_{N}\right]$, we have that $\delta\left[\mathfrak{P}_{(0,1) \times N}\right]=\left[\mathfrak{P}_{N}\right]$ which is what we wanted to prove.

Proof. By the naturality of Kasparov product we can reduce to the connecting homomorphism

$$
K O_{1}((0,1)) \stackrel{\delta}{\rightarrow} K O_{0} \text { (point) }
$$

associated to the semisplit short exact sequence

$$
0 \rightarrow C_{0}((0,1)) \rightarrow C_{0}([0,1)) \rightarrow \mathbb{R} \rightarrow 0 .
$$

But $\delta$ is the inverse of the isomorphism $K O_{0}$ (point) $\rightarrow K O_{1}((0,1))$ obtained by taking the Kasparov product with the generator $\left[\mathfrak{P}_{(0,1)}\right]$ of $K O_{1}((0,1))$. This again follows from Kasparov's results on Poincaré duality in KO-theory, [28, Theorems 4.8 and 4.10] and [29, Section 5, Lemma 4].

\section{A.3 Spin bordism invariance}

Using $\delta\left[\mathscr{P}_{X}\right]=\left[\mathfrak{D}_{\partial X}\right]$ we can now prove the spin bordism invariance of the $\alpha$-class in $K O_{n}$.

Theorem A.3. Assume that $Y$ is spin and of dimension $n$ and that $Y=\partial X$, with $X$ spin. Then $\alpha(Y)=0 \in K O_{n}$.

Proof. We write part of the long exact sequence in $K O$ associated to the short exact sequence

$$
0 \rightarrow C_{\partial X}(X) \rightarrow C(X) \rightarrow C(\partial X) \rightarrow 0 .
$$

We have the induced exact sequence

$$
K O_{n+1}(X, \partial X) \stackrel{\delta}{\rightarrow} K O_{n}(\partial X) \stackrel{\iota_{*}}{\rightarrow} K O_{n}(X)
$$

with $\iota$ the inclusion of $\partial X$ into $X$. Notice, in particular, that

$$
\iota_{*} \circ \delta=0 .
$$

Consider $\pi^{Y}: Y \rightarrow$ point; obviously $\pi^{Y}=\pi^{X} \circ \iota$. Then we have

$$
\alpha(Y)=\pi_{*}^{Y}\left[\mathfrak{P}_{Y}\right]=\pi_{*}^{X} \circ \iota_{*}\left[\mathfrak{D}_{Y}\right]=\pi_{*}^{X} \circ \iota_{*} \circ \delta\left[\mathfrak{D}_{X}\right]=0 .
$$

The same proof applies to the $\alpha^{\Gamma}$ class if we use the Atiyah-Singer-Mishchenko operator $\mathscr{P}_{\mathrm{MF}}$ and the exact sequence

$$
K K_{n+1} O\left(C_{\partial X}(X), C_{r}^{*} \Gamma\right) \stackrel{\delta}{\rightarrow} K K_{n} O\left(C(\partial X), C_{r}^{*} \Gamma\right) \stackrel{\iota *}{\rightarrow} K K_{n} O\left(C_{\partial X}(X), C_{r}^{*} \Gamma\right) .
$$

See [32, Proposition 2.3] for the details.

Note that for the $\alpha$ class the spin bordism invariance is usually proved by identifying it with the Atiyah-Milnor-Singer invariant. See [31]. The generalization of this approach to the $\alpha^{\Gamma}$ class is treated in [41] and (more extensively) in [42]. 


\section{Acknowledgements}

We thank the Mathematisches Forschungsinstitut Oberwolfach for hosting Workshop 1732 in 2017 on Analysis, Geometry and Topology of Positive Scalar Curvature Metrics, which marked the start of this project. This work was also supported by U.S. NSF grant number DMS-1607162, by Simons Foundation Collaboration Grant number 708183, by Sapienza Università di Roma, and by the Ministero Istruzione Università e Ricerca through the PRIN Spazi di Moduli e Teoria di Lie. B.B. and J.R. acknowledge a very pleasant and productive visit to Rome in May-June 2019, as well as a visit by J.R. to Rome in January 2020. P.P. thanks Pierre Albin and Jesse Gell-Redman for interesting discussions about the content of Section 2.

We would like to thank the referees of this article for a careful reading of the previous drafts and for suggestions for improvements.

\section{References}

[1] Albin P., Gell-Redman J., The index formula for families of Dirac type operators on pseudomanifolds, arXiv:1712.08513.

[2] Albin P., Gell-Redman J., The index of Dirac operators on incomplete edge spaces, SIGMA 12 (2016), 089, 45 pages, arXiv:1312.4241.

[3] Albin P., Gell-Redman J., Piazza P., Higher index theory for Dirac operators on wedge pseudomanifolds, in preparation.

[4] Albin P., Leichtnam E., Mazzeo R., Piazza P., The signature package on Witt spaces, Ann. Sci. Éc. Norm. Supér. (4) 45 (2012), 241-310, arXiv:1112.0989.

[5] Albin P., Piazza P., Stratified surgery and K-theory invariants of the signature operator, Ann. Sci. Éc. Norm. Supér. (4), to appear, arXiv:1710.00934.

[6] Azzali S., Wahl C., Two-cocycle twists and Atiyah-Patodi-Singer index theory, Math. Proc. Cambridge Philos. Soc. 167 (2019), 437-487, arXiv:1312.6373.

[7] Baum P., Douglas R.G., Taylor M.E., Cycles and relative cycles in analytic $K$-homology, J. Differential Geom. 30 (1989), 761-804.

[8] Benameur M.-T., Roy I., The Higson-Roe exact sequence and $\ell^{2}$ eta invariants, J. Funct. Anal. 268 (2015), 974-1031, arXiv:1409.2717.

[9] Bismut J.-M., Cheeger J., $\eta$-invariants and their adiabatic limits, J. Amer. Math. Soc. 2 (1989), 33-70.

[10] Botvinnik B., Manifolds with singularities accepting a metric of positive scalar curvature, Geom. Topol. 5 (2001), 683-718, arXiv:math.DG/9910177.

[11] Botvinnik B., Ebert J., Randal-Williams O., Infinite loop spaces and positive scalar curvature, Invent. Math. 209 (2017), 749-835, arXiv:1411.7408.

[12] Botvinnik B., Gilkey P., The eta invariant and metrics of positive scalar curvature, Math. Ann. 302 (1995), $507-517$.

[13] Botvinnik B., Gilkey P., Stolz S., The Gromov-Lawson-Rosenberg conjecture for groups with periodic cohomology, J. Differential Geom. 46 (1997), 374-405.

[14] Botvinnik B., Piazza P., Rosenberg J., Positive scalar curvature on simply connected spin pseudomanifolds, J. Topol. Anal., to appear, arXiv:1908.04420.

[15] Botvinnik B., Rosenberg J., Positive scalar curvature on manifolds with fibered singularities, arXiv:1808.06007.

[16] Botvinnik B., Walsh M.G., Homotopy invariance of the space of metrics with positive scalar curvature on manifolds with singularities, SIGMA 17 (2021), 034, 27 pages, arXiv:2005.03073.

[17] Buggisch L., The spectral flow theorems for families of twisted Dirac operators, Ph.D. Thesis, University of Münster, 2018, available at https://d-nb.info/1190724960/34.

[18] Bunke U., A $K$-theoretic relative index theorem and Callias-type Dirac operators, Math. Ann. 303 (1995), 241-279.

[19] Debord C., Lescure J.-M., Rochon F., Pseudodifferential operators on manifolds with fibred corners, Ann. Inst. Fourier (Grenoble) 65 (2015), 1799-1880, arXiv:1112.4575.

[20] Dwyer W., Schick T., Stolz S., Remarks on a conjecture of Gromov and Lawson, in High-Dimensional Manifold Topology, World Sci. Publ., River Edge, NJ, 2003, 159-176, arXiv:math.GT/0208011.

[21] Ebert J., The two definitions of the index difference, Trans. Amer. Math. Soc. 369 (2017), 7469-7507, arXiv:1308.4998. 
[22] Ebert J., Index theory in spaces of manifolds, Math. Ann. 374 (2019), 931-962, arXiv:1608.01701.

[23] Ebert J., Frenck G., The Gromov-Lawson-Chernysh surgery theorem, Bol. Soc. Mat. Mex. 27 (2021), 37, 43 pages, arXiv:1807.06311.

[24] Ebert J., Randal-Williams O., Infinite loop spaces and positive scalar curvature in the presence of a fundamental group, Geom. Topol. 23 (2019), 1549-1610, arXiv:1711.11363.

[25] Gromov M., Lawson Jr. H.B., The classification of simply connected manifolds of positive scalar curvature, Ann. of Math. 111 (1980), 423-434.

[26] Gromov M., Lawson Jr. H.B., Positive scalar curvature and the Dirac operator on complete Riemannian manifolds, Inst. Hautes Études Sci. Publ. Math. 58 (1983), 83-196.

[27] Joyce D., A new construction of compact 8-manifolds with holonomy Spin(7), J. Differential Geom. 53 (1999), 89-130, arXiv:math.DG/9910002.

[28] Kasparov G.G., Equivariant KK-theory and the Novikov conjecture, Invent. Math. 91 (1988), $147-201$.

[29] Kasparov G.G., K-theory, group $C^{*}$-algebras, and higher signatures (conspectus), in Novikov Conjectures, Index Theorems and Rigidity, Vol. 1 (Oberwolfach, 1993), London Math. Soc. Lecture Note Ser., Vol. 226, Cambridge University Press, Cambridge, 1995, 101-146.

[30] Kreck M., Stolz S., Nonconnected moduli spaces of positive sectional curvature metrics, J. Amer. Math. Soc. 6 (1993), 825-850.

[31] Lawson Jr. H.B., Michelsohn M.-L., Spin geometry, Princeton Mathematical Series, Vol. 38, Princeton University Press, Princeton, NJ, 1989.

[32] Leichtnam E., Piazza P., Spectral sections and higher Atiyah-Patodi-Singer index theory on Galois coverings, Geom. Funct. Anal. 8 (1998), 17-58.

[33] Leichtnam E., Piazza P., On higher eta-invariants and metrics of positive scalar curvature, K-Theory 24 (2001), 341-359.

[34] Leichtnam E., Piazza P., Dirac index classes and the noncommutative spectral flow, J. Funct. Anal. 200 (2003), 348-400.

[35] Mazzeo R., Elliptic theory of differential edge operators. I, Comm. Partial Differential Equations 16 (1991), $1615-1664$.

[36] Piazza P., Schick T., Bordism, rho-invariants and the Baum-Connes conjecture, J. Noncommut. Geom. 1 (2007), 27-111, arXiv:math.KT/0407388.

[37] Piazza P., Schick T., Groups with torsion, bordism and rho invariants, Pacific J. Math. 232 (2007), 355-378, arXiv:math.GN/0604319.

[38] Piazza P., Schick T., Rho-classes, index theory and Stolz' positive scalar curvature sequence, J. Topol. 7 (2014), 965-1004, arXiv:1210.6892.

[39] Piazza P., Vertman B., Eta and rho invariants on manifolds with edges, Ann. Inst. Fourier (Grenoble) 69 (2019), 1955-2035, arXiv:1604.07420.

[40] Piazza P., Zenobi V.F., Singular spaces, groupoids and metrics of positive scalar curvature, J. Geom. Phys. 137 (2019), 87-123, arXiv:1803.02697.

[41] Rosenberg J., $C^{*}$-algebras, positive scalar curvature and the Novikov conjecture. II, in Geometric Methods in Operator Algebras (Kyoto, 1983), Pitman Res. Notes Math. Ser., Vol. 123, Longman Sci. Tech., Harlow, 1986, 341-374.

[42] Rosenberg J., $C^{*}$-algebras, positive scalar curvature, and the Novikov conjecture. III, Topology 25 (1986), 319-336.

[43] Schick T., A counterexample to the (unstable) Gromov-Lawson-Rosenberg conjecture, Topology 37 (1998), 1165-1168, arXiv:math.GT/0403063.

[44] Stolz S., Concordance classes of positive scalar curvature metrics, available at https://www3.nd.edu/ stolz/preprint.html.

[45] Stolz S., Positive scalar curvature metrics - existence and classification questions, in Proceedings of the International Congress of Mathematicians, Vols. 1, 2 (Zürich, 1994), Birkhäuser, Basel, 1995, 625-636.

[46] Stolz S., Manifolds of positive scalar curvature, in Topology of High-Dimensional Manifolds, No. 1, 2 (Trieste, 2001), ICTP Lect. Notes, Vol. 9, Abdus Salam Int. Cent. Theoret. Phys., Trieste, 2002, 661-709.

[47] Wahl C., The Atiyah-Patodi-Singer index theorem for Dirac operators over $C^{*}$-algebras, Asian J. Math. 17 (2013), 265-319, arXiv:0901.0381.

[48] Xie Z., Yu G., A relative higher index theorem, diffeomorphisms and positive scalar curvature, Adv. Math. 250 (2014), 35-73, arXiv:1204.3664.

[49] Xie Z., Yu G., Zeidler R., On the range of the relative higher index and the higher rho-invariant for positive scalar curvature, arXiv:1712.03722. 